\title{
A Theoretical Study of the Antioxidant Activity of Natural Depsidones
}

Mai Van Bay ${ }^{1}$, Pham Cam Nam, ${ }^{2}$ Duong Tuan Quang, ${ }^{3}$ Adam Mechler ${ }^{4}$, Nguyen Khoa Hien ${ }^{5}$, Nguyen Thi Hoa ${ }^{6}$ and Quan V. Vo ${ }^{7 *}$

${ }^{1}$ Department of Chemistry, The University of Da Nang, University of Science and Education, Da Nang 550000, Vietnam.

${ }^{2}$ Department of Chemical Engineering, The University of Da Nang, University of Science and Technology, Da Nang 550000, Vietnam

${ }^{3}$ University of Education, Hue University, Hue City 530000, Vietnam

${ }^{4}$ Department of Chemistry and Physics, La Trobe University, Victoria 3086, Australia

${ }^{5}$ Mientrung Institute for Scientific Research, Vietnam Academy of Science and Technology, Hue City 530000, Vietnam

${ }^{6}$ Academic Affairs, The University of Danang-University of Technology and Education, Da Nang 550000, Vietnam.

${ }^{7}$ Institute of Research and Development, Duy Tan University, Danang 550000, Vietnam.

*Corresponding author: vovanquan2@duytan.edu.vn

\section{Table of Contents}

Table S1: BDE values of the $\mathrm{X}-\mathrm{H}(\mathrm{X}=\mathrm{O}, \mathrm{C})$ bond breaking of different compounds in the gas phase using B3LYP/3-21G and ROB3LYP/6-311G++G(2df,2p)//B3LYP/6-311G(d,p) methods.

Table S2: Proton affinities (PA) of the $\mathrm{X}-\mathrm{H}(\mathrm{X}=\mathrm{O}, \mathrm{C})$ bond breaking in the gas phase calculated using B3LYP/3$21 \mathrm{G}$ and ROB3LYP/6-311G++G(2df,2p)//B3LYP/6-311G(d,p) methods. . 56

Table S3: Cartesian coordinates and molecular enthalpies of all parent molecules, radicals and anions optimized at B3LYP/6-311G $(d, p)$ level of theory in the gas phase. S10

Table S4: Cartesian coordinates of the TS of the selective compounds and $\mathrm{CH}_{3} \mathrm{OO}$. following the HAT mechanism optimized at M05-2X/6-311++G(d,p) level of theory in the gas phase S50

Table S5. The calculated rate constants $\left(k_{\mathrm{app}}, \mathrm{M}^{-1} \mathrm{~s}^{-1}, 298.15 \mathrm{~K}\right)$ of the reaction between the depsidones and the radicals in water following SET mechanism at the M05-2X/6-31+G(d) method. S58

Table S6. The method to compute rate constant following the transition state theory and the quantum mechanics based test for overall free radical scavenging activity (QM-ORSA) protocol... S59

Figure S1: IRC plots for all transition states related to the reaction of $\mathrm{CH}_{3} \mathrm{OO}$ radical with the selective depsidones S60 
Table S7. The lowest calculated BDEs, IEs and PAs of the depsidones by the (RO)B3LYP-GD3/6$311++G(2 d f, 2 p) / / B 3 L Y P-G D 3 / 6-311 G(d, p)$ level and error bars compared with the the (RO)B3LYP/6$311++G(2 d f, 2 p) / / B 3 L Y P / 6-311 G(d, p)$ level in the gas phase......................................................... S61

Table S8. Calculated $\Delta \mathrm{G}^{\neq}(\mathrm{kcal} / \mathrm{mol}), \kappa$, and $k_{\text {eck }}\left(\mathrm{M}^{-1} \mathrm{~s}^{-1}\right)$ at $298.15 \mathrm{~K}$ of the reaction between the selective depsidones and $\mathrm{CH}_{3} \mathrm{OO} 0^{\circ}$ in the gas phase by using the M05-2X-GD3/6-311++G(d,p) method......... S62

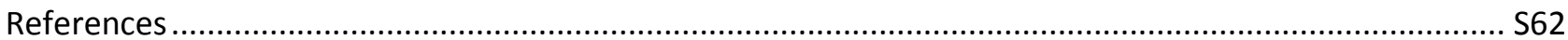


Table S1: $B D E$ values of the $X-H \quad(X=0, \quad C)$ bond breaking of different compounds in the gas phase using B3LYP/3-21G and ROB3LYP/6-311G++G(2df,2p)//B3LYP/6-311G(d,p) methods.

\begin{tabular}{|c|c|c|}
\hline Compounds & B3LYP/3-21G & $\begin{array}{c}\text { (RO)B3LYP/6- } \\
311++G(2 d f, 2 p) / / B 3 L Y P / 6- \\
311 G(d, p) \\
\end{array}$ \\
\hline \multicolumn{3}{|l|}{1} \\
\hline $1-\mathrm{C} 1-\mathrm{H}$ & 78.8 & 79.5 \\
\hline $1-\mathrm{C5}^{\prime}-\mathrm{H}$ & 70.9 & 76.9 \\
\hline 1-C9-H & 115.9 & \\
\hline 1-CH2OH-5-H & 96.8 & \\
\hline 1-CH3-8-H & 90.1 & \\
\hline 1-CHO-11-H & 99.3 & \\
\hline $1-\mathrm{OH}-1-\mathrm{H}$ & 97.7 & \\
\hline $1-\mathrm{OH}-10-\mathrm{H}$ & 102.7 & \\
\hline \multicolumn{3}{|l|}{2} \\
\hline 2-C1-H & 79.2 & 79.9 \\
\hline 2-C9-H & 115.9 & \\
\hline $2-\mathrm{CH} 3-5-\mathrm{H}$ & 88.8 & 88.3 \\
\hline 2-CH3-8-H & 90.1 & \\
\hline 2-CHO-11-H & 99.2 & \\
\hline $2-\mathrm{OH}-1-\mathrm{H}$ & 97.5 & \\
\hline $2-\mathrm{OH}-4-\mathrm{H}$ & 90.4 & \\
\hline $2-\mathrm{OH}-10-\mathrm{H}$ & 101.9 & \\
\hline \multicolumn{3}{|l|}{3} \\
\hline 3-C1-H & 79.5 & 80.4 \\
\hline $3-\mathrm{CH} 3-5-\mathrm{H}$ & 88.9 & 88.3 \\
\hline $3-\mathrm{CH} 3-8-\mathrm{H}$ & 90.2 & \\
\hline 3-CHO-11-H & 91.9 & \\
\hline $3-\mathrm{OCH} 3-10-\mathrm{H}$ & 98.7 & \\
\hline $3-\mathrm{OH}-1-\mathrm{H}$ & 96.9 & \\
\hline $3-\mathrm{OH}-4-\mathrm{H}$ & 90.8 & \\
\hline \multicolumn{3}{|l|}{4} \\
\hline 4-C1-H & 77.1 & 79.9 \\
\hline $4-\mathrm{CH} 2-11-\mathrm{H}$ & 81.6 & 81.7 \\
\hline $4-\mathrm{CH} 2 \mathrm{OH}-12-\mathrm{H}$ & 92.6 & \\
\hline $4-\mathrm{CH} 3-5-\mathrm{H}$ & 90.2 & \\
\hline $4-\mathrm{CH} 3-8-\mathrm{H}$ & 90.7 & \\
\hline $4-\mathrm{OH}-1-\mathrm{H}$ & 97.3 & \\
\hline $4-\mathrm{OH}-10-\mathrm{H}$ & 89.4 & \\
\hline \multicolumn{3}{|l|}{5} \\
\hline $5-\mathrm{C} 11^{\prime}-\mathrm{H}$ & 78.1 & 74.4 \\
\hline $5-\mathrm{CH} 2 \mathrm{OH}-11-\mathrm{H}$ & 97.4 & \\
\hline
\end{tabular}




\begin{tabular}{|c|c|c|}
\hline $5-\mathrm{CH} 3-5-\mathrm{H}$ & 88.8 & \\
\hline 5-CH3-8-H & 89.9 & \\
\hline $5-\mathrm{OCH} 3-10-\mathrm{H}$ & 98.1 & \\
\hline $5-\mathrm{OH}-1-\mathrm{H}$ & 93.9 & \\
\hline $5-\mathrm{OH}-4-\mathrm{H}$ & 90.6 & \\
\hline \multicolumn{3}{|l|}{6} \\
\hline 6-C1-H & 76.3 & 75.2 \\
\hline $6-\mathrm{CH} 3-5-\mathrm{H}$ & 88.8 & 88.2 \\
\hline $6-\mathrm{CH} 3-8-\mathrm{H}$ & 90.6 & \\
\hline 6-COOH-11-H & 100.5 & \\
\hline $6-\mathrm{OH}-1-\mathrm{H}$ & 94.9 & \\
\hline $6-\mathrm{OH}-4-\mathrm{H}$ & 90.5 & \\
\hline \multicolumn{3}{|l|}{7} \\
\hline $7-\mathrm{C} 1-\mathrm{H}$ & 84.5 & 83.6 \\
\hline 7-CH3-9-H & 90.8 & \\
\hline $7-\mathrm{OH}-4-\mathrm{H}$ & 91.5 & \\
\hline 7-OH-7-H & 85.4 & \\
\hline \multicolumn{3}{|l|}{8} \\
\hline $8-\mathrm{C} 7-\mathrm{H}$ & 115.5 & \\
\hline 8-CH3-1-H & 88.1 & 87.7 \\
\hline $8-\mathrm{CH} 3-4-\mathrm{H}$ & 89.2 & 88.3 \\
\hline $8-\mathrm{CH} 3-6-\mathrm{H}$ & 90.2 & \\
\hline 8-CH3-9-H & 90.2 & 88.7 \\
\hline $8-\mathrm{COOH}-2-\mathrm{H}$ & 82.9 & \\
\hline $8-\mathrm{OH}-3-\mathrm{H}$ & 95.2 & \\
\hline $8-\mathrm{OH}-8-\mathrm{H}$ & 80.5 & 85.8 \\
\hline \multicolumn{3}{|l|}{9} \\
\hline 9-C9'-H & 71.8 & 82.4 \\
\hline 9-CH2OH-9-H & 96.5 & \\
\hline 9-CH3-1-H & 89.6 & \\
\hline 9-CH3-6-H & 88.3 & \\
\hline 9-CHO-4-H & 93.9 & \\
\hline 9-COOH-7-H & 101.7 & \\
\hline $9-\mathrm{OH}-3-\mathrm{H}$ & 97.7 & \\
\hline 9-OH-8-H & 95.3 & \\
\hline \multicolumn{3}{|l|}{10} \\
\hline $10-\mathrm{Cg}^{\prime}-\mathrm{H}$ & 72.0 & 78.3 \\
\hline $10-\mathrm{CH} 2 \mathrm{OH}-9-\mathrm{H}$ & 96.6 & \\
\hline 10-CH3-1-H & 90.1 & \\
\hline 10-CH3-4-H & 89.9 & \\
\hline $10-\mathrm{CH} 3-6-\mathrm{H}$ & 88.3 & \\
\hline
\end{tabular}




\begin{tabular}{|c|c|c|}
\hline 10-COOH-7-H & 101.1 & \\
\hline $10-\mathrm{OH}-3-\mathrm{H}$ & 81.7 & \\
\hline $10-\mathrm{OH}-8-\mathrm{H}$ & 94.5 & \\
\hline \multicolumn{3}{|l|}{11} \\
\hline 11-CH3-1-H & 90.8 & 89.4 \\
\hline 11-CH3-6-H & 90.2 & 89.9 \\
\hline 11-COOCH3-7-H & 97.4 & 97.3 \\
\hline 11-OCH3-8-H & 97.3 & 96.3 \\
\hline $11-\mathrm{O} 8-\mathrm{H}$ & 84.8 & 87.7 \\
\hline \multicolumn{3}{|l|}{12} \\
\hline 12-CH2-1-H & 82.4 & \\
\hline 12-CH2-6-H & 90.9 & \\
\hline $12-\mathrm{CH} 2 \mathrm{CH} 2-6-\mathrm{H}$ & 99.5 & \\
\hline $12-\mathrm{CH} 2 \mathrm{CH} 2 \mathrm{CH} 2-6-\mathrm{H}$ & 98.1 & \\
\hline $12-\mathrm{CH} 2 \mathrm{CH} 2 \mathrm{CH} 2 \mathrm{CH} 2-6-\mathrm{H}$ & 98.7 & \\
\hline $12-\mathrm{CH} 2 \mathrm{CH} 2 \mathrm{CH} 2 \mathrm{CH} 2 \mathrm{CH} 3-6-\mathrm{H}$ & 101.9 & \\
\hline $12-\mathrm{CH} 2 \mathrm{COCH} 2-1-\mathrm{H}$ & 91.7 & \\
\hline $12-\mathrm{CH} 2 \mathrm{COCH} 2 \mathrm{CH} 2-1-\mathrm{H}$ & 95.8 & \\
\hline $12-\mathrm{CH} 2 \mathrm{COCH} 2 \mathrm{CH} 2 \mathrm{CH} 2-1-\mathrm{H}$ & 97.2 & \\
\hline $12-\mathrm{CH} 2 \mathrm{COCH} 2 \mathrm{CH} 2 \mathrm{CH} 2 \mathrm{CH} 2-1-\mathrm{H}$ & 95.8 & \\
\hline $12-\mathrm{CH} 2 \mathrm{COCH} 2 \mathrm{CH} 2 \mathrm{CH} 2 \mathrm{CH} 2 \mathrm{CH} 3-1-\mathrm{H}$ & 101.2 & \\
\hline $12-\mathrm{COO}-\mathrm{H}$ & 84.2 & \\
\hline $12-\mathrm{O} 3-\mathrm{H}$ & 83.8 & 88.2 \\
\hline $12-08-\mathrm{H}$ & 94.5 & 84.5 \\
\hline
\end{tabular}


Table S2: Proton affinities (PA) of the $X-H(X=0, C)$ bond breaking in the gas phase calculated using B3LYP/3-21G and ROB3LYP/6-311G++G(2df,2p)//B3LYP/6-311G(d,p) methods.

\begin{tabular}{|c|c|c|}
\hline Compounds & B3lyp/3-21g & $\begin{array}{l}\text { (RO)B3LYP/6-311++G(2df,2p)//B3LYP/6- } \\
\text { 311G(d,p) }\end{array}$ \\
\hline \multicolumn{3}{|c|}{1} \\
\hline 1-C1-H & 345.2 & \\
\hline 1-C5-CH2-H & 350.9 & \\
\hline 1-C9-H & 382.3 & \\
\hline 1-CH2OH-5-H & 375.4 & \\
\hline 1-CH3-8-H & 343.3 & \\
\hline 1-CHO-11-H & 368 & \\
\hline 1-OH-1-H & 311.2 & 310.1 \\
\hline $1-\mathrm{OH}-4-\mathrm{H}$ & 327.4 & \\
\hline 1-OH-10-H & 335 & \\
\hline \multicolumn{3}{|c|}{2} \\
\hline 2-C1-H & 349.9 & \\
\hline 2-C9-H & 386.4 & \\
\hline $2-\mathrm{CH} 3-5-\mathrm{H}$ & 361.4 & \\
\hline $2-\mathrm{CH} 3-8-\mathrm{H}$ & 347.5 & \\
\hline 2-CHO-11-H & 371.3 & \\
\hline 2-OH-1-H & 314.2 & 312.2 \\
\hline $2-\mathrm{OH}-4-\mathrm{H}$ & 331.1 & \\
\hline $2-\mathrm{OH}-10-\mathrm{H}$ & 339.1 & \\
\hline \multicolumn{3}{|c|}{3} \\
\hline $3-\mathrm{C} 1-\mathrm{H}$ & 351.6 & \\
\hline $3-\mathrm{CH} 3-5-\mathrm{H}$ & 362.6 & \\
\hline $3-\mathrm{CH} 3-8-\mathrm{H}$ & 346.5 & \\
\hline 3-CHO-11-H & 375.6 & \\
\hline 3-OCH3-10-H & 383.1 & \\
\hline $3-\mathrm{OH}-1-\mathrm{H}$ & 317.3 & 314.6 \\
\hline $3-\mathrm{OH}-4-\mathrm{H}$ & 332.6 & \\
\hline \multicolumn{3}{|c|}{4} \\
\hline $4-\mathrm{C} 1-\mathrm{H}$ & 346.1 & \\
\hline 4-CH2-11-H & 367.3 & \\
\hline $4-\mathrm{CH} 2 \mathrm{OH}-12-\mathrm{H}$ & 322.5 & \\
\hline $4-\mathrm{CH} 3-5-\mathrm{H}$ & 362.3 & \\
\hline 4-CH3-8-H & 357.7 & \\
\hline 4-OH-1-H & 315.7 & 316.4 \\
\hline $4-\mathrm{OH}-10-\mathrm{H}$ & 331.3 & \\
\hline \multicolumn{3}{|c|}{5} \\
\hline $5-\mathrm{C} 1-\mathrm{H}$ & 362.2 & \\
\hline 5-CH2-11-H & 376 & \\
\hline $5-\mathrm{CH} 2 \mathrm{OH}-11-\mathrm{H}$ & 360 & \\
\hline 5-CH3-5-H & 370.5 & \\
\hline
\end{tabular}




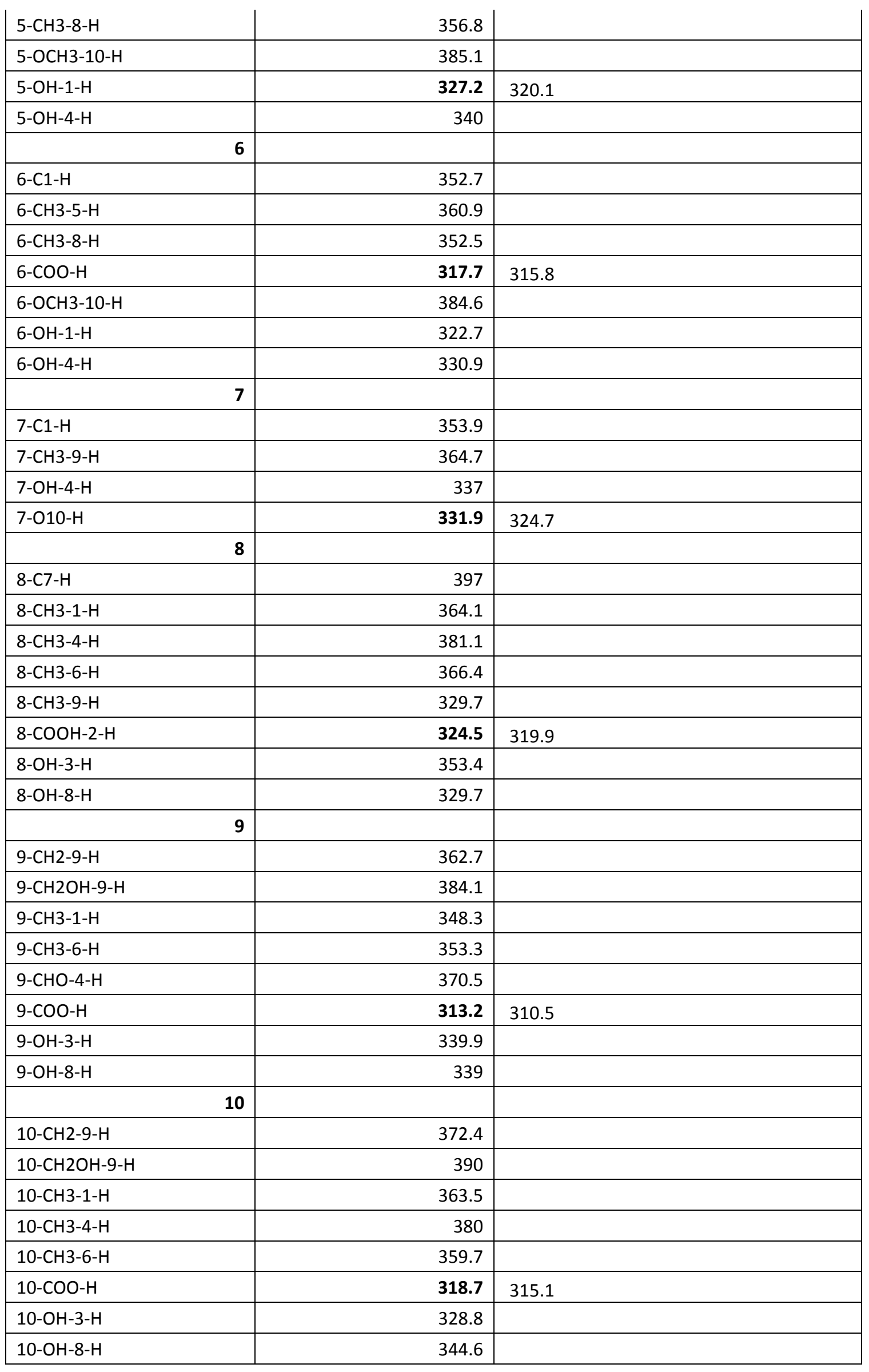




\begin{tabular}{|c|c|c|}
\hline 11 & & \\
\hline 11-CH3-1-H & 353.7 & \\
\hline $11-\mathrm{CH} 3-6-\mathrm{H}$ & 366.5 & \\
\hline 11-COOCH3-7-H & 398.9 & \\
\hline 11-OCH3-8-H & 395.9 & \\
\hline 11-OH-3-H & 319.7 & 320.6 \\
\hline 12 & & \\
\hline $12-\mathrm{CH} 2-1-\mathrm{H}$ & 340.9 & \\
\hline $12-\mathrm{CH} 2-6-\mathrm{H}$ & 367.4 & \\
\hline $12-\mathrm{CH} 2 \mathrm{CH} 2-6-\mathrm{H}$ & 373.4 & \\
\hline $12-\mathrm{CH} 2 \mathrm{CH} 2 \mathrm{CH} 2-6-\mathrm{H}$ & 327.7 & \\
\hline $12-\mathrm{CH} 2 \mathrm{CH} 2 \mathrm{CH} 2 \mathrm{CH} 2-6-\mathrm{H}$ & 328.6 & \\
\hline $12-\mathrm{CH} 2 \mathrm{CH} 2 \mathrm{CH} 2 \mathrm{CH} 2 \mathrm{CH} 3-6-\mathrm{H}$ & 326.7 & \\
\hline $12-\mathrm{CH} 2 \mathrm{COCH} 2-1-\mathrm{H}$ & 371.9 & \\
\hline $12-\mathrm{CH} 2 \mathrm{COCH} 2 \mathrm{CH} 2-1-\mathrm{H}$ & 396.0 & \\
\hline $12-\mathrm{CH} 2 \mathrm{COCH} 2 \mathrm{CH} 2 \mathrm{CH} 2-1-\mathrm{H}$ & 346.4 & \\
\hline $\begin{array}{l}12-\mathrm{CH} 2 \mathrm{COCH} 2 \mathrm{CH} 2 \mathrm{CH} 2 \mathrm{CH} 2- \\
1-\mathrm{H}\end{array}$ & 345.2 & \\
\hline $\begin{array}{l}12- \\
\mathrm{CH} 2 \mathrm{COCH} 2 \mathrm{CH} 2 \mathrm{CH}_{2} \mathrm{CH} 2 \mathrm{CH} 3- \\
1-\mathrm{H}\end{array}$ & 344.5 & \\
\hline $12-\mathrm{COOH}-7-\mathrm{H}$ & 322.5 & \\
\hline $12-\mathrm{OH}-3-\mathrm{H}$ & 325.3 & \\
\hline $12-\mathrm{O} 8-\mathrm{H}$ & 318.5 & 316.4 \\
\hline
\end{tabular}


Table S3: Cartesian coordinates and molecular enthalpies of all parent molecules, radicals and anions optimized at $B 3 L Y P / 6-311 G(d, p)$ level of theory in the gas phase.

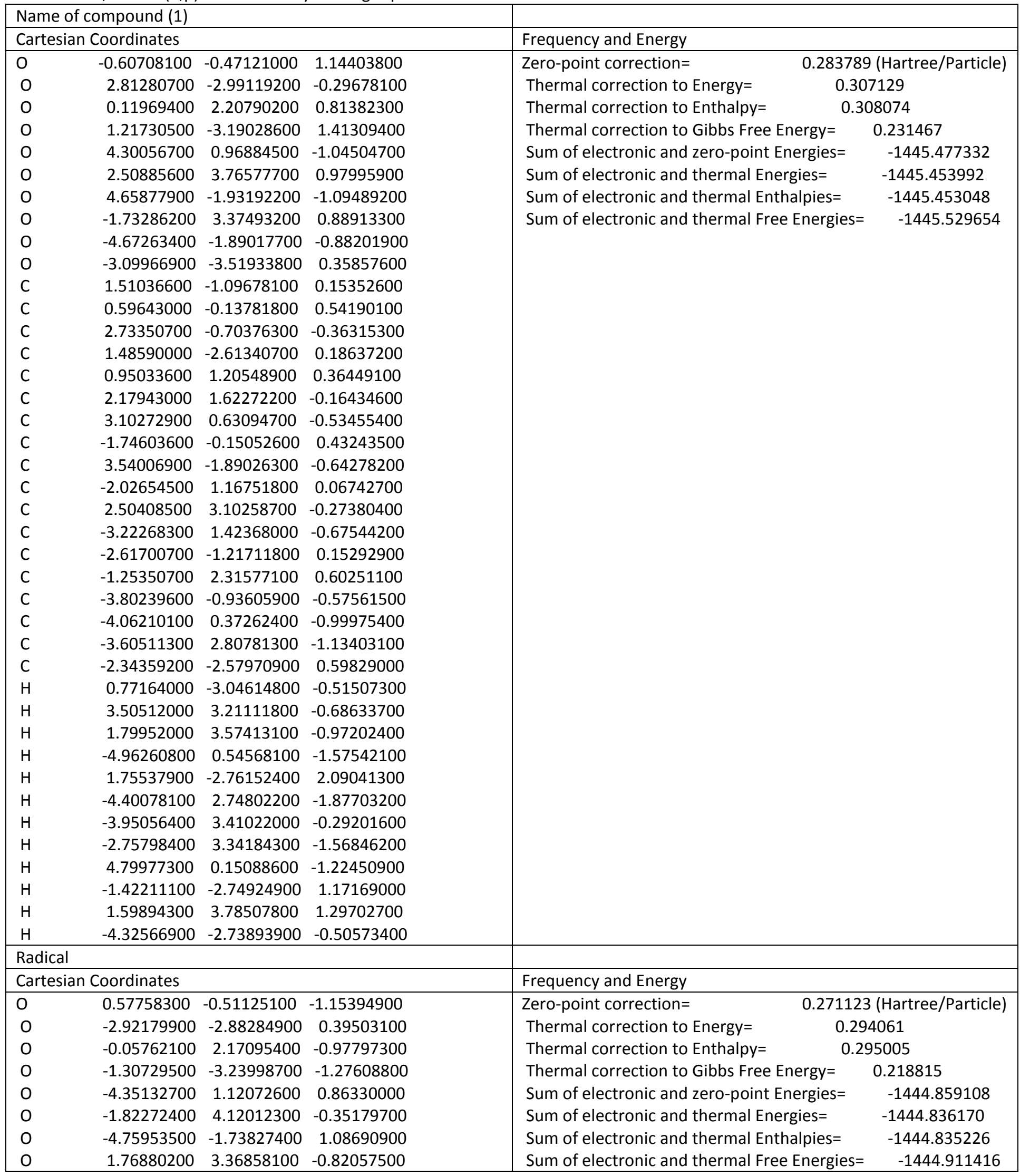




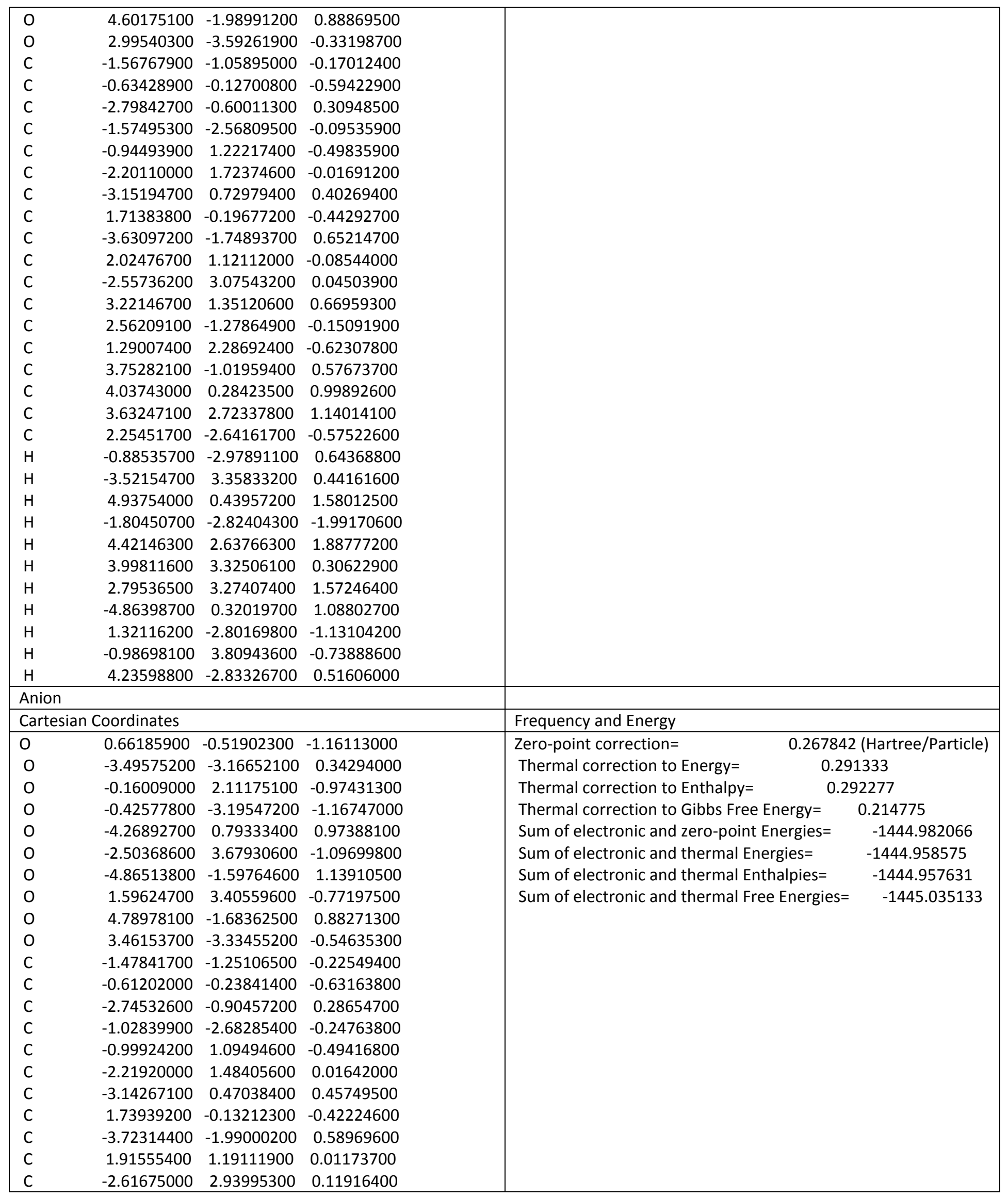




\begin{tabular}{|c|c|c|c|c|c|}
\hline \multirow{2}{*}{\multicolumn{3}{|c|}{$304365600 \quad 149369400 \quad 083097000$}} & & & \\
\hline & 3.04365600 & 1.49369400 & 0.83097000 & & \\
\hline C & 2.71275300 & -1.12573600 & -0.17709400 & & \\
\hline $\mathrm{C}$ & 1.12880400 & 2.31170600 & -0.57099000 & & \\
\hline C & 3.83987300 & -0.78766200 & 0.61234000 & & \\
\hline C & 3.95838800 & 0.50126300 & 1.14100600 & & \\
\hline $\mathrm{C}$ & 3.28315800 & 2.87352300 & 1.39419800 & & \\
\hline $\mathrm{C}$ & 2.59845400 & -2.46989400 & -0.73252000 & & \\
\hline $\mathrm{H}$ & -1.21590500 & -3.23608600 & 0.68492100 & & \\
\hline $\mathrm{H}$ & -3.66447100 & 2.96783300 & 0.41320100 & & \\
\hline $\mathrm{H}$ & -2.03310900 & 3.42648100 & 0.91747100 & & \\
\hline $\mathrm{H}$ & 4.80995300 & 0.71327600 & 1.77591400 & & \\
\hline $\mathrm{H}$ & 4.01973100 & 2.82324800 & 2.19830300 & & \\
\hline $\mathrm{H}$ & 3.64581000 & 3.54911100 & 0.61795200 & & \\
\hline $\mathrm{H}$ & 2.36523500 & 3.31738000 & 1.78346300 & & \\
\hline $\mathrm{H}$ & -4.82543000 & -0.56926700 & 1.18597100 & & \\
\hline $\mathrm{H}$ & 1.71003100 & -2.69533100 & -1.33124500 & & \\
\hline $\mathrm{H}$ & -1.58297400 & 3.61020200 & -1.37248500 & & \\
\hline $\mathrm{H}$ & 4.52153700 & -2.52196400 & 0.41662400 & & \\
\hline \multicolumn{6}{|c|}{ Radical cation } \\
\hline \multicolumn{4}{|c|}{ Cartesian Coordinates } & \multicolumn{2}{|l|}{ Frequency and Energy } \\
\hline 0 & -0.55617200 & -0.77175100 & -0.31835700 & Zero-point correction= & (Hartree/Particle) \\
\hline $\mathrm{O}$ & 3.23987500 & -2.89995900 & 0.30834100 & Thermal correction to Energy= & 205 \\
\hline $\mathrm{O}$ & -0.02618200 & 1.96265500 & -0.82454300 & Thermal correction to Enthalpy= & 6149 \\
\hline $\mathrm{O}$ & 1.05259900 & -3.18225100 & 1.11216600 & Thermal correction to Gibbs Free Energy= & 0.227179 \\
\hline $\mathrm{O}$ & 4.57633900 & 1.13133400 & -0.16346400 & Sum of electronic and zero-point Energies= & -1445.177822 \\
\hline $\mathrm{O}$ & 1.95435100 & 3.79984700 & 0.54737800 & Sum of electronic and thermal Energies= & -1445.153932 \\
\hline $\mathrm{O}$ & 5.15236500 & -1.65726200 & 0.30055800 & Sum of electronic and thermal Enthalpies= & -1445.152988 \\
\hline $\mathrm{O}$ & -1.17000400 & 3.23728600 & 0.60769600 & Sum of electronic and thermal Free Energies= & -1445.231958 \\
\hline $\mathrm{O}$ & -5.14772100 & -1.72787400 & -0.12366700 & & \\
\hline 0 & -3.35551900 & -3.52551900 & -0.51488000 & & \\
\hline $\mathrm{C}$ & 1.74652200 & -1.14656200 & -0.09104900 & & \\
\hline $\mathrm{C}$ & 0.67260700 & -0.25197800 & -0.27607700 & & \\
\hline $\mathrm{C}$ & 3.01363200 & -0.65569700 & -0.06085300 & & \\
\hline $\mathrm{C}$ & 1.81533600 & -2.65921300 & 0.09675300 & & \\
\hline $\mathrm{C}$ & 0.97966800 & 1.12389400 & -0.48543700 & & \\
\hline $\mathrm{C}$ & 2.27239500 & 1.63263500 & -0.45412600 & & \\
\hline $\mathrm{C}$ & 3.33042300 & 0.71707300 & -0.22374000 & & \\
\hline $\mathrm{C}$ & -1.81852400 & -0.24342900 & -0.18260400 & & \\
\hline $\mathrm{C}$ & 3.95893500 & -1.75619900 & 0.19837700 & & \\
\hline C & -2.12758100 & 1.11172300 & 0.07152800 & & \\
\hline $\mathrm{C}$ & 2.49915600 & 3.13342900 & -0.57453500 & & \\
\hline $\mathrm{C}$ & -3.49258500 & 1.45750600 & 0.31797900 & & \\
\hline $\mathrm{C}$ & -2.80158300 & -1.25136700 & -0.23489900 & & \\
\hline $\mathrm{C}$ & -1.12355500 & 2.17989500 & 0.06283800 & & \\
\hline $\mathrm{C}$ & -4.15469400 & -0.86896700 & -0.05773900 & & \\
\hline $\mathrm{C}$ & -4.46559500 & 0.47296100 & 0.23008600 & & \\
\hline $\mathrm{C}$ & -3.93149300 & 2.86114100 & 0.63628700 & & \\
\hline $\mathrm{C}$ & -2.48260400 & -2.67663900 & -0.41878700 & & \\
\hline $\mathrm{H}$ & 1.53634300 & -3.19383200 & -0.81283100 & & \\
\hline $\mathrm{H}$ & 3.56993000 & 3.33102700 & -0.58274600 & & \\
\hline $\mathrm{H}$ & 2.07913200 & 3.48939800 & -1.52018900 & & \\
\hline $\mathrm{H}$ & -5.50861600 & 0.71885400 & 0.38433500 & & \\
\hline $\mathrm{H}$ & 1.33857800 & -2.82718400 & 1.96459100 & & \\
\hline $\mathrm{H}$ & -5.01812100 & 2.90816200 & 0.68711600 & & \\
\hline
\end{tabular}




\begin{tabular}{|c|c|c|c|c|c|}
\hline $\mathrm{H}$ & -3.51869100 & 3.19390800 & 1.59077300 & & \\
\hline $\mathrm{H}$ & -3.58662000 & 3.57449400 & -0.11534300 & & \\
\hline $\mathrm{H}$ & 5.17866800 & 0.37302800 & 0.00799400 & & \\
\hline $\mathrm{H}$ & -1.42605000 & -2.96847500 & -0.44790200 & & \\
\hline $\mathrm{H}$ & 1.02822700 & 4.01490900 & 0.38245800 & & \\
\hline $\mathrm{H}$ & -4.77498100 & -2.62759200 & -0.31210400 & & \\
\hline & ompound (2) & & & & \\
\hline Car & Coordinates & & & Frequency and Energy & \\
\hline 0 & -0.48790800 & -0.41824800 & 1.17545100 & Zero-point correction $=$ & (Hartree/Particle) \\
\hline $\mathrm{O}$ & 3.17577700 & -2.50273800 & -0.35530700 & Thermal correction to Energy= & 0564 \\
\hline 0 & -0.04320400 & 2.32370200 & 1.00912300 & Thermal correction to Enthalpy= & 1509 \\
\hline 0 & 1.59404100 & -2.96127700 & 1.31750800 & Thermal correction to Gibbs Free Energy= & 0.226229 \\
\hline 0 & 4.26055200 & 1.62944700 & -0.85732300 & Sum of electronic and zero-point Energies= & -1370.252725 \\
\hline 0 & 4.91519000 & -1.22242300 & -1.06397300 & Sum of electronic and thermal Energies= & -1370.230099 \\
\hline $\mathrm{O}$ & -2.00774800 & 3.29423400 & 1.06736000 & Sum of electronic and thermal Enthalpies= & -1370.229155 \\
\hline $\mathrm{O}$ & -4.35220100 & -2.14912300 & -1.00027200 & Sum of electronic and thermal Free Energies= & -1370.304435 \\
\hline $\mathrm{O}$ & -2.65024400 & -3.66487500 & 0.21292200 & & \\
\hline $\mathrm{C}$ & 1.68799500 & -0.77594400 & 0.18768000 & & \\
\hline $\mathrm{C}$ & 0.68350400 & 0.06680300 & 0.61298100 & & \\
\hline $\mathrm{C}$ & 2.87162900 & -0.23529400 & -0.28942700 & & \\
\hline $\mathrm{C}$ & 1.81294100 & -2.28646000 & 0.13111500 & & \\
\hline $\mathrm{C}$ & 0.89515400 & 1.45161500 & 0.51335200 & & \\
\hline $\mathrm{C}$ & 2.08272700 & 2.01536100 & 0.02292500 & & \\
\hline $\mathrm{C}$ & 3.10001000 & 1.13450300 & -0.38682600 & & \\
\hline $\mathrm{C}$ & -1.64096800 & -0.18356500 & 0.45314900 & & \\
\hline C & 3.79354500 & -1.31716400 & -0.62607100 & & \\
\hline $\mathrm{C}$ & -2.04474700 & 1.11393600 & 0.13566700 & & \\
\hline $\mathrm{C}$ & -3.24294700 & 1.28050300 & -0.62794900 & & \\
\hline $\mathrm{C}$ & 2.29346400 & 3.50232400 & -0.05747600 & & \\
\hline $\mathrm{C}$ & -2.39489300 & -1.31946500 & 0.10968700 & & \\
\hline $\mathrm{C}$ & -1.40631900 & 2.30806100 & 0.74931100 & & \\
\hline $\mathrm{C}$ & -3.58716000 & -1.12665600 & -0.63574700 & & \\
\hline $\mathrm{C}$ & -3.96639600 & 0.16612100 & -1.01541500 & & \\
\hline $\mathrm{C}$ & -3.75167300 & 2.63824000 & -1.04011600 & & \\
\hline C & -1.99594300 & -2.66529600 & 0.50614100 & & \\
\hline $\mathrm{H}$ & 1.15227000 & -2.74586900 & -0.60496700 & & \\
\hline $\mathrm{H}$ & 2.25297000 & 3.84054500 & -1.09791000 & & \\
\hline $\mathrm{H}$ & 1.53549200 & 4.03758800 & 0.51007900 & & \\
\hline $\mathrm{H}$ & 3.28307200 & 3.76454700 & 0.32199600 & & \\
\hline $\mathrm{H}$ & -4.86704800 & 0.27338100 & -1.60672800 & & \\
\hline $\mathrm{H}$ & 2.07230400 & -2.51586200 & 2.02799800 & & \\
\hline $\mathrm{H}$ & -4.52322500 & 2.53229900 & -1.80326900 & & \\
\hline $\mathrm{H}$ & -4.16951600 & 3.16797600 & -0.18240100 & & \\
\hline $\mathrm{H}$ & -2.95260000 & 3.26993900 & -1.43273700 & & \\
\hline $\mathrm{H}$ & 4.84469500 & 0.88077500 & -1.07744000 & & \\
\hline $\mathrm{H}$ & -1.07229300 & -2.76703400 & 1.09190700 & & \\
\hline $\mathrm{H}$ & -3.92969400 & -2.97362200 & -0.64831500 & & \\
\hline & & & & & \\
\hline & Coordinates & & & Frequency and Energy & \\
\hline 0 & 0.48432400 & -0.41663300 & -1.20425500 & Zero-point correction= & (Hartree/Particle) \\
\hline $\mathrm{O}$ & -3.15338600 & -2.54943300 & 0.18955900 & Thermal correction to Energy= & 7146 \\
\hline $\mathrm{O}$ & -0.01703100 & 2.33140500 & -0.98937000 & Thermal correction to Enthalpy= & 38091 \\
\hline 0 & -1.12512900 & -3.19566100 & -0.66283800 & Thermal correction to Gibbs Free Energy= & 0.211965 \\
\hline 0 & -4.32282300 & 1.57379600 & 0.83329600 & Sum of electronic and zero-point Energies= & -1369.627535 \\
\hline
\end{tabular}




\begin{tabular}{|c|c|c|c|c|c|}
\hline 0 & -4.97626900 & -1.36025600 & 0.96949300 & Sum of electronic and thermal Energies= & -1369.604751 \\
\hline $\mathrm{O}$ & 1.93611500 & 3.32373200 & -1.04597500 & Sum of electronic and thermal Enthalpies= & -1369.603807 \\
\hline 0 & 4.37945800 & -2.07382200 & 0.98191400 & Sum of electronic and thermal Free Energies= & -1369.679932 \\
\hline $\mathrm{O}$ & 2.76170200 & -3.60991700 & -0.31347700 & & \\
\hline C & -1.72970400 & -0.82960300 & -0.28507000 & & \\
\hline C & -0.70344400 & 0.05636300 & -0.66726400 & & \\
\hline C & -2.94664000 & -0.28013000 & 0.21253500 & & \\
\hline C & -1.93058300 & -2.20944500 & -0.27799900 & & \\
\hline C & -0.93473000 & 1.41450100 & -0.52977700 & & \\
\hline C & -2.13821000 & 1.97440200 & -0.02701900 & & \\
\hline C & -3.15824600 & 1.09310300 & 0.35074300 & & \\
\hline C & 1.62738100 & -0.16039100 & -0.47087100 & & \\
\hline C & -3.85413800 & -1.33963200 & 0.52446900 & & \\
\hline C & 1.99314900 & 1.14113600 & -0.12405300 & & \\
\hline C & 3.17334100 & 1.32718400 & 0.66155900 & & \\
\hline C & -2.30303100 & 3.46531600 & 0.07219300 & & \\
\hline C & 2.41800100 & -1.27809700 & -0.14499100 & & \\
\hline C & 1.34102600 & 2.33099300 & -0.73372200 & & \\
\hline $\mathrm{C}$ & 3.58939300 & -1.06586700 & 0.62720000 & & \\
\hline C & 3.92292700 & 0.22796700 & 1.04202000 & & \\
\hline C & 3.63476100 & 2.69164100 & 1.10697600 & & \\
\hline C & 2.08384500 & -2.62187300 & -0.59708000 & & \\
\hline $\mathrm{H}$ & -3.30143000 & 3.71235700 & 0.42789100 & & \\
\hline $\mathrm{H}$ & -1.57158100 & 3.89844200 & 0.76082100 & & \\
\hline $\mathrm{H}$ & -2.14287300 & 3.94208100 & -0.89805800 & & \\
\hline $\mathrm{H}$ & 4.80909800 & 0.34974500 & 1.65210200 & & \\
\hline $\mathrm{H}$ & -1.56701100 & -4.04228100 & -0.50987300 & & \\
\hline $\mathrm{H}$ & 4.38979300 & 2.59413800 & 1.88771500 & & \\
\hline $\mathrm{H}$ & 4.05933800 & 3.24552800 & 0.26808200 & & \\
\hline $\mathrm{H}$ & 2.80964100 & 3.29553600 & 1.48926900 & & \\
\hline $\mathrm{H}$ & -4.90976600 & 0.82582000 & 1.03501600 & & \\
\hline $\mathrm{H}$ & 1.19008000 & -2.73111700 & -1.22011900 & & \\
\hline $\mathrm{H}$ & 3.98843400 & -2.90134600 & 0.60128500 & & \\
\hline \multicolumn{6}{|c|}{ Anion } \\
\hline \multicolumn{4}{|c|}{ Cartesian Coordinates } & \multicolumn{2}{|l|}{ Frequency and Energy } \\
\hline 0 & 0.54305600 & -0.48192600 & -1.18521100 & \multicolumn{2}{|c|}{0.262014 (Hartree/Particle) } \\
\hline $\mathrm{O}$ & -3.88468600 & -2.64060600 & 0.37669300 & \multicolumn{2}{|l|}{ Thermal correction to Energy= } \\
\hline $\mathrm{O}$ & -0.02806800 & 2.21378100 & -1.16859800 & \multicolumn{2}{|l|}{ Thermal correction to Enthalpy= } \\
\hline $\mathrm{O}$ & -0.77445000 & -3.03919400 & -1.07051900 & \multicolumn{2}{|c|}{ Thermal correction to Gibbs Free Energy $=0.209876$} \\
\hline 0 & -4.25756000 & 1.40838500 & 0.78983900 & Sum of electronic and zero-point Energies= & -1369.753945 \\
\hline $\mathrm{O}$ & -5.09216000 & -0.89939600 & 1.07007400 & Sum of electronic and thermal Energies= & -1369.731207 \\
\hline $\mathrm{O}$ & 1.84523100 & 3.34266400 & -1.01428800 & Sum of electronic and thermal Enthalpies= & -1369.730263 \\
\hline $\mathrm{O}$ & 4.50542800 & -1.90523400 & 1.02333100 & \multirow[t]{12}{*}{ Sum of electronic and thermal Free Energies= } & \multirow[t]{12}{*}{-1369.806083} \\
\hline $\mathrm{O}$ & 3.06053900 & -3.50583200 & -0.34901800 & & \\
\hline C & -1.66533700 & -0.96147000 & -0.25250600 & & \\
\hline C & -0.70646500 & -0.05512800 & -0.69460500 & & \\
\hline $\mathrm{C}$ & -2.90085800 & -0.47204700 & 0.22703900 & & \\
\hline $\mathrm{C}$ & -1.35373800 & -2.42677000 & -0.19704600 & & \\
\hline $\mathrm{C}$ & -0.95963400 & 1.31997000 & -0.63027700 & & \\
\hline C & -2.13939000 & 1.84158500 & -0.14326600 & & \\
\hline C & -3.15944600 & 0.93867900 & 0.32458200 & & \\
\hline C & 1.63967200 & -0.15626400 & -0.44407200 & & \\
\hline $\mathrm{C}$ & -3.98839800 & -1.43565700 & 0.56219800 & & \\
\hline $\mathrm{C}$ & 1.92999600 & 1.16722200 & -0.08067400 & & \\
\hline
\end{tabular}




\begin{tabular}{|c|c|c|c|c|c|}
\hline \multirow{2}{*}{\multicolumn{3}{|c|}{$\begin{array}{llll}306404800 & 141145500 & 074788200\end{array}$}} & & & \\
\hline & 3.06404800 & 1.41145500 & 0.74788200 & & \\
\hline C & -2.43037500 & 3.31326300 & -0.09568800 & & \\
\hline $\mathrm{C}$ & 2.51111400 & -1.22037300 & -0.11930600 & & \\
\hline C & 1.26173200 & 2.31832500 & -0.75314600 & & \\
\hline C & 3.64825100 & -0.94210700 & 0.67911700 & & \\
\hline $\mathrm{C}$ & 3.87612700 & 0.35872500 & 1.13720200 & & \\
\hline $\mathrm{C}$ & 3.41939400 & 2.79503000 & 1.23560600 & & \\
\hline $\mathrm{C}$ & 2.28574900 & -2.57688200 & -0.60443800 & & \\
\hline $\mathrm{H}$ & -1.62971700 & -2.91742300 & 0.74824700 & & \\
\hline $\mathrm{H}$ & -2.48419400 & 3.66021400 & 0.94245100 & & \\
\hline $\mathrm{H}$ & -1.67584300 & 3.89338600 & -0.62564200 & & \\
\hline $\mathrm{H}$ & -3.41789200 & 3.50477900 & -0.52444400 & & \\
\hline $\mathrm{H}$ & 4.73116000 & 0.52827500 & 1.78028500 & & \\
\hline $\mathrm{H}$ & 4.13566900 & 2.72752800 & 2.05677900 & & \\
\hline $\mathrm{H}$ & 3.85231300 & 3.38750000 & 0.42827800 & & \\
\hline $\mathrm{H}$ & 2.53884000 & 3.34103800 & 1.57883200 & & \\
\hline $\mathrm{H}$ & -4.94799700 & 0.12147800 & 1.06399300 & & \\
\hline $\mathrm{H}$ & 1.39441700 & -2.75320600 & -1.21512100 & & \\
\hline $\mathrm{H}$ & 4.16994300 & -2.74005700 & 0.59631100 & & \\
\hline \multicolumn{6}{|c|}{ Radical cation } \\
\hline \multicolumn{4}{|c|}{ Cartesian Coordinates } & \multicolumn{2}{|l|}{ Frequency and Energy } \\
\hline 0 & -0.44113300 & -0.61315600 & -0.00083800 & Zero-point correction= & (Hartree/Particle) \\
\hline $\mathrm{O}$ & 3.47134100 & -2.45730400 & -0.63765900 & Thermal correction to Energy= & 8951 \\
\hline $\mathrm{O}$ & -0.11138200 & 2.14385100 & 0.65324100 & Thermal correction to Enthalpy= & 9895 \\
\hline $\mathrm{O}$ & 1.44576100 & -3.18167100 & 0.29115600 & Thermal correction to Gibbs Free Energy= & 0.223173 \\
\hline $\mathrm{O}$ & 4.56565600 & 1.57607100 & 0.28366900 & Sum of electronic and zero-point Energies= & -1369.957152 \\
\hline $\mathrm{O}$ & 5.31664300 & -1.14032700 & -0.39156200 & Sum of electronic and thermal Energies= & -1369.934123 \\
\hline $\mathrm{O}$ & -1.31666800 & 3.25966300 & -0.88020500 & Sum of electronic and thermal Enthalpies= & -1369.933179 \\
\hline $\mathrm{O}$ & -4.95417800 & -1.89776200 & 0.22909700 & Sum of electronic and thermal Free Energies= & -1370.009901 \\
\hline $\mathrm{O}$ & -3.01816000 & -3.56631300 & 0.48366800 & & \\
\hline $\mathrm{C}$ & 1.87136800 & -0.81043500 & -0.20965800 & & \\
\hline $\mathrm{C}$ & 0.74521100 & 0.00193500 & 0.02201600 & & \\
\hline $\mathrm{C}$ & 3.11051400 & -0.25904800 & -0.12409300 & & \\
\hline $\mathrm{C}$ & 2.02523500 & -2.28267200 & -0.57752700 & & \\
\hline $\mathrm{C}$ & 0.95759700 & 1.37969300 & 0.34424600 & & \\
\hline $\mathrm{C}$ & 2.22868800 & 1.94649800 & 0.43924900 & & \\
\hline $\mathrm{C}$ & 3.34097600 & 1.09738900 & 0.20779500 & & \\
\hline $\mathrm{C}$ & -1.74595200 & -0.18853700 & 0.02962600 & & \\
\hline $\mathrm{C}$ & 4.12484000 & -1.29311900 & -0.39302300 & & \\
\hline $\mathrm{C}$ & -2.16796400 & 1.14151300 & -0.16628400 & & \\
\hline $\mathrm{C}$ & -3.56381400 & 1.40328100 & -0.28397000 & & \\
\hline $\mathrm{C}$ & 2.42384300 & 3.38930400 & 0.80128700 & & \\
\hline $\mathrm{C}$ & -2.64624900 & -1.26311700 & 0.15957000 & & \\
\hline $\mathrm{C}$ & -1.21772000 & 2.25795200 & -0.25378100 & & \\
\hline $\mathrm{C}$ & -4.03070000 & -0.97126700 & 0.09622600 & & \\
\hline $\mathrm{C}$ & -4.45731200 & 0.35263800 & -0.13459400 & & \\
\hline $\mathrm{C}$ & -4.10520400 & 2.78444200 & -0.53654200 & & \\
\hline $\mathrm{C}$ & -2.21228400 & -2.65639200 & 0.35119200 & & \\
\hline $\mathrm{H}$ & 1.63236300 & -2.50770800 & -1.56977200 & & \\
\hline $\mathrm{H}$ & 3.33141900 & 3.77861300 & 0.34039500 & & \\
\hline $\mathrm{H}$ & 1.56914300 & 3.98601400 & 0.48790700 & & \\
\hline $\mathrm{H}$ & 2.53143900 & 3.50088000 & 1.88536400 & & \\
\hline $\mathrm{H}$ & -5.52428800 & 0.52727300 & -0.19398000 & & \\
\hline $\mathrm{H}$ & 1.85522100 & -3.11208600 & 1.16427500 & & \\
\hline
\end{tabular}




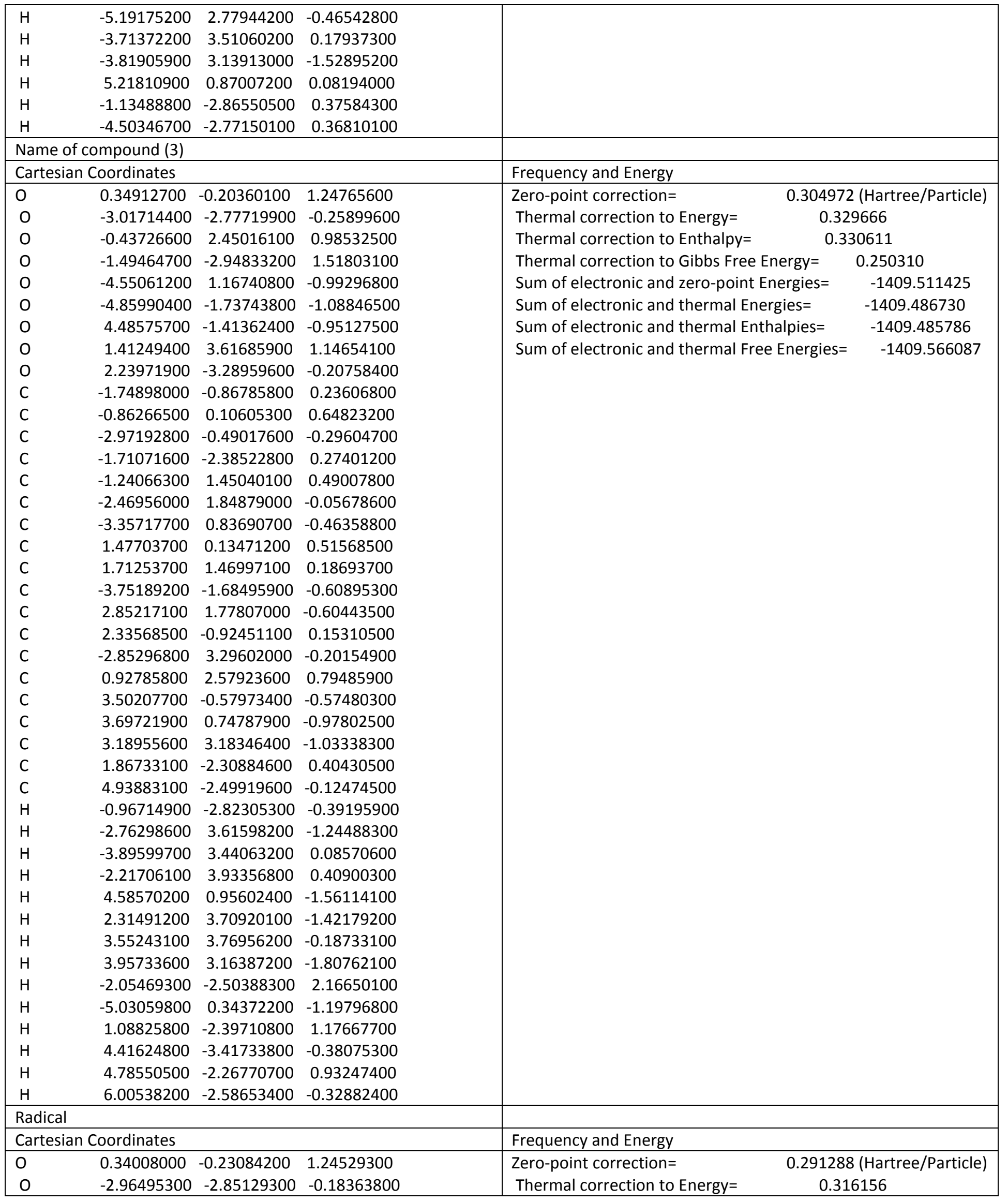




\begin{tabular}{|c|c|c|c|c|c|}
\hline 0 & -0.53459400 & 2.41305200 & 1.01205400 & \multicolumn{2}{|l|}{ Thermal correction to Enthalpy= } \\
\hline 0 & -0.89179000 & -3.21513100 & 0.72548600 & Thermal correction to Gibbs Free Energy= & 0.235951 \\
\hline 0 & -4.66988700 & 1.06798000 & -0.86819100 & Sum of electronic and zero-point Energies= & -1408.885569 \\
\hline 0 & -4.91392000 & -1.91901800 & -1.00472300 & Sum of electronic and thermal Energies= & -1408.860701 \\
\hline 0 & 4.53862800 & -1.25956900 & -0.93293500 & Sum of electronic and thermal Enthalpies= & -1408.859757 \\
\hline 0 & 1.27715900 & 3.64676900 & 1.06350200 & Sum of electronic and thermal Free Energies= & -1408.940907 \\
\hline $\mathrm{O}$ & 2.50879100 & -3.24394000 & 0.04828300 & & \\
\hline $\mathrm{C}$ & -1.79047700 & -0.95484400 & 0.30485400 & & \\
\hline C & -0.89725200 & 0.06474800 & 0.69380900 & & \\
\hline C & -3.06237900 & -0.57605600 & -0.21466600 & & \\
\hline C & -1.80672400 & -2.35002000 & 0.30508000 & & \\
\hline C & -1.31450100 & 1.37706900 & 0.54699000 & & \\
\hline C & -2.57584700 & 1.76753300 & 0.02705100 & & \\
\hline $\mathrm{C}$ & -3.45842900 & 0.75431600 & -0.36374700 & & \\
\hline $\mathrm{C}$ & 1.44864600 & 0.15806100 & 0.50855900 & & \\
\hline $\mathrm{C}$ & 1.60714000 & 1.49430500 & 0.13265900 & & \\
\hline $\mathrm{C}$ & -3.81283800 & -1.74882400 & -0.53777000 & & \\
\hline $\mathrm{C}$ & 2.70859600 & 1.83463100 & -0.69772900 & & \\
\hline $\mathrm{C}$ & 2.38253600 & -0.85392400 & 0.19365300 & & \\
\hline $\mathrm{C}$ & -2.94195300 & 3.22178300 & -0.07842500 & & \\
\hline C & 0.80766600 & 2.58870200 & 0.75148000 & & \\
\hline C & 3.50914200 & -0.46996900 & -0.57472400 & & \\
\hline C & 3.60670500 & 0.84292000 & -1.05085400 & & \\
\hline C & 2.95276500 & 3.23828300 & -1.19223900 & & \\
\hline C & 2.04784800 & -2.24817100 & 0.56887500 & & \\
\hline $\mathrm{C}$ & 5.12553700 & -2.21241700 & -0.03090800 & & \\
\hline $\mathrm{H}$ & -2.27184100 & 3.74980400 & -0.76339300 & & \\
\hline $\mathrm{H}$ & -3.96207600 & 3.32918800 & -0.44226900 & & \\
\hline $\mathrm{H}$ & -2.85617600 & 3.71792200 & 0.89149500 & & \\
\hline $\mathrm{H}$ & 4.46561500 & 1.07839500 & -1.66697100 & & \\
\hline $\mathrm{H}$ & 2.03623900 & 3.70498200 & -1.55851200 & & \\
\hline $\mathrm{H}$ & 3.32366000 & 3.87335900 & -0.38601600 & & \\
\hline $\mathrm{H}$ & 3.68512800 & 3.22650300 & -2.00041000 & & \\
\hline $\mathrm{H}$ & -1.16631500 & -4.11287300 & 0.49367200 & & \\
\hline $\mathrm{H}$ & -5.14263500 & 0.24453600 & -1.07697900 & & \\
\hline $\mathrm{H}$ & 1.29216400 & -2.33357100 & 1.35998300 & & \\
\hline $\mathrm{H}$ & 4.67991600 & -3.19400100 & -0.17052000 & & \\
\hline $\mathrm{H}$ & 4.99657700 & -1.89116600 & 1.00599700 & & \\
\hline $\mathrm{H}$ & 6.18655100 & -2.22588700 & -0.27880000 & & \\
\hline \multicolumn{6}{|c|}{ Anion } \\
\hline \multicolumn{4}{|c|}{ Cartesian Coordinates } & \multicolumn{2}{|l|}{ Frequency and Energy } \\
\hline 0 & -0.35726500 & -0.37822300 & -1.19579600 & \multirow{2}{*}{\multicolumn{2}{|c|}{$\begin{array}{l}\text { Zero-point correction= } \\
\text { Thermal correction to Energy= }\end{array}$}} \\
\hline $\mathrm{O}$ & 3.79717600 & -2.95252300 & 0.47533200 & Thermal correction to Energy= & \\
\hline 0 & 0.49398600 & 2.24539100 & -1.21777500 & \multicolumn{2}{|l|}{ Thermal correction to Enthalpy= } \\
\hline 0 & 0.75956500 & -3.07064500 & -1.06142100 & Thermal correction to Gibbs Free Energy= & 0.233191 \\
\hline 0 & 4.60819600 & 1.04234200 & 0.78052200 & Sum of electronic and zero-point Energies= & -1409.009646 \\
\hline $\mathrm{O}$ & 5.18703700 & -1.33492900 & 1.12601100 & Sum of electronic and thermal Energies= & -1408.984645 \\
\hline $\mathrm{O}$ & -4.43231500 & -1.33351700 & 1.15843600 & Sum of electronic and thermal Enthalpies= & -1408.983701 \\
\hline $\mathrm{O}$ & -1.27512500 & 3.53558700 & -1.08701800 & Sum of electronic and thermal Free Energies= & -1409.065011 \\
\hline $\mathrm{O}$ & -3.20640500 & -3.09218100 & -0.69173400 & & \\
\hline $\mathrm{C}$ & 1.78658500 & -1.06399500 & -0.23193200 & & \\
\hline $\mathrm{C}$ & 0.92710200 & -0.07429300 & -0.69986800 & & \\
\hline $\mathrm{C}$ & 3.06155000 & -0.69527500 & 0.24912000 & & \\
\hline $\mathrm{C}$ & 1.33534200 & -2.49393800 & -0.16344800 & & \\
\hline
\end{tabular}




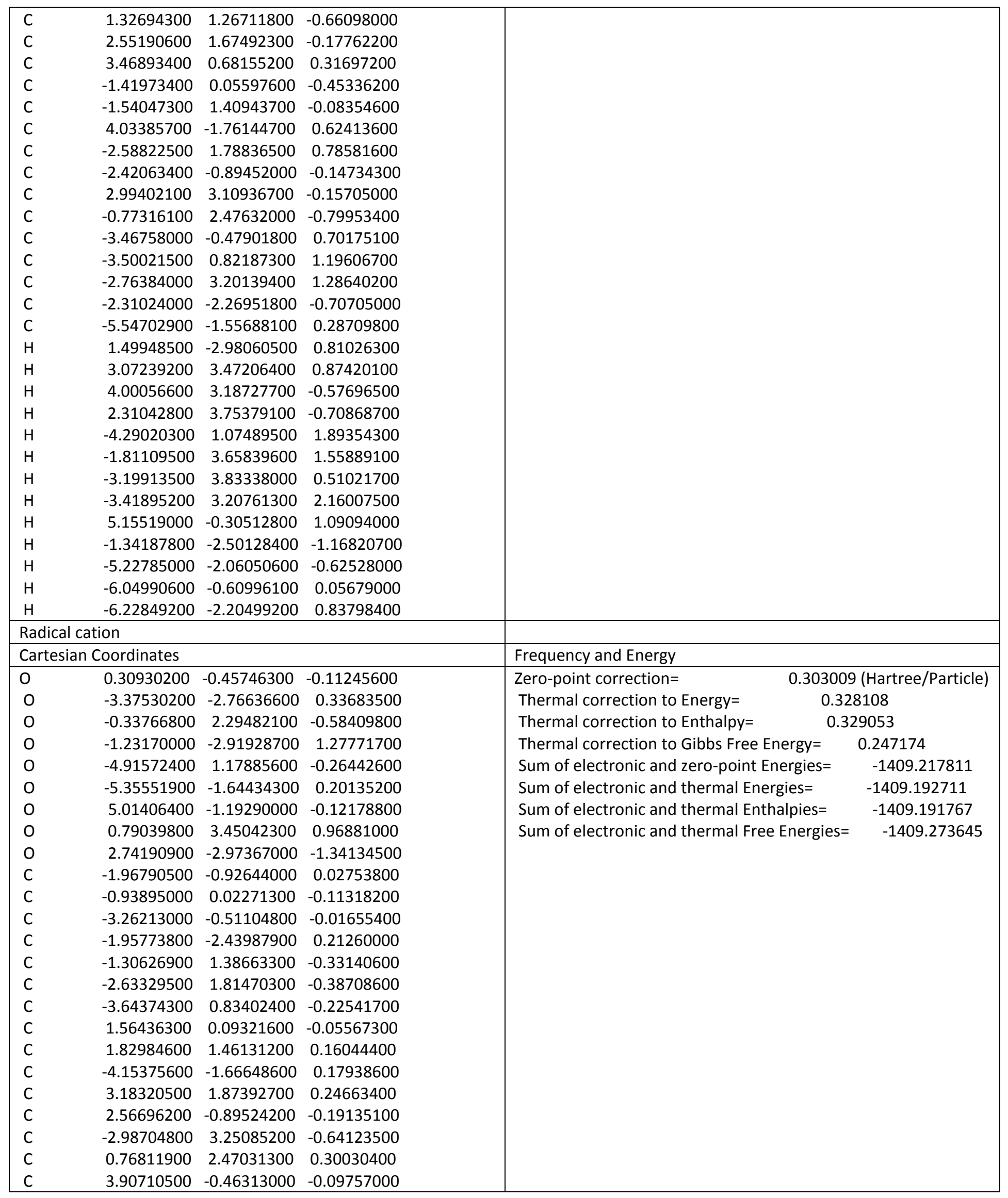




\begin{tabular}{|c|c|c|c|c|c|}
\hline \multirow{2}{*}{\multicolumn{3}{|c|}{$417210400 \quad 091364600 \quad 009289200$}} & & & \\
\hline & 4.17210400 & 0.91364600 & 0.09289200 & & \\
\hline C & 3.59034600 & 3.30337300 & 0.48547200 & & \\
\hline $\mathrm{C}$ & 2.14075700 & -2.28760200 & -0.55257300 & & \\
\hline C & 5.10061100 & -2.60686700 & 0.15963000 & & \\
\hline $\mathrm{H}$ & -1.58993400 & -2.95782300 & -0.67453200 & & \\
\hline $\mathrm{H}$ & -3.07148100 & 3.43921600 & -1.71662700 & & \\
\hline $\mathrm{H}$ & -3.94833800 & 3.49226800 & -0.18873800 & & \\
\hline $\mathrm{H}$ & -2.21946300 & 3.91508100 & -0.24761700 & & \\
\hline $\mathrm{H}$ & 5.21557600 & 1.19731900 & 0.14969700 & & \\
\hline $\mathrm{H}$ & 3.08864500 & 3.98910100 & -0.20044200 & & \\
\hline $\mathrm{H}$ & 3.32416500 & 3.62091800 & 1.49589600 & & \\
\hline $\mathrm{H}$ & 4.66641500 & 3.41158800 & 0.35673300 & & \\
\hline $\mathrm{H}$ & -1.58091000 & -2.56375300 & 2.10583600 & & \\
\hline $\mathrm{H}$ & -5.48237300 & 0.38907300 & -0.12469100 & & \\
\hline $\mathrm{H}$ & 1.21896100 & -2.64518500 & -0.06691600 & & \\
\hline $\mathrm{H}$ & 4.85362000 & -3.18562600 & -0.72704200 & & \\
\hline $\mathrm{H}$ & 4.43527300 & -2.87088400 & 0.98431800 & & \\
\hline $\mathrm{H}$ & 6.13626900 & -2.75996600 & 0.45220500 & & \\
\hline \multicolumn{6}{|c|}{ Name of compound (4) } \\
\hline \multicolumn{4}{|c|}{ Cartesian Coordinates } & \multicolumn{2}{|l|}{ Frequency and Energy } \\
\hline 0 & 0.22490800 & -0.20402200 & 1.29792000 & Zero-point correction= & (Hartree/Particle) \\
\hline $\mathrm{O}$ & -3.54623900 & -1.89507300 & -0.36352500 & Thermal correction to Energy= & 9107 \\
\hline 0 & 0.26040600 & 2.53396300 & 0.84010600 & Thermal correction to Enthalpy= & 20052 \\
\hline 0 & -2.17908400 & -2.41771800 & 1.46190300 & Thermal correction to Gibbs Free Energy= & 0.246199 \\
\hline $\mathrm{O}$ & -5.08213800 & -0.50872800 & -1.28089200 & Sum of electronic and zero-point Energies= & -1296.185035 \\
\hline $\mathrm{O}$ & 3.53177300 & -2.79718300 & -0.94689800 & Sum of electronic and thermal Energies= & -1296.162812 \\
\hline $\mathrm{O}$ & 2.36868100 & 3.13099700 & 0.98177600 & Sum of electronic and thermal Enthalpies= & -1296.161868 \\
\hline $\mathrm{C}$ & -1.92089000 & -0.32481400 & 0.18693600 & Sum of electronic and thermal Free Energies= & -1296.235720 \\
\hline $\mathrm{C}$ & -0.82215000 & 0.39631500 & 0.62529900 & & \\
\hline $\mathrm{C}$ & -2.97785200 & 0.33904600 & -0.41318900 & & \\
\hline $\mathrm{C}$ & -2.22252200 & -1.80672200 & 0.21293800 & & \\
\hline $\mathrm{C}$ & -0.78851500 & 1.77125200 & 0.39255000 & & \\
\hline $\mathrm{C}$ & -1.86049400 & 2.45460700 & -0.21672400 & & \\
\hline $\mathrm{C}$ & -2.97275400 & 1.71362500 & -0.61646200 & & \\
\hline $\mathrm{C}$ & 1.39851200 & -0.26390400 & 0.55299500 & & \\
\hline $\mathrm{C}$ & 2.04808800 & 0.92094900 & 0.17232800 & & \\
\hline $\mathrm{C}$ & -4.01664000 & -0.66026000 & -0.75957200 & & \\
\hline $\mathrm{C}$ & 3.24283000 & 0.81751800 & -0.58770900 & & \\
\hline $\mathrm{C}$ & 1.88855600 & -1.53016000 & 0.25769700 & & \\
\hline $\mathrm{C}$ & -1.78139500 & 3.94564900 & -0.40883200 & & \\
\hline $\mathrm{C}$ & 1.61751100 & 2.24598400 & 0.68636600 & & \\
\hline $\mathrm{C}$ & 3.03630400 & -1.60593600 & -0.54992200 & & \\
\hline $\mathrm{C}$ & 3.69116900 & -0.44359000 & -0.96029500 & & \\
\hline $\mathrm{C}$ & 4.03380300 & 2.02338800 & -1.03195200 & & \\
\hline $\mathrm{C}$ & 1.27299700 & -2.78427600 & 0.81229200 & & \\
\hline $\mathrm{H}$ & -1.55117900 & -2.38752100 & -0.42234800 & & \\
\hline $\mathrm{H}$ & -0.93467800 & 4.21667000 & -1.04510500 & & \\
\hline $\mathrm{H}$ & -2.69509000 & 4.32358500 & -0.86859300 & & \\
\hline $\mathrm{H}$ & -1.63093300 & 4.45634000 & 0.54552200 & & \\
\hline $\mathrm{H}$ & 4.57169700 & -0.55103200 & -1.58158800 & & \\
\hline $\mathrm{H}$ & 3.39501200 & 2.79696900 & -1.46271000 & & \\
\hline $\mathrm{H}$ & 4.54589900 & 2.48297300 & -0.18473500 & & \\
\hline $\mathrm{H}$ & 4.77498100 & 1.73000000 & -1.77645100 & & \\
\hline $\mathrm{H}$ & -2.68124600 & -1.88145600 & 2.08796200 & & \\
\hline
\end{tabular}




\begin{tabular}{|c|c|c|c|c|c|}
\hline 0 & 1.04514500 & -3.69725100 & -0.28976000 & & \\
\hline $\mathrm{H}$ & 0.70188100 & -4.52262700 & 0.06958100 & & \\
\hline $\mathrm{H}$ & 1.97327000 & -3.24534300 & 1.52199000 & & \\
\hline $\mathrm{H}$ & 0.34170200 & -2.56849800 & 1.33386800 & & \\
\hline $\mathrm{H}$ & -3.81954500 & 2.19784400 & -1.08846800 & & \\
\hline $\mathrm{H}$ & 2.82126000 & -3.45981100 & -0.86243100 & & \\
\hline $\operatorname{Rac}$ & & & & & \\
\hline Car & Coordinates & & & Frequency and Energy & \\
\hline 0 & -0.19975000 & -0.36595700 & -1.09958800 & Zero-point correction $=$ & (Hartree/Particle) \\
\hline $\mathrm{O}$ & 3.55370200 & -2.21942300 & 0.29255800 & Thermal correction to Energy= & 5691 \\
\hline 0 & 0.08461700 & 2.42075500 & -0.94906600 & Thermal correction to Enthalpy= & 06635 \\
\hline 0 & 1.58475200 & -2.98934300 & -0.57646400 & Thermal correction to Gibbs Free Energy= & 0.231883 \\
\hline 0 & 5.30402900 & -0.95076300 & 1.09224100 & Sum of electronic and zero-point Energies= & -1295.560119 \\
\hline 0 & -4.02591600 & -2.24049600 & 1.04741700 & Sum of electronic and thermal Energies= & -1295.537802 \\
\hline $\mathrm{O}$ & -1.92812800 & 3.27992400 & -1.05021700 & Sum of electronic and thermal Enthalpies= & -1295.536857 \\
\hline $\mathrm{C}$ & 2.03394000 & -0.59144000 & -0.17085900 & Sum of electronic and thermal Free Energies= & -1295.611610 \\
\hline $\mathrm{C}$ & 0.93720400 & 0.21045100 & -0.56709000 & & \\
\hline $\mathrm{C}$ & 3.20518700 & 0.05047000 & 0.33234900 & & \\
\hline $\mathrm{C}$ & 2.32312700 & -1.95459700 & -0.17497700 & & \\
\hline $\mathrm{C}$ & 1.05442500 & 1.57918200 & -0.45613900 & & \\
\hline $\mathrm{C}$ & 2.22060100 & 2.22400000 & 0.05462900 & & \\
\hline $\mathrm{C}$ & 3.29061000 & 1.43584000 & 0.45010500 & & \\
\hline $\mathrm{C}$ & -1.38465600 & -0.17698400 & -0.39297700 & & \\
\hline $\mathrm{C}$ & -1.84984300 & 1.11406900 & -0.08579800 & & \\
\hline C & 4.19329300 & -0.95528000 & 0.64884100 & & \\
\hline C & -3.04079700 & 1.23745200 & 0.67894700 & & \\
\hline $\mathrm{C}$ & -2.08988300 & -1.33270500 & -0.06007300 & & \\
\hline $\mathrm{C}$ & 2.26004100 & 3.72600400 & 0.12808300 & & \\
\hline $\mathrm{C}$ & -1.27613200 & 2.33167000 & -0.71146900 & & \\
\hline $\mathrm{C}$ & -3.28084200 & -1.17929600 & 0.67475600 & & \\
\hline $\mathrm{C}$ & -3.71275500 & 0.08851600 & 1.06694700 & & \\
\hline $\mathrm{C}$ & -3.61546800 & 2.57278700 & 1.08661700 & & \\
\hline $\mathrm{C}$ & -1.59857300 & -2.71744700 & -0.41539400 & & \\
\hline $\mathrm{H}$ & 1.46295300 & 4.11467700 & 0.76843300 & & \\
\hline $\mathrm{H}$ & 3.21767900 & 4.06470700 & 0.52532500 & & \\
\hline $\mathrm{H}$ & 2.11143600 & 4.17376900 & -0.85813100 & & \\
\hline $\mathrm{H}$ & -4.61869900 & 0.15354300 & 1.65695600 & & \\
\hline $\mathrm{H}$ & -2.84595300 & 3.25824000 & 1.44598700 & & \\
\hline $\mathrm{H}$ & -4.09536300 & 3.06358600 & 0.23827700 & & \\
\hline $\mathrm{H}$ & -4.35398200 & 2.43247800 & 1.87733200 & & \\
\hline $\mathrm{H}$ & 2.10504600 & -3.79633700 & -0.46041700 & & \\
\hline $\mathrm{O}$ & -2.67792000 & -3.58145200 & -0.83967800 & & \\
\hline $\mathrm{H}$ & -3.00394300 & -3.25571400 & -1.68662600 & & \\
\hline $\mathrm{H}$ & -0.81858800 & -2.67495700 & -1.17156100 & & \\
\hline $\mathrm{H}$ & -1.18183900 & -3.21816000 & 0.46199700 & & \\
\hline $\mathrm{H}$ & 4.19453300 & 1.88938500 & 0.83934600 & & \\
\hline $\mathrm{H}$ & -3.75323800 & -3.00733900 & 0.50646900 & & \\
\hline An & & & & & \\
\hline & Coordinates & & & Frequency and Energy & \\
\hline 0 & -0.27657600 & -0.12527100 & -1.44540100 & Zero-point correction= & (Hartree/Particle) \\
\hline $\mathrm{O}$ & 3.19507000 & -2.05286600 & 0.67019000 & Thermal correction to Energy= & 5807 \\
\hline 0 & -0.17371900 & 2.53771500 & -1.00269100 & Thermal correction to Enthalpy= & 66751 \\
\hline $\mathrm{O}$ & 1.68020100 & -2.75695900 & -0.95739300 & Thermal correction to Gibbs Free Energy= & 0.234822 \\
\hline 0 & 4.90976500 & -0.74965400 & 1.34994600 & Sum of electronic and zero-point Energies= & -1295.679007 \\
\hline
\end{tabular}




\begin{tabular}{|c|c|c|c|c|c|}
\hline 0 & -3.37577900 & -2.76901200 & 0.86630800 & Sum of electronic and thermal Energies= & -1295.657529 \\
\hline 0 & -2.14821500 & 3.36673600 & -0.44971200 & Sum of electronic and thermal Enthalpies= & -1295.656585 \\
\hline C & 1.80854200 & -0.40552500 & -0.22234600 & Sum of electronic and thermal Free Energies= & -1295.728514 \\
\hline C & 0.80732700 & 0.38133300 & -0.77200800 & & \\
\hline C & 2.92893500 & 0.20561400 & 0.31848300 & & \\
\hline C & 1.86046400 & -1.88443300 & 0.09211900 & & \\
\hline C & 0.87989100 & 1.76477300 & -0.61300200 & & \\
\hline C & 2.02356600 & 2.39482700 & -0.07941600 & & \\
\hline C & 3.07136300 & 1.58981600 & 0.36649300 & & \\
\hline C & -1.42222100 & -0.18314600 & -0.64086200 & & \\
\hline c & -1.94814300 & 1.01821600 & -0.08777600 & & \\
\hline C & 3.82309700 & -0.85052400 & 0.84154700 & & \\
\hline C & -3.08735600 & 0.86088400 & 0.78146100 & & \\
\hline C & -1.96331400 & -1.42676400 & -0.44868800 & & \\
\hline C & 2.06790000 & 3.89816000 & 0.00907000 & & \\
\hline C & -1.50920500 & 2.34330900 & -0.48878700 & & \\
\hline C & -3.01606800 & -1.60969500 & 0.52700900 & & \\
\hline C & -3.56198400 & -0.39596200 & 1.08070200 & & \\
\hline C & -3.75973200 & 2.05133400 & 1.42867000 & & \\
\hline C & -1.49148000 & -2.65416000 & -1.16920400 & & \\
\hline $\mathrm{H}$ & 1.14935800 & -2.10953300 & 0.89367500 & & \\
\hline $\mathrm{H}$ & 1.21787600 & 4.28325300 & 0.57787800 & & \\
\hline $\mathrm{H}$ & 2.99212800 & 4.23080200 & 0.48595600 & & \\
\hline $\mathrm{H}$ & 2.00466800 & 4.35297000 & -0.98392400 & & \\
\hline $\mathrm{H}$ & -4.37838900 & -0.51338400 & 1.78644800 & & \\
\hline $\mathrm{H}$ & -3.03869900 & 2.70798800 & 1.92255900 & & \\
\hline $\mathrm{H}$ & -4.27032600 & 2.66960900 & 0.68779500 & & \\
\hline $\mathrm{H}$ & -4.48533200 & 1.70752000 & 2.16936900 & & \\
\hline 0 & -0.87424000 & -3.61615900 & -0.28083400 & & \\
\hline $\mathrm{H}$ & 0.93261400 & -3.33191000 & -0.70725300 & & \\
\hline $\mathrm{H}$ & -2.35167600 & -3.13625000 & -1.64929800 & & \\
\hline $\mathrm{H}$ & -0.75033600 & -2.41133900 & -1.92641200 & & \\
\hline $\mathrm{H}$ & 3.96697800 & 2.02956400 & 0.79130900 & & \\
\hline $\mathrm{H}$ & -1.55042200 & -3.74386600 & 0.40780700 & & \\
\hline \multicolumn{6}{|c|}{ Radical cation } \\
\hline \multicolumn{4}{|c|}{ Cartesian Coordinates } & \multicolumn{2}{|l|}{ Frequency and Energy } \\
\hline $\mathrm{O}$ & 0.21621000 & -0.30798500 & 1.13959900 & \multicolumn{2}{|c|}{0.294629 (Hartree/Particle) } \\
\hline 0 & -3.43966900 & -2.22721800 & -0.47146400 & \multicolumn{2}{|l|}{ Thermal correction to Energy= } \\
\hline 0 & -0.05493000 & 2.49290800 & 0.91744700 & \multicolumn{2}{|l|}{ Thermal correction to Enthalpy= } \\
\hline 0 & -1.80463500 & -2.78564000 & 1.10760800 & \multicolumn{2}{|c|}{ Thermal correction to Gibbs Free Energy $=\quad 0.242441$} \\
\hline 0 & -5.17537300 & -0.94142300 & -1.16224200 & Sum of electronic and zero-point Energies= & -1295.888029 \\
\hline 0 & 4.07350700 & -2.18128700 & -0.86188500 & Sum of electronic and thermal Energies= & -1295.865407 \\
\hline 0 & 2.04062400 & 3.10287200 & 1.21949700 & Sum of electronic and thermal Enthalpies= & -1295.864463 \\
\hline C & -1.94842100 & -0.53850600 & 0.08976100 & Sum of electronic and thermal Free Energies= & -1295.940217 \\
\hline C & -0.91588800 & 0.26391700 & 0.53901500 & & \\
\hline C & -3.10187800 & 0.05686300 & -0.38842400 & & \\
\hline C & -2.08590300 & -2.04419800 & -0.03257700 & & \\
\hline C & -1.04241600 & 1.64651300 & 0.42902900 & & \\
\hline C & -2.20660800 & 2.26290500 & -0.06635600 & & \\
\hline C & -3.25620600 & 1.43459400 & -0.46930100 & & \\
\hline C & 1.38111600 & -0.18847900 & 0.44729900 & & \\
\hline C & 1.83585400 & 1.10418500 & 0.05379600 & & \\
\hline C & -4.06671900 & -1.02258700 & -0.73855500 & & \\
\hline C & 3.02120800 & 1.24789800 & -0.75625800 & & \\
\hline
\end{tabular}




\begin{tabular}{|c|c|c|c|c|c|}
\hline \multirow{2}{*}{\multicolumn{6}{|c|}{$\begin{array}{lll}2.12369500 & -1.32833200 & 0.23153300\end{array}$}} \\
\hline & & & & & \\
\hline C & -2.30460800 & 3.76381300 & -0.12180900 & & \\
\hline C & 1.26922400 & 2.31408300 & 0.74910700 & & \\
\hline C & 3.32509400 & -1.16554700 & -0.54606300 & & \\
\hline C & 3.72434900 & 0.11002800 & -1.04994500 & & \\
\hline C & 3.47778600 & 2.58472500 & -1.26707400 & & \\
\hline C & 1.72120800 & -2.64686900 & 0.84250100 & & \\
\hline $\mathrm{H}$ & -1.43371800 & -2.44911900 & -0.81088300 & & \\
\hline $\mathrm{H}$ & -1.53233400 & 4.18883600 & -0.76834800 & & \\
\hline $\mathrm{H}$ & -3.27742700 & 4.06884000 & -0.50549200 & & \\
\hline $\mathrm{H}$ & -2.16868500 & 4.20313100 & 0.86944100 & & \\
\hline $\mathrm{H}$ & 4.61411100 & 0.13554800 & -1.66653300 & & \\
\hline $\mathrm{H}$ & 2.64786800 & 3.15644500 & -1.68836800 & & \\
\hline $\mathrm{H}$ & 3.89619600 & 3.17967700 & -0.45277800 & & \\
\hline $\mathrm{H}$ & 4.23742900 & 2.45642800 & -2.03717100 & & \\
\hline $\mathrm{H}$ & -2.39260100 & -2.50954600 & 1.82316400 & & \\
\hline $\mathrm{O}$ & 2.31313000 & -3.70449400 & 0.07277900 & & \\
\hline $\mathrm{H}$ & 2.17886900 & -4.55511400 & 0.50604600 & & \\
\hline $\mathrm{H}$ & 2.08827300 & -2.67605200 & 1.87794400 & & \\
\hline $\mathrm{H}$ & 0.63323100 & -2.74347900 & 0.87686700 & & \\
\hline $\mathrm{H}$ & -4.18046200 & 1.85859700 & -0.84336000 & & \\
\hline $\mathrm{H}$ & 3.63300200 & -3.02609000 & -0.54531200 & & \\
\hline \multicolumn{6}{|c|}{ Name of compound (5) } \\
\hline \multicolumn{4}{|c|}{ Cartesian Coordinates } & \multicolumn{2}{|l|}{ Frequency and Energy } \\
\hline $\mathrm{O}$ & 0.24440100 & -0.09655100 & 1.46610700 & Zero-point correction $=$ & (Hartree/Particle) \\
\hline $\mathrm{O}$ & -3.08623000 & -2.63684400 & -0.18280200 & Thermal correction to Energy= & 4527 \\
\hline $\mathrm{O}$ & -0.35262600 & 2.54974500 & 0.91295900 & Thermal correction to Enthalpy= & 5472 \\
\hline $\mathrm{O}$ & -1.76607800 & -2.74985200 & 1.75569600 & Thermal correction to Gibbs Free Energy= & 0.275186 \\
\hline $\mathrm{O}$ & -4.36407700 & 1.30751800 & -1.30319100 & Sum of electronic and zero-point Energies= & -1410.709616 \\
\hline $\mathrm{O}$ & -4.79628900 & -1.58224300 & -1.24486500 & Sum of electronic and thermal Energies= & -1410.684544 \\
\hline 0 & 3.98952900 & -1.98713100 & -0.65890700 & Sum of electronic and thermal Enthalpies= & -1410.683600 \\
\hline 0 & 1.55493000 & 3.60896200 & 1.14938800 & Sum of electronic and thermal Free Energies= & -1410.763886 \\
\hline $\mathrm{C}$ & -1.79643800 & -0.74926600 & 0.33302900 & & \\
\hline $\mathrm{C}$ & -0.90288500 & 0.21271000 & 0.75365300 & & \\
\hline $\mathrm{C}$ & -2.94504100 & -0.35944600 & -0.33677800 & & \\
\hline $\mathrm{C}$ & -1.81944400 & -2.26038400 & 0.46259800 & & \\
\hline $\mathrm{C}$ & -1.18317900 & 1.55546500 & 0.44764200 & & \\
\hline $\mathrm{C}$ & -2.33839700 & 1.96614600 & -0.23474600 & & \\
\hline $\mathrm{C}$ & -3.24155000 & 0.96682200 & -0.63846000 & & \\
\hline $\mathrm{C}$ & 1.40614700 & 0.08575200 & 0.72786500 & & \\
\hline $\mathrm{C}$ & 1.77559600 & 1.38641700 & 0.34076400 & & \\
\hline $\mathrm{C}$ & -3.73826100 & -1.54362700 & -0.65870600 & & \\
\hline $\mathrm{C}$ & 2.96217400 & 1.55820700 & -0.40294000 & & \\
\hline $\mathrm{C}$ & 2.16455400 & -1.03955100 & 0.43848600 & & \\
\hline $\mathrm{C}$ & -2.62459800 & 3.41236400 & -0.53393700 & & \\
\hline $\mathrm{C}$ & 1.03128000 & 2.57978500 & 0.82778600 & & \\
\hline $\mathrm{C}$ & 3.31917800 & -0.84558500 & -0.33870600 & & \\
\hline $\mathrm{C}$ & 3.70439100 & 0.42874600 & -0.75299300 & & \\
\hline $\mathrm{C}$ & 3.46149300 & 2.91187200 & -0.84572900 & & \\
\hline $\mathrm{C}$ & 1.77044500 & -2.43101800 & 0.87919800 & & \\
\hline $\mathrm{C}$ & 5.20815600 & -1.89600300 & -1.39601900 & & \\
\hline $\mathrm{H}$ & -1.02196700 & -2.75696700 & -0.08979700 & & \\
\hline $\mathrm{H}$ & -2.45794000 & 3.62816300 & -1.59445700 & & \\
\hline $\mathrm{H}$ & -3.67143600 & 3.64166100 & -0.32525600 & & \\
\hline
\end{tabular}




\begin{tabular}{|c|c|c|c|c|c|}
\hline $\mathrm{H}$ & -1.98661700 & 4.06794300 & 0.05507000 & & \\
\hline $\mathrm{H}$ & 4.59502200 & 0.56325200 & -1.35112200 & & \\
\hline $\mathrm{H}$ & 2.66748300 & 3.51508200 & -1.29006100 & & \\
\hline $\mathrm{H}$ & 3.84069900 & 3.48050500 & 0.00521800 & & \\
\hline $\mathrm{H}$ & 4.26135200 & 2.79807000 & -1.57913500 & & \\
\hline $\mathrm{H}$ & -2.37136200 & -2.24053500 & 2.30857900 & & \\
\hline $\mathrm{H}$ & -4.86066600 & 0.48980900 & -1.49265500 & & \\
\hline $\mathrm{H}$ & 5.95187500 & -1.30333700 & -0.85551000 & & \\
\hline $\mathrm{H}$ & 5.04026500 & -1.46525900 & -2.38782400 & & \\
\hline $\mathrm{H}$ & 5.56543200 & -2.91848900 & -1.50047600 & & \\
\hline $\mathrm{O}$ & 1.27199000 & -3.22422500 & -0.20315300 & & \\
\hline $\mathrm{H}$ & 2.00171900 & -3.34938500 & -0.81929500 & & \\
\hline $\mathrm{H}$ & 2.63217800 & -2.92127300 & 1.34432100 & & \\
\hline $\mathrm{H}$ & 0.96782300 & -2.38071300 & 1.61051500 & & \\
\hline \multicolumn{6}{|c|}{ Radical } \\
\hline \multicolumn{4}{|c|}{ Cartesian Coordinates } & \multicolumn{2}{|l|}{ Frequency and Energy } \\
\hline $\mathrm{O}$ & 0.21437500 & -0.19874600 & 1.34067300 & Zero-point correction $=$ & (Hartree/Particle) \\
\hline $\mathrm{O}$ & -3.11061000 & -2.74766100 & -0.28027000 & Thermal correction to Energy= & 1142 \\
\hline $\mathrm{O}$ & -0.48918800 & 2.46313800 & 0.94609500 & Thermal correction to Enthalpy= & 42086 \\
\hline $\mathrm{O}$ & -1.64955600 & -2.92013500 & 1.54935000 & Thermal correction to Gibbs Free Energy= & 0.260986 \\
\hline $\mathrm{O}$ & -4.53465500 & 1.20596400 & -1.18421000 & Sum of electronic and zero-point Energies= & -1410.084389 \\
\hline 0 & -4.89629500 & -1.70000700 & -1.21775800 & Sum of electronic and thermal Energies= & -1410.059477 \\
\hline $\mathrm{O}$ & 4.18663200 & -1.76560300 & -0.73962700 & Sum of electronic and thermal Enthalpies= & -1410.058532 \\
\hline 0 & 1.37801500 & 3.57041500 & 1.25300200 & Sum of electronic and thermal Free Energies= & -1410.139632 \\
\hline $\mathrm{C}$ & -1.84052600 & -0.84727800 & 0.23951100 & & \\
\hline $\mathrm{C}$ & -0.95363800 & 0.11675700 & 0.66983900 & & \\
\hline $\mathrm{C}$ & -3.02780500 & -0.46267600 & -0.36410800 & & \\
\hline $\mathrm{C}$ & -1.81909700 & -2.36185200 & 0.29628200 & & \\
\hline $\mathrm{C}$ & -1.29032900 & 1.46405100 & 0.44886600 & & \\
\hline $\mathrm{C}$ & -2.48301500 & 1.87074600 & -0.16952100 & & \\
\hline $\mathrm{C}$ & -3.37443800 & 0.86689400 & -0.58703200 & & \\
\hline C & 1.37704900 & 0.09399800 & 0.63904300 & & \\
\hline C & 1.68770700 & 1.41155400 & 0.31570600 & & \\
\hline $\mathrm{C}$ & -3.81108200 & -1.65178200 & -0.68701500 & & \\
\hline $\mathrm{C}$ & 2.87477200 & 1.69064700 & -0.40965200 & & \\
\hline $\mathrm{C}$ & 2.21193400 & -1.01759800 & 0.32888100 & & \\
\hline $\mathrm{C}$ & -2.82153200 & 3.32172400 & -0.37734700 & & \\
\hline $\mathrm{C}$ & 0.89394700 & 2.54532600 & 0.86693800 & & \\
\hline $\mathrm{C}$ & 3.39367900 & -0.69206500 & -0.41461400 & & \\
\hline $\mathrm{C}$ & 3.68754900 & 0.60931000 & -0.77798800 & & \\
\hline $\mathrm{C}$ & 3.29752100 & 3.08461900 & -0.79870800 & & \\
\hline $\mathrm{C}$ & 1.85805400 & -2.30872400 & 0.75704200 & & \\
\hline $\mathrm{C}$ & 5.39413000 & -1.55373200 & -1.47092400 & & \\
\hline $\mathrm{H}$ & -1.05518100 & -2.80664800 & -0.34134200 & & \\
\hline $\mathrm{H}$ & -3.88356600 & 3.49177100 & -0.19343100 & & \\
\hline $\mathrm{H}$ & -2.23206400 & 3.95890900 & 0.27885900 & & \\
\hline $\mathrm{H}$ & -2.62437300 & 3.62053100 & -1.41240900 & & \\
\hline $\mathrm{H}$ & 4.58236200 & 0.81840200 & -1.34806500 & & \\
\hline $\mathrm{H}$ & 2.47212200 & 3.66342400 & -1.21951100 & & \\
\hline $\mathrm{H}$ & 3.65699200 & 3.64555500 & 0.06707000 & & \\
\hline $\mathrm{H}$ & 4.09628700 & 3.04050800 & -1.54164900 & & \\
\hline $\mathrm{H}$ & -2.20651600 & -2.44990300 & 2.18193600 & & \\
\hline $\mathrm{H}$ & -5.02138400 & 0.38581800 & -1.38609600 & & \\
\hline $\mathrm{H}$ & 6.07899700 & -0.90672500 & -0.91566000 & & \\
\hline
\end{tabular}




\begin{tabular}{|c|c|c|c|c|c|}
\hline $\mathrm{H}$ & 5.18573700 & -1.12083700 & -2.45341700 & & \\
\hline $\mathrm{H}$ & 5.84190300 & -2.53783300 & -1.59471400 & & \\
\hline 0 & 2.55926600 & -3.43325500 & 0.52008200 & & \\
\hline $\mathrm{H}$ & 3.34665000 & -3.19802000 & 0.00290900 & & \\
\hline $\mathrm{H}$ & 0.96888300 & -2.47734800 & 1.34562200 & & \\
\hline \multicolumn{6}{|c|}{ Anion } \\
\hline \multicolumn{4}{|c|}{ Cartesian Coordinates } & \multicolumn{2}{|l|}{ Frequency and Energy } \\
\hline $\mathrm{O}$ & -0.27318200 & -0.20509500 & -1.44013800 & Zero-point correction $=$ & 0.313018 (Hartree/Particle) \\
\hline $\mathrm{O}$ & 3.83341900 & -2.79533700 & 0.30924200 & Thermal correction to Energy= & 0.338345 \\
\hline 0 & 0.44029800 & 2.43828100 & -1.07099000 & Thermal correction to Enthalpy= & 0.339289 \\
\hline 0 & 1.14113800 & -2.78298300 & -1.72549400 & Thermal correction to Gibbs Free Energy= & 0.257655 \\
\hline $\mathrm{O}$ & 4.37517300 & 1.15453600 & 1.22221600 & Sum of electronic and zero-point Energies= & -1410.196909 \\
\hline $\mathrm{O}$ & 5.05661000 & -1.22028400 & 1.30919900 & Sum of electronic and thermal Energies= & -1410.171582 \\
\hline 0 & -4.18961000 & -1.76476100 & 0.66834200 & Sum of electronic and thermal Enthalpies= & -1410.170638 \\
\hline 0 & -1.42773900 & 3.57969300 & -1.18315200 & \multirow{36}{*}{\multicolumn{2}{|c|}{ Sum of electronic and thermal Free Energies= }} \\
\hline $\mathrm{C}$ & 1.82047600 & -0.91419200 & -0.39298500 & & \\
\hline $\mathrm{C}$ & 0.94881600 & 0.08728300 & -0.80291400 & & \\
\hline $\mathrm{C}$ & 3.00895500 & -0.55950800 & 0.27817700 & & \\
\hline $\mathrm{C}$ & 1.47086600 & -2.35737000 & -0.64023700 & & \\
\hline $\mathrm{C}$ & 1.25782300 & 1.42956100 & -0.54389800 & & \\
\hline $\mathrm{C}$ & 2.40023400 & 1.82144400 & 0.12248500 & & \\
\hline $\mathrm{C}$ & 3.31627900 & 0.81139300 & 0.58323200 & & \\
\hline $\mathrm{C}$ & -1.38953500 & 0.07121800 & -0.68921100 & & \\
\hline $\mathrm{C}$ & -1.66046500 & 1.39766700 & -0.28707700 & & \\
\hline C & 3.98944100 & -1.62432500 & 0.62746900 & & \\
\hline c & -2.79451900 & 1.65778000 & 0.50494000 & & \\
\hline $\mathrm{C}$ & -2.26310200 & -0.98136000 & -0.40765400 & & \\
\hline $\mathrm{C}$ & 2.75171700 & 3.25843000 & 0.38058900 & & \\
\hline $\mathrm{C}$ & -0.89395600 & 2.54443300 & -0.86219300 & & \\
\hline $\mathrm{C}$ & -3.38110500 & -0.68898800 & 0.38613500 & & \\
\hline $\mathrm{C}$ & -3.62778300 & 0.59669400 & 0.86066800 & & \\
\hline $\mathrm{C}$ & -3.15360600 & 3.04208900 & 0.99309700 & & \\
\hline $\mathrm{C}$ & -2.05358300 & -2.39272700 & -0.91772200 & & \\
\hline $\mathrm{C}$ & -5.36126700 & -1.55938900 & 1.44328800 & & \\
\hline $\mathrm{H}$ & 1.45779100 & -2.99765700 & 0.25488400 & & \\
\hline $\mathrm{H}$ & 2.68863200 & 3.48443900 & 1.45143700 & & \\
\hline $\mathrm{H}$ & 3.79322700 & 3.43698300 & 0.09956000 & & \\
\hline $\mathrm{H}$ & 2.09838300 & 3.93981800 & -0.16367200 & & \\
\hline $\mathrm{H}$ & -4.48134800 & 0.79797100 & 1.49370800 & & \\
\hline $\mathrm{H}$ & -2.28283400 & 3.57631300 & 1.37743600 & & \\
\hline $\mathrm{H}$ & -3.55229400 & 3.64911800 & 0.17848000 & & \\
\hline $\mathrm{H}$ & -3.90019000 & 2.97773300 & 1.78838100 & & \\
\hline $\mathrm{H}$ & 4.96635700 & -0.19721500 & 1.40538500 & & \\
\hline $\mathrm{H}$ & -6.04362800 & -0.85423400 & 0.95670800 & & \\
\hline $\mathrm{H}$ & -5.12063100 & -1.19688700 & 2.44858700 & & \\
\hline $\mathrm{H}$ & -5.84307800 & -2.53355900 & 1.51770400 & & \\
\hline 0 & -1.87365500 & -3.34332100 & 0.13295900 & & \\
\hline $\mathrm{H}$ & -2.67256600 & -3.29949800 & 0.66843700 & & \\
\hline $\mathrm{H}$ & -2.91942500 & -2.66952000 & -1.53657300 & & \\
\hline $\mathrm{H}$ & -1.16212300 & -2.44043500 & -1.53506300 & & \\
\hline \multicolumn{6}{|c|}{ Radical cation } \\
\hline \multicolumn{4}{|c|}{ Cartesian Coordinates } & \multicolumn{2}{|l|}{ Frequency and Energy } \\
\hline $\mathrm{O}$ & 0.23465500 & -0.41967300 & 0.61496200 & Zero-point correction $=$ & \multirow{2}{*}{0.327558 (Hartree/Particle) } \\
\hline $\mathrm{O}$ & -3.55727300 & -2.51351800 & 0.20702500 & Thermal correction to Energy= & \\
\hline
\end{tabular}




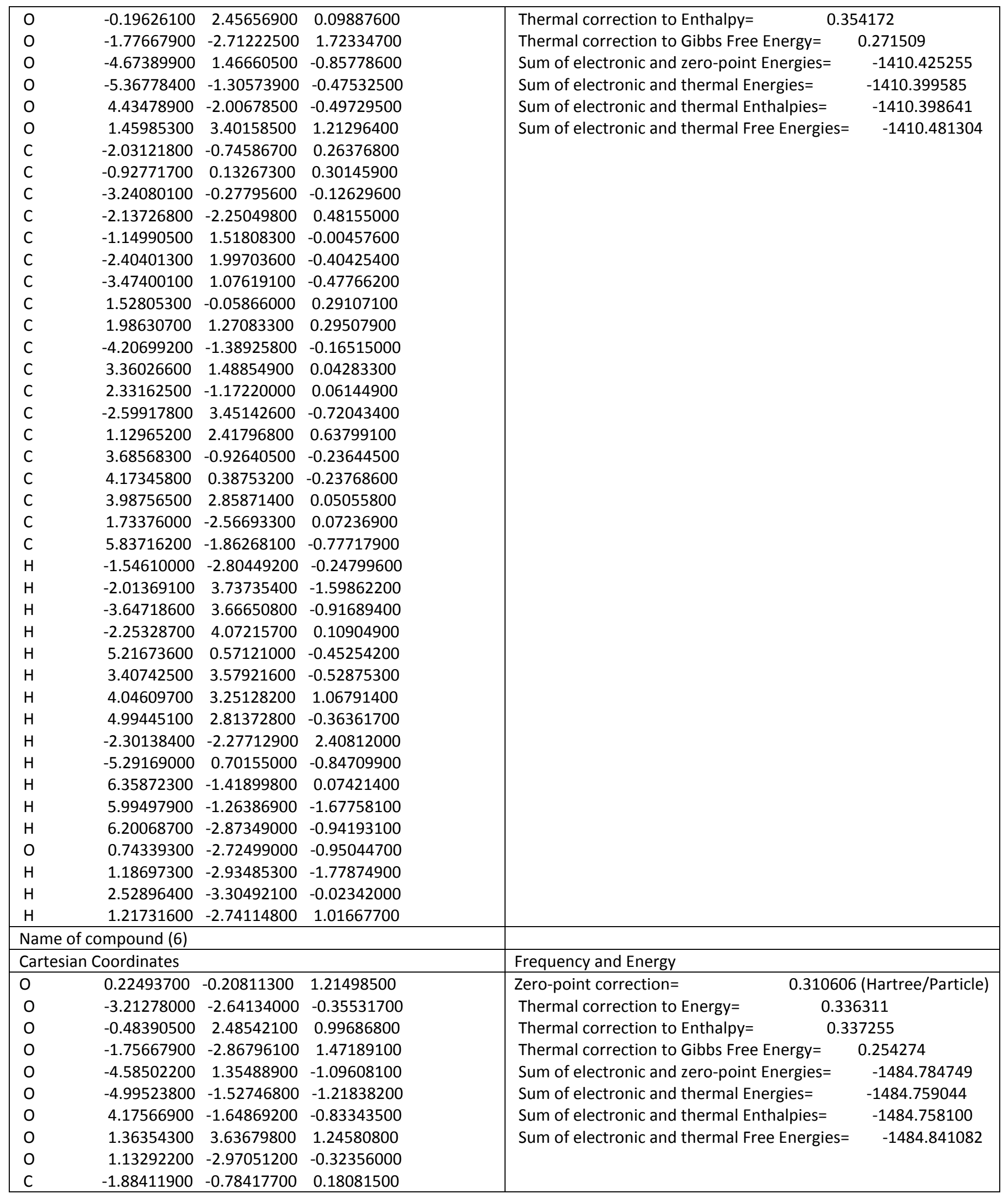




\begin{tabular}{|c|c|c|c|c|c|}
\hline \multirow{2}{*}{\multicolumn{6}{|c|}{$\begin{array}{lll}-0.96858000 & 0.15501300 & 0.60929000\end{array}$}} \\
\hline & & & & & \\
\hline C & -3.08254600 & -0.35900500 & -0.36939400 & & \\
\hline C & -1.90141200 & -2.30157800 & 0.21929100 & & \\
\hline C & -1.29833800 & 1.51295900 & 0.46179400 & & \\
\hline C & -2.50170800 & 1.95825500 & -0.10733600 & & \\
\hline C & -3.41470300 & 0.98167500 & -0.54107100 & & \\
\hline C & 1.37108600 & 0.14839200 & 0.52666800 & & \\
\hline C & 1.68296100 & 1.49574200 & 0.27411400 & & \\
\hline C & -3.89655900 & -1.52356700 & -0.71080300 & & \\
\hline C & 2.88637600 & 1.78959400 & -0.40904500 & & \\
\hline C & 2.21330400 & -0.88775300 & 0.15853600 & & \\
\hline C & -2.83099000 & 3.41971500 & -0.24554700 & & \\
\hline C & 0.88772800 & 2.60509700 & 0.86237600 & & \\
\hline C & 3.40839800 & -0.58614800 & -0.50811300 & & \\
\hline C & 3.71684500 & 0.74238200 & -0.80651700 & & \\
\hline C & 3.31992700 & 3.19927800 & -0.73061300 & & \\
\hline C & 1.82517700 & -2.31653200 & 0.41347000 & & \\
\hline C & 5.40007300 & -1.43802400 & -1.53511700 & & \\
\hline $\mathrm{H}$ & -1.14903400 & -2.76133000 & -0.41706900 & & \\
\hline $\mathrm{H}$ & -2.66624600 & 3.75717100 & -1.27435600 & & \\
\hline $\mathrm{H}$ & -3.88449800 & 3.59199600 & -0.01921100 & & \\
\hline $\mathrm{H}$ & -2.21351000 & 4.02503200 & 0.41501900 & & \\
\hline $\mathrm{H}$ & 4.62625400 & 0.98056600 & -1.34013500 & & \\
\hline $\mathrm{H}$ & 2.50057000 & 3.79716100 & -1.13298200 & & \\
\hline $\mathrm{H}$ & 3.66247900 & 3.71256100 & 0.16953000 & & \\
\hline $\mathrm{H}$ & 4.13136500 & 3.18481200 & -1.45993800 & & \\
\hline $\mathrm{H}$ & -2.32188400 & -2.39599100 & 2.09555600 & & \\
\hline $\mathrm{H}$ & -5.08552700 & 0.54620700 & -1.31336000 & & \\
\hline $\mathrm{H}$ & 6.09071900 & -0.82435700 & -0.94908000 & & \\
\hline $\mathrm{H}$ & 5.22219900 & -0.97287900 & -2.50929500 & & \\
\hline $\mathrm{H}$ & 5.82636900 & -2.42840000 & -1.67862500 & & \\
\hline 0 & 2.34131200 & -2.78579400 & 1.55980700 & & \\
\hline $\mathrm{H}$ & 2.02514600 & -3.69827600 & 1.65648200 & & \\
\hline \multicolumn{6}{|c|}{ Radical } \\
\hline \multicolumn{4}{|c|}{ Cartesian Coordinates } & \multicolumn{2}{|l|}{ Frequency and Energy } \\
\hline O & 0.20928700 & -0.32210100 & 1.12925600 & Zero-point correction= & (Hartree/Particle) \\
\hline $\mathrm{O}$ & -3.08840400 & -2.81565300 & -0.57225700 & Thermal correction to Energy= & 2871 \\
\hline 0 & -0.68950900 & 2.30928000 & 1.16322600 & Thermal correction to Enthalpy= & 3816 \\
\hline $\mathrm{O}$ & -1.02584100 & -3.32514100 & 0.11393700 & Thermal correction to Gibbs Free Energy= & 0.242440 \\
\hline $\mathrm{O}$ & -4.88693500 & 1.08836600 & -0.69005900 & Sum of electronic and zero-point Energies= & -1484.167231 \\
\hline 0 & -5.07871300 & -1.82573300 & -1.17276300 & Sum of electronic and thermal Energies= & -1484.142151 \\
\hline $\mathrm{O}$ & 4.41099800 & -1.24125800 & -0.75566000 & Sum of electronic and thermal Enthalpies= & -1484.141207 \\
\hline $\mathrm{O}$ & 1.02122000 & 3.67069200 & 0.98914100 & Sum of electronic and thermal Free Energies= & -1484.222583 \\
\hline $\mathrm{O}$ & 1.41296400 & -2.87063800 & 0.89410800 & & \\
\hline C & -1.92036000 & -0.98578700 & 0.12485300 & & \\
\hline $\mathrm{C}$ & -1.04492200 & 0.00807500 & 0.61533500 & & \\
\hline $\mathrm{C}$ & -3.21507400 & -0.56798400 & -0.29595900 & & \\
\hline $\mathrm{C}$ & -1.90516000 & -2.37730800 & -0.07191100 & & \\
\hline $\mathrm{C}$ & -1.48733400 & 1.31597300 & 0.62423300 & & \\
\hline C & -2.77062800 & 1.74309200 & 0.19430400 & & \\
\hline C & -3.64709400 & 0.75862700 & -0.27136200 & & \\
\hline C & 1.30639900 & 0.16340500 & 0.46563200 & & \\
\hline C & 1.40908100 & 1.51754300 & 0.08852600 & & \\
\hline $\mathrm{C}$ & -3.95296600 & -1.71076100 & -0.73546200 & & \\
\hline
\end{tabular}




\begin{tabular}{|c|c|c|c|c|c|}
\hline \multirow{2}{*}{\multicolumn{6}{|c|}{$\begin{array}{lll}2.51508500 & 1.91280300 & -0.70261600\end{array}$}} \\
\hline & & & & & \\
\hline C & 2.36196100 & -0.73576400 & 0.26294900 & & \\
\hline C & -3.16300200 & 3.19310900 & 0.26241400 & & \\
\hline C & 0.58671700 & 2.57941900 & 0.74123900 & & \\
\hline C & 3.46433700 & -0.31623700 & -0.51110700 & & \\
\hline C & 3.49270600 & 0.97873000 & -1.03010200 & & \\
\hline C & 2.68709100 & 3.31947600 & -1.22081700 & & \\
\hline C & 2.33840400 & -2.08188700 & 0.89546400 & & \\
\hline $\mathrm{C}$ & 5.56489800 & -0.88458200 & -1.51731900 & & \\
\hline $\mathrm{H}$ & -4.20577700 & 3.31323100 & -0.02638800 & & \\
\hline $\mathrm{H}$ & -3.02591800 & 3.59058100 & 1.27098500 & & \\
\hline $\mathrm{H}$ & -2.54815200 & 3.80507000 & -0.40490500 & & \\
\hline $\mathrm{H}$ & 4.30798200 & 1.28714100 & -1.66853400 & & \\
\hline $\mathrm{H}$ & 1.74352300 & 3.75140500 & -1.55589600 & & \\
\hline $\mathrm{H}$ & 3.06574200 & 3.97367700 & -0.43355100 & & \\
\hline $\mathrm{H}$ & 3.39133300 & 3.32564300 & -2.05452400 & & \\
\hline $\mathrm{H}$ & -0.14474200 & -2.99017900 & 0.41424100 & & \\
\hline $\mathrm{H}$ & -5.34001300 & 0.27310500 & -0.97075000 & & \\
\hline $\mathrm{H}$ & 6.11404000 & -0.06719700 & -1.04161100 & & \\
\hline $\mathrm{H}$ & 5.29456200 & -0.60797700 & -2.54066100 & & \\
\hline $\mathrm{H}$ & 6.18493700 & -1.77802700 & -1.53528000 & & \\
\hline 0 & 3.47373800 & -2.36716500 & 1.54264900 & & \\
\hline $\mathrm{H}$ & 3.37120200 & -3.26134000 & 1.90449900 & & \\
\hline \multicolumn{6}{|c|}{ 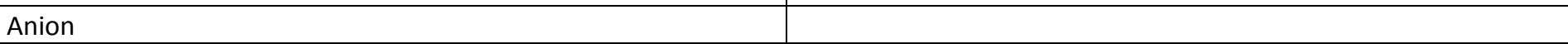 } \\
\hline \multicolumn{4}{|c|}{ Cartesian Coordinates } & \multicolumn{2}{|l|}{ Frequency and Energy } \\
\hline $\mathrm{O}$ & 0.25309900 & -0.46093400 & 1.00374000 & Zero-point correction $=$ & (Hartree/Particle) \\
\hline $\mathrm{O}$ & -3.97357700 & -2.94107500 & -0.78912000 & Thermal correction to Energy= & 974 \\
\hline $\mathrm{O}$ & -0.58098700 & 2.10630800 & 1.29241200 & Thermal correction to Enthalpy= & 0918 \\
\hline $\mathrm{O}$ & -0.26046200 & -2.84678000 & -0.37207300 & Thermal correction to Gibbs Free Energy $=$ & 0.239377 \\
\hline $\mathrm{O}$ & -4.89469500 & 1.00388200 & -0.31116600 & Sum of electronic and zero-point Energies= & -1484.280850 \\
\hline $\mathrm{O}$ & -5.43375500 & -1.27987200 & -1.02096200 & Sum of electronic and thermal Energies= & -1484.255533 \\
\hline $\mathrm{O}$ & 4.57851100 & -1.15962800 & -0.60654000 & Sum of electronic and thermal Enthalpies= & -1484.254589 \\
\hline $\mathrm{O}$ & 0.80446700 & 3.65761500 & 0.55828200 & Sum of electronic and thermal Free Energies= & -1484.336130 \\
\hline $\mathrm{O}$ & 3.66853900 & -1.99447800 & 1.92848800 & & \\
\hline $\mathrm{C}$ & -1.87666600 & -1.07061900 & -0.03537300 & & \\
\hline $\mathrm{C}$ & -1.02878000 & -0.12764400 & 0.55790400 & & \\
\hline $\mathrm{C}$ & -3.22919200 & -0.71150800 & -0.29475800 & & \\
\hline $\mathrm{C}$ & -1.33313300 & -2.31317600 & -0.62747200 & & \\
\hline $\mathrm{C}$ & -1.47338000 & 1.17943300 & 0.75606500 & & \\
\hline C & -2.75682900 & 1.58878000 & 0.46179100 & & \\
\hline $\mathrm{C}$ & -3.69507100 & 0.62858100 & -0.05928600 & & \\
\hline $\mathrm{C}$ & 1.34579000 & 0.09355400 & 0.40348600 & & \\
\hline $\mathrm{C}$ & 1.36630100 & 1.43491200 & -0.06386700 & & \\
\hline $\mathrm{C}$ & -4.22703400 & -1.74632700 & -0.72654100 & & \\
\hline $\mathrm{C}$ & 2.44703600 & 1.82838200 & -0.89003600 & & \\
\hline $\mathrm{C}$ & 2.47709400 & -0.72823000 & 0.33540700 & & \\
\hline $\mathrm{C}$ & -3.23984000 & 2.99094100 & 0.68133700 & & \\
\hline $\mathrm{C}$ & 0.51618700 & 2.48684700 & 0.55384200 & & \\
\hline $\mathrm{C}$ & 3.54524900 & -0.29748500 & -0.46961400 & & \\
\hline C & 3.48330900 & 0.93321200 & -1.13092400 & & \\
\hline C & 2.53565100 & 3.19124300 & -1.53598300 & & \\
\hline C & 2.66991200 & -1.92819800 & 1.25159200 & & \\
\hline $\mathrm{C}$ & 5.71750000 & -0.76806800 & -1.35483700 & & \\
\hline $\mathrm{H}$ & -1.95311700 & -2.73726200 & -1.42165500 & & \\
\hline
\end{tabular}




\begin{tabular}{|c|c|c|c|c|c|}
\hline $\mathrm{H}$ & -4.12351300 & 2.97961100 & 1.32683700 & & \\
\hline $\mathrm{H}$ & -2.46800800 & 3.62101700 & 1.12050500 & & \\
\hline $\mathrm{H}$ & -3.57225400 & 3.42670300 & -0.26653300 & & \\
\hline $\mathrm{H}$ & 4.27784100 & 1.23097700 & -1.80078900 & & \\
\hline $\mathrm{H}$ & 1.57115300 & 3.52619000 & -1.91922100 & & \\
\hline $\mathrm{H}$ & 2.85750600 & 3.94416700 & -0.81423400 & & \\
\hline $\mathrm{H}$ & 3.25241000 & 3.16086300 & -2.36040900 & & \\
\hline $\mathrm{H}$ & -5.42239800 & -0.26849500 & -0.80517500 & & \\
\hline $\mathrm{H}$ & 6.17144100 & 0.14216300 & -0.94845500 & & \\
\hline $\mathrm{H}$ & 5.47517600 & -0.61378800 & -2.41265100 & & \\
\hline $\mathrm{H}$ & 6.42192700 & -1.59339300 & -1.26489500 & & \\
\hline 0 & 1.72522500 & -2.85046800 & 1.31438800 & & \\
\hline $\mathrm{H}$ & 0.95627200 & -2.68309600 & 0.71069100 & & \\
\hline \multicolumn{6}{|c|}{ Radical cation } \\
\hline \multicolumn{4}{|c|}{ Cartesian Coordinates } & \multicolumn{2}{|l|}{ Frequency and Energy } \\
\hline $\mathrm{O}$ & 0.17546700 & -0.32657200 & 0.57575600 & Zero-point correction $=$ & (Hartree/Particle) \\
\hline $\mathrm{O}$ & -3.55636600 & -2.55935500 & 0.18087700 & Thermal correction to Energy= & 4987 \\
\hline $\mathrm{O}$ & -0.37013700 & 2.52431400 & 0.09753600 & Thermal correction to Enthalpy= & 35932 \\
\hline $\mathrm{O}$ & -1.77452400 & -2.69640600 & 1.70207900 & Thermal correction to Gibbs Free Energy= & 0.251804 \\
\hline $\mathrm{O}$ & -4.80128600 & 1.37059800 & -0.89643400 & Sum of electronic and zero-point Energies= & -1484.498768 \\
\hline $\mathrm{O}$ & -5.39996500 & -1.40880900 & -0.51143500 & Sum of electronic and thermal Energies= & -1484.472715 \\
\hline 0 & 4.44356300 & -1.62747200 & -0.72311600 & Sum of electronic and thermal Enthalpies= & -1484.471770 \\
\hline 0 & 1.16989200 & 3.47149000 & 1.37858500 & Sum of electronic and thermal Free Energies= & -1484.555898 \\
\hline $\mathrm{O}$ & 0.77902700 & -2.63060600 & -0.79039600 & & \\
\hline C & -2.08314300 & -0.74491300 & 0.23262200 & & \\
\hline $\mathrm{C}$ & -1.01299700 & 0.17550500 & 0.27080900 & & \\
\hline $\mathrm{C}$ & -3.30673800 & -0.31751900 & -0.15956000 & & \\
\hline $\mathrm{C}$ & -2.14376900 & -2.25245400 & 0.45872600 & & \\
\hline $\mathrm{C}$ & -1.29092700 & 1.55622400 & -0.02144500 & & \\
\hline $\mathrm{C}$ & -2.55766900 & 1.99045400 & -0.42869200 & & \\
\hline $\mathrm{C}$ & -3.58966100 & 1.02833700 & -0.51242200 & & \\
\hline $\mathrm{C}$ & 1.44648700 & 0.10109600 & 0.23286000 & & \\
\hline C & 1.84684700 & 1.44250900 & 0.32800200 & & \\
\hline $\mathrm{C}$ & -4.23785700 & -1.45892900 & -0.19774600 & & \\
\hline $\mathrm{C}$ & 3.21035100 & 1.74756400 & 0.10109900 & & \\
\hline $\mathrm{C}$ & 2.31168000 & -0.94578400 & -0.06990000 & & \\
\hline $\mathrm{C}$ & -2.80629300 & 3.43773000 & -0.73885500 & & \\
\hline $\mathrm{C}$ & 0.92199800 & 2.51820500 & 0.71955400 & & \\
\hline $\mathrm{C}$ & 3.65621200 & -0.62151900 & -0.35119300 & & \\
\hline $\mathrm{C}$ & 4.07551300 & 0.71456000 & -0.25392500 & & \\
\hline $\mathrm{C}$ & 3.75891300 & 3.14633000 & 0.20978300 & & \\
\hline $\mathrm{C}$ & 1.77856400 & -2.34862800 & -0.17518600 & & \\
\hline $\mathrm{C}$ & 5.83240700 & -1.39568900 & -1.01718900 & & \\
\hline $\mathrm{H}$ & -1.53976900 & -2.79419100 & -0.26820700 & & \\
\hline $\mathrm{H}$ & -2.24280600 & 3.74428100 & -1.62465100 & & \\
\hline $\mathrm{H}$ & -3.86337000 & 3.61740100 & -0.92207000 & & \\
\hline $\mathrm{H}$ & -2.47090600 & 4.06816700 & 0.08742100 & & \\
\hline $\mathrm{H}$ & 5.10756600 & 0.96199000 & -0.45743000 & & \\
\hline $\mathrm{H}$ & 3.15303900 & 3.86687300 & -0.34316500 & & \\
\hline $\mathrm{H}$ & 3.76826500 & 3.47757200 & 1.25025900 & & \\
\hline $\mathrm{H}$ & 4.77655000 & 3.18364100 & -0.17720700 & & \\
\hline $\mathrm{H}$ & -2.31472600 & -2.27496900 & 2.38322100 & & \\
\hline $\mathrm{H}$ & -5.38938900 & 0.58116900 & -0.88744700 & & \\
\hline $\mathrm{H}$ & 6.35407600 & -1.00971300 & -0.13859300 & & \\
\hline
\end{tabular}




\begin{tabular}{|c|c|c|c|c|c|}
\hline$r$ & 5.94095700 & -0.71221800 & -1.86264300 & & \\
\hline $\mathrm{H}$ & 6.23085600 & -2.37152600 & -1.28040100 & & \\
\hline 0 & 2.52117700 & -3.22590500 & 0.49999200 & & \\
\hline $\mathrm{H}$ & 2.13536200 & -4.10716700 & 0.36931100 & & \\
\hline & ompound (7) & & & & \\
\hline & Coordinates & & & Frequency and Energy & \\
\hline $\mathrm{O}$ & 0.49168100 & 0.52186200 & -1.44669100 & Zero-point correction $=$ & (Hartree/Particle) \\
\hline 0 & -4.90467400 & -0.04454400 & 0.93954600 & Thermal correction to Energy= & 3672 \\
\hline $\mathrm{O}$ & 0.37008900 & -2.17825300 & -0.75522700 & Thermal correction to Enthalpy= & 54616 \\
\hline 0 & -1.68848700 & 2.15153700 & -0.88790500 & Thermal correction to Gibbs Free Energy= & 0.189633 \\
\hline 0 & -4.40120500 & 2.05417300 & 0.22712700 & Sum of electronic and zero-point Energies= & -1142.355592 \\
\hline 0 & 2.44722400 & -2.88453900 & -0.74007000 & Sum of electronic and thermal Energies= & -1142.337516 \\
\hline 0 & 3.88172900 & 3.12773900 & 0.66239400 & Sum of electronic and thermal Enthalpies= & -1142.336571 \\
\hline $\mathrm{C}$ & -2.87768700 & -1.09247200 & 0.37526600 & Sum of electronic and thermal Free Energies= & -1142.401555 \\
\hline $\mathrm{C}$ & -2.83279800 & 0.24846300 & 0.02592600 & & \\
\hline $\mathrm{C}$ & -0.62397100 & 0.01416100 & -0.81484900 & & \\
\hline $\mathrm{C}$ & -0.66682100 & -1.33513400 & -0.44976400 & & \\
\hline $\mathrm{C}$ & -4.23900300 & -1.33107500 & 0.97998400 & & \\
\hline $\mathrm{C}$ & -1.71961000 & 0.84847600 & -0.56428200 & & \\
\hline $\mathrm{C}$ & -1.79303300 & -1.91953100 & 0.14705500 & & \\
\hline $\mathrm{C}$ & 2.20373000 & -0.59744800 & -0.14618700 & & \\
\hline C & 1.61922800 & 0.57616100 & -0.64326400 & & \\
\hline $\mathrm{C}$ & -4.08911900 & 0.89567400 & 0.37996000 & & \\
\hline $\mathrm{C}$ & 3.37889800 & -0.47690100 & 0.64421000 & & \\
\hline $\mathrm{C}$ & 1.73054600 & -1.94147100 & -0.56221800 & & \\
\hline C & 2.14814200 & 1.83385700 & -0.40526300 & & \\
\hline C & 3.89024400 & 0.78825600 & 0.90345400 & & \\
\hline $\mathrm{C}$ & 3.29857900 & 1.93789400 & 0.37275900 & & \\
\hline $\mathrm{C}$ & 4.09576200 & -1.67289800 & 1.22200600 & & \\
\hline $\mathrm{H}$ & -4.84248100 & -2.04110400 & 0.40963300 & & \\
\hline $\mathrm{H}$ & -4.19553300 & -1.65547400 & 2.02228500 & & \\
\hline $\mathrm{H}$ & -1.77021700 & -2.97335800 & 0.39150900 & & \\
\hline $\mathrm{H}$ & 1.64615100 & 2.69848800 & -0.82346600 & & \\
\hline $\mathrm{H}$ & 4.77565400 & 0.90547300 & 1.51578300 & & \\
\hline $\mathrm{H}$ & 3.40555700 & -2.36648900 & 1.70648600 & & \\
\hline $\mathrm{H}$ & 4.60070000 & -2.23858500 & 0.43731000 & & \\
\hline $\mathrm{H}$ & 4.83449200 & -1.34787700 & 1.95587800 & & \\
\hline $\mathrm{H}$ & -2.55095800 & 2.53664200 & -0.64400900 & & \\
\hline $\mathrm{H}$ & 3.38727000 & 3.84240900 & 0.24644500 & & \\
\hline & & & & & \\
\hline & Coordinates & & & Frequency and Energy & \\
\hline $\mathrm{O}$ & 0.45162100 & 0.51526600 & -1.44147200 & Zero-point correction= & (Hartree/Particle) \\
\hline $\mathrm{O}$ & -4.89902600 & -0.16097400 & 0.97673600 & Thermal correction to Energy= & 0031 \\
\hline $\mathrm{O}$ & 0.35667200 & -2.18239200 & -0.76127800 & Thermal correction to Enthalpy= & 40976 \\
\hline $\mathrm{O}$ & -1.71998500 & 2.13666300 & -0.89861100 & Thermal correction to Gibbs Free Energy= & 0.175594 \\
\hline $\mathrm{O}$ & -4.51579100 & 1.99483600 & 0.27369400 & Sum of electronic and zero-point Energies= & -1141.725042 \\
\hline $\mathrm{O}$ & 2.43667500 & -2.87926500 & -0.72715100 & Sum of electronic and thermal Energies= & -1141.707030 \\
\hline $\mathrm{O}$ & 3.83420400 & 3.14721900 & 0.64590600 & Sum of electronic and thermal Enthalpies= & -1141.706086 \\
\hline $\mathrm{C}$ & -2.90564400 & -1.12170300 & 0.40456800 & Sum of electronic and thermal Free Energies= & -1141.771467 \\
\hline $\mathrm{C}$ & -2.86643100 & 0.24506200 & 0.03801300 & & \\
\hline $\mathrm{C}$ & -0.65809100 & 0.00396500 & -0.80881500 & & \\
\hline $\mathrm{C}$ & -0.68845800 & -1.35360200 & -0.43694100 & & \\
\hline C & -4.17548900 & -1.31595800 & 0.97368600 & & \\
\hline C & -1.75842000 & 0.83683000 & -0.56059300 & & \\
\hline
\end{tabular}




\begin{tabular}{|c|c|c|c|c|c|}
\hline & & & & \\
\hline $\mathrm{C}$ & -1.79314200 & -1.94529200 & 0.16493100 & & \\
\hline C & 2.17690700 & -0.59193300 & -0.14317000 & & \\
\hline C & 1.58448700 & 0.57616700 & -0.64280800 & & \\
\hline C & -4.11736800 & 0.86061400 & 0.39657000 & & \\
\hline C & 3.35275000 & -0.46035100 & 0.64470400 & & \\
\hline C & 1.71269200 & -1.94049100 & -0.55541000 & & \\
\hline $\mathrm{C}$ & 2.10582500 & 1.83820400 & -0.41184500 & & \\
\hline $\mathrm{C}$ & 3.85688500 & 0.80897400 & 0.89747500 & & \\
\hline $\mathrm{C}$ & 3.25753200 & 1.95273900 & 0.36291300 & & \\
\hline $\mathrm{C}$ & 4.07797000 & -1.64927800 & 1.22650000 & & \\
\hline $\mathrm{H}$ & -4.65635200 & -2.18429000 & 1.39256900 & & \\
\hline $\mathrm{H}$ & -1.76453000 & -2.99814300 & 0.41041100 & & \\
\hline $\mathrm{H}$ & 1.59743700 & 2.69796400 & -0.83230300 & & \\
\hline $\mathrm{H}$ & 4.74271500 & 0.93412000 & 1.50761400 & & \\
\hline $\mathrm{H}$ & 3.39276800 & -2.34512900 & 1.71474300 & & \\
\hline $\mathrm{H}$ & 4.58520000 & -2.21507100 & 0.44338500 & & \\
\hline $\mathrm{H}$ & 4.81565600 & -1.31643800 & 1.95790700 & & \\
\hline $\mathrm{H}$ & -2.57529500 & 2.53526700 & -0.66277300 & & \\
\hline $\mathrm{H}$ & 3.33540600 & 3.85722600 & 0.22718700 & & \\
\hline \multicolumn{6}{|c|}{ Anion } \\
\hline \multicolumn{4}{|c|}{ Cartesian Coordinates } & \multicolumn{2}{|l|}{ Frequency and Energy } \\
\hline $\mathrm{O}$ & 0.48892100 & 0.63945000 & -1.36980600 & Zero-point correction $=$ & (Hartree/Particle) \\
\hline 0 & -4.94044300 & -0.11541800 & 0.89409200 & Thermal correction to Energy= & 9729 \\
\hline $\mathrm{O}$ & 0.36898000 & -2.07217900 & -0.88183900 & Thermal correction to Enthalpy= & 0673 \\
\hline $\mathrm{O}$ & -1.70700100 & 2.23265000 & -0.72588600 & Thermal correction to Gibbs Free Energy= & 0.175783 \\
\hline $\mathrm{O}$ & -4.45727900 & 2.03346700 & 0.34441500 & Sum of electronic and zero-point Energies= & -1141.834353 \\
\hline $\mathrm{O}$ & 2.36271400 & -2.94039500 & -0.49652300 & Sum of electronic and thermal Energies= & -1141.816492 \\
\hline 0 & 3.99232100 & 3.13859600 & 0.55886200 & Sum of electronic and thermal Enthalpies= & -1141.815548 \\
\hline C & -2.89312200 & -1.10125800 & 0.28910400 & Sum of electronic and thermal Free Energies= & -1141.880437 \\
\hline $\mathrm{C}$ & -2.85016900 & 0.26701000 & 0.04527300 & & \\
\hline $\mathrm{C}$ & -0.61462000 & 0.10322800 & -0.77617800 & & \\
\hline $\mathrm{C}$ & -0.66231800 & -1.27706700 & -0.52552900 & & \\
\hline $\mathrm{C}$ & -4.25934300 & -1.39307100 & 0.85487300 & & \\
\hline $\mathrm{C}$ & -1.72286700 & 0.90766900 & -0.48292000 & & \\
\hline C & -1.80519000 & -1.90226500 & 0.01155800 & & \\
\hline $\mathrm{C}$ & 2.23835200 & -0.58444400 & -0.13660500 & & \\
\hline $\mathrm{C}$ & 1.66402900 & 0.64467400 & -0.58854200 & & \\
\hline $\mathrm{C}$ & -4.11360800 & 0.87261200 & 0.41595700 & & \\
\hline $\mathrm{C}$ & 3.48113100 & -0.45533100 & 0.59559300 & & \\
\hline $\mathrm{C}$ & 1.76198000 & -1.89209900 & -0.48906600 & & \\
\hline $\mathrm{C}$ & 2.21593700 & 1.87092900 & -0.37577200 & & \\
\hline $\mathrm{C}$ & 4.02572800 & 0.77963900 & 0.82953700 & & \\
\hline $\mathrm{C}$ & 3.46124000 & 2.03088300 & 0.35221200 & & \\
\hline $\mathrm{C}$ & 4.19831900 & -1.66533000 & 1.15583500 & & \\
\hline $\mathrm{H}$ & -4.84888600 & -2.06987700 & 0.23001100 & & \\
\hline $\mathrm{H}$ & -4.22776200 & -1.78928500 & 1.87364200 & & \\
\hline $\mathrm{H}$ & -1.78126000 & -2.97239000 & 0.17400400 & & \\
\hline $\mathrm{H}$ & 1.72006500 & 2.75212700 & -0.76376000 & & \\
\hline $\mathrm{H}$ & 4.94469000 & 0.86624500 & 1.40211800 & & \\
\hline $\mathrm{H}$ & 3.52637600 & -2.30833700 & 1.73070200 & & \\
\hline $\mathrm{H}$ & 4.60638000 & -2.29336400 & 0.36147300 & & \\
\hline $\mathrm{H}$ & 5.01241500 & -1.33737200 & 1.80665600 & & \\
\hline $\mathrm{H}$ & -2.57060000 & 2.58820900 & -0.45336000 & & \\
\hline \multicolumn{4}{|c|}{ Radical cation } & & \\
\hline
\end{tabular}




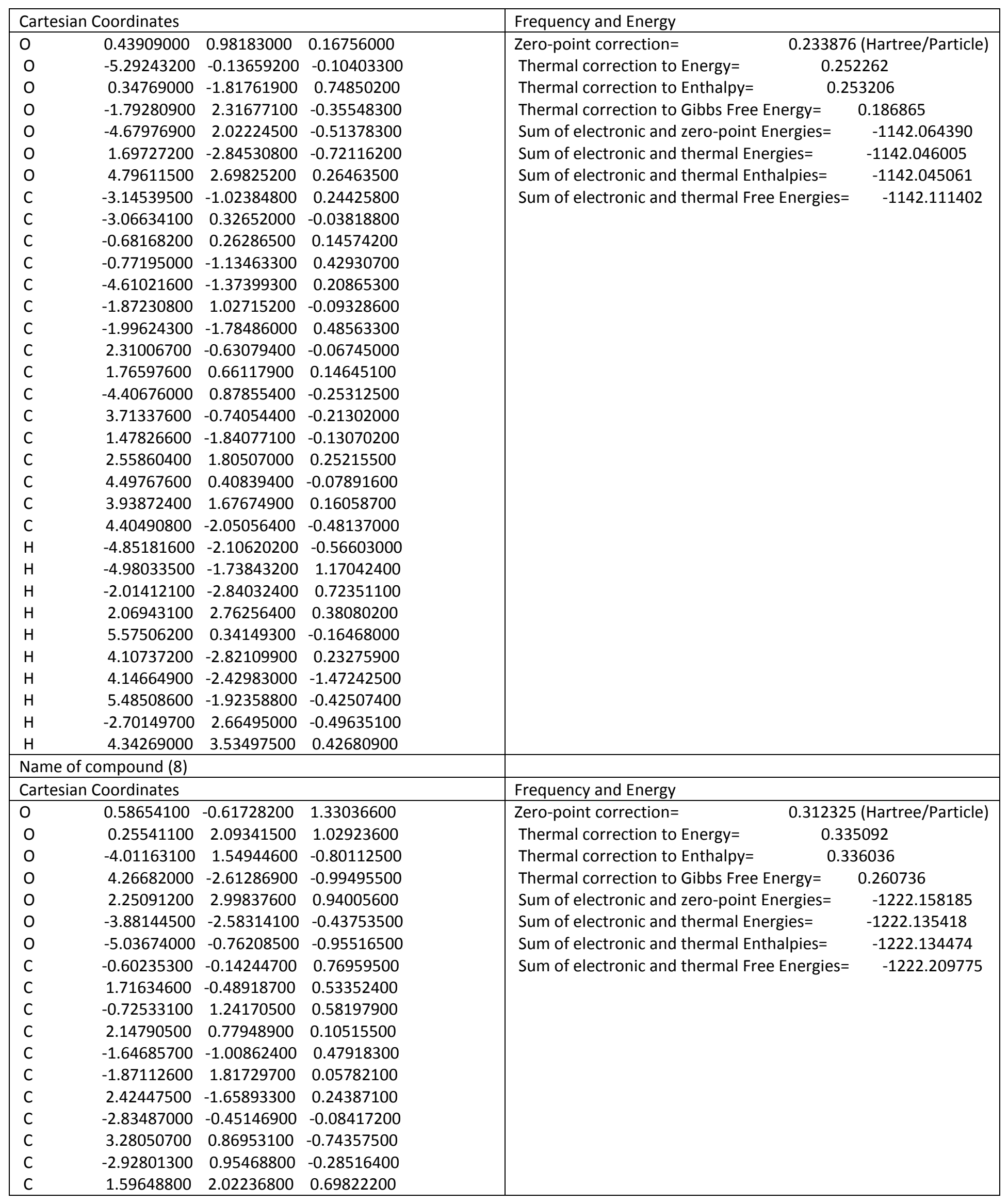




\begin{tabular}{|c|c|c|c|c|c|}
\hline \multirow{2}{*}{\multicolumn{3}{|c|}{$352984200-153685100-060975300$}} & & & \\
\hline & 3.52984200 & -1.53685100 & -0.60975300 & & \\
\hline C & 3.92654100 & -0.29928200 & -1.11459700 & & \\
\hline $\mathrm{C}$ & -1.49851200 & -2.46906300 & 0.83083900 & & \\
\hline C & -2.02551300 & 3.30072500 & -0.13569800 & & \\
\hline C & 2.05704200 & -2.99788100 & 0.83328300 & & \\
\hline C & 3.81058000 & 2.18243400 & -1.26746600 & & \\
\hline C & -3.99419400 & -1.24334200 & -0.51517600 & & \\
\hline $\mathrm{H}$ & 4.77866800 & -0.27103000 & -1.78233600 & & \\
\hline $\mathrm{H}$ & -2.36630900 & -2.82824800 & 1.38207700 & & \\
\hline $\mathrm{H}$ & -1.41325200 & -3.09237300 & -0.06246800 & & \\
\hline $\mathrm{H}$ & -0.61515200 & -2.60902400 & 1.44415600 & & \\
\hline $\mathrm{H}$ & -2.90115000 & 3.66735600 & 0.40610400 & & \\
\hline $\mathrm{H}$ & -1.14412400 & 3.83327300 & 0.21424100 & & \\
\hline $\mathrm{H}$ & -2.19112200 & 3.53449200 & -1.19115400 & & \\
\hline $\mathrm{H}$ & 1.46938600 & -3.61587400 & 0.14546400 & & \\
\hline $\mathrm{H}$ & 2.95431100 & -3.56288000 & 1.11100800 & & \\
\hline $\mathrm{H}$ & 1.47727700 & -2.87107700 & 1.74441500 & & \\
\hline $\mathrm{H}$ & 3.01087700 & 2.83717000 & -1.61832500 & & \\
\hline $\mathrm{H}$ & 4.33629800 & 2.72863200 & -0.48244500 & & \\
\hline $\mathrm{H}$ & 4.49987000 & 2.00148100 & -2.09378900 & & \\
\hline $\mathrm{H}$ & -4.67986800 & 0.84597900 & -0.97820800 & & \\
\hline $\mathrm{H}$ & 3.89056600 & -3.41833500 & -0.62558100 & & \\
\hline $\mathrm{H}$ & -4.72295800 & -2.93336800 & -0.76740300 & & \\
\hline \multicolumn{6}{|c|}{ Radical } \\
\hline \multicolumn{4}{|c|}{ Cartesian Coordinates } & \multicolumn{2}{|l|}{ Frequency and Energy } \\
\hline $\mathrm{O}$ & 0.59714100 & -0.65029500 & 1.33621700 & Zero-point correction $=$ & (Hartree/Particle) \\
\hline 0 & 0.34636900 & 2.08213700 & 0.99586400 & Thermal correction to Energy= & 1536 \\
\hline 0 & -3.91656700 & 1.62519000 & -0.86258400 & Thermal correction to Enthalpy= & 2481 \\
\hline 0 & 4.15553100 & -2.68637800 & -1.04807300 & Thermal correction to Gibbs Free Energy $=$ & 0.246746 \\
\hline $\mathrm{O}$ & 2.41949900 & 2.77454800 & 1.20482100 & Sum of electronic and zero-point Energies= & -1221.527063 \\
\hline 0 & -3.94786100 & -2.49257100 & -0.35016800 & Sum of electronic and thermal Energies= & -1221.504493 \\
\hline 0 & -5.02790300 & -0.64816400 & -0.94427900 & Sum of electronic and thermal Enthalpies= & -1221.503549 \\
\hline $\mathrm{C}$ & -0.57686200 & -0.14130200 & 0.77209600 & Sum of electronic and thermal Free Energies= & -1221.579283 \\
\hline $\mathrm{C}$ & 1.71724300 & -0.58446700 & 0.51650100 & & \\
\hline $\mathrm{C}$ & -0.65428800 & 1.24203900 & 0.55112000 & & \\
\hline c & 2.20105900 & 0.68487300 & 0.12379900 & & \\
\hline c & -1.65394500 & -0.97631000 & 0.50774100 & & \\
\hline C & -1.77537000 & 1.84383200 & 0.00592500 & & \\
\hline C & 2.36997200 & -1.75635700 & 0.18511900 & & \\
\hline $\mathrm{C}$ & -2.81862500 & -0.39305600 & -0.07485800 & & \\
\hline C & 3.32304300 & 0.79976800 & -0.76250100 & & \\
\hline $\mathrm{C}$ & -2.85931700 & 1.00784300 & -0.32171100 & & \\
\hline $\mathrm{C}$ & 1.69041500 & 1.91106700 & 0.80304100 & & \\
\hline $\mathrm{C}$ & 3.53422500 & -1.66368600 & -0.70203500 & & \\
\hline $\mathrm{C}$ & 3.93235900 & -0.35333800 & -1.17643600 & & \\
\hline $\mathrm{C}$ & -1.55820700 & -2.42789200 & 0.91145900 & & \\
\hline $\mathrm{C}$ & -1.88261500 & 3.32617000 & -0.22450300 & & \\
\hline $\mathrm{C}$ & 1.96051100 & -3.10627600 & 0.68183500 & & \\
\hline $\mathrm{C}$ & 3.82490400 & 2.13917800 & -1.24393500 & & \\
\hline $\mathrm{C}$ & -4.00861800 & -1.15552900 & -0.48140200 & & \\
\hline $\mathrm{H}$ & 4.77427100 & -0.33096300 & -1.85809700 & & \\
\hline $\mathrm{H}$ & -2.43382000 & -2.72881900 & 1.48505700 & & \\
\hline $\mathrm{H}$ & -1.51158200 & -3.08627700 & 0.04111800 & & \\
\hline $\mathrm{H}$ & -0.67282400 & -2.58195000 & 1.51955000 & & \\
\hline
\end{tabular}




\begin{tabular}{|c|c|c|c|c|c|}
\hline & & & & \\
\hline $\mathrm{H}$ & -2.75778300 & 3.72918400 & 0.29111700 & & \\
\hline $\mathrm{H}$ & -0.99459300 & 3.84444500 & 0.12994600 & & \\
\hline $\mathrm{H}$ & -2.02156200 & 3.53927500 & -1.28815400 & & \\
\hline $\mathrm{H}$ & 2.80210900 & -3.79164000 & 0.59201000 & & \\
\hline $\mathrm{H}$ & 1.62018700 & -3.06212000 & 1.71697200 & & \\
\hline $\mathrm{H}$ & 1.14118600 & -3.51441100 & 0.08054100 & & \\
\hline $\mathrm{H}$ & 3.01040900 & 2.76714000 & -1.61355700 & & \\
\hline $\mathrm{H}$ & 4.30633100 & 2.68868000 & -0.43402100 & & \\
\hline $\mathrm{H}$ & 4.54267600 & 1.99766600 & -2.05259700 & & \\
\hline $\mathrm{H}$ & -4.61026400 & 0.94150800 & -1.02141300 & & \\
\hline $\mathrm{H}$ & -4.79971200 & -2.82678800 & -0.67038000 & & \\
\hline \multicolumn{6}{|c|}{ ( } \\
\hline \multicolumn{4}{|c|}{ Cartesian Coordinates } & \multicolumn{2}{|l|}{ Frequency and Energy } \\
\hline 0 & -0.59178000 & -0.61372700 & -1.37931300 & Zero-point correction $=$ & (Hartree/Particle) \\
\hline 0 & -0.25562700 & 2.10513500 & -1.05899100 & Thermal correction to Energy= & 198 \\
\hline 0 & 3.99751900 & 1.46539700 & 0.87705000 & Thermal correction to Enthalpy= & 1143 \\
\hline $\mathrm{O}$ & -4.18500100 & -2.62625100 & 1.06640700 & Thermal correction to Gibbs Free Energy $=$ & 0.245611 \\
\hline $\mathrm{O}$ & -2.27069600 & 2.96653600 & -1.02076900 & Sum of electronic and zero-point Energies= & -1221.644900 \\
\hline 0 & 3.96490100 & -2.59036600 & 0.38390800 & Sum of electronic and thermal Energies= & -1221.622177 \\
\hline 0 & 5.02485100 & -0.74548600 & 1.01650600 & Sum of electronic and thermal Enthalpies= & -1221.621233 \\
\hline $\mathrm{C}$ & 0.62217900 & -0.13992600 & -0.81411900 & Sum of electronic and thermal Free Energies= & -1221.696764 \\
\hline $\mathrm{C}$ & -1.68757800 & -0.49151500 & -0.55891900 & & \\
\hline $\mathrm{C}$ & 0.74179300 & 1.23658900 & -0.59305300 & & \\
\hline C & -2.11919000 & 0.77682900 & -0.12798300 & & \\
\hline $\mathrm{C}$ & 1.66298200 & -1.01041300 & -0.50660700 & & \\
\hline $\mathrm{C}$ & 1.86525200 & 1.79954900 & -0.03159000 & & \\
\hline $\mathrm{C}$ & -2.38651800 & -1.66155600 & -0.22903700 & & \\
\hline $\mathrm{C}$ & 2.83407200 & -0.47988700 & 0.09022100 & & \\
\hline $\mathrm{C}$ & -3.22778100 & 0.86934200 & 0.74564900 & & \\
\hline $\mathrm{C}$ & 2.95259700 & 0.93762800 & 0.34357500 & & \\
\hline $\mathrm{C}$ & -1.57073900 & 2.01935500 & -0.74731200 & & \\
\hline $\mathrm{C}$ & -3.46775900 & -1.53725600 & 0.64848900 & & \\
\hline $\mathrm{C}$ & -3.86385500 & -0.29756000 & 1.14645800 & & \\
\hline $\mathrm{C}$ & 1.52299700 & -2.47212100 & -0.86809600 & & \\
\hline $\mathrm{C}$ & 2.02626100 & 3.27559500 & 0.19653100 & & \\
\hline $\mathrm{C}$ & -2.00328200 & -3.00640500 & -0.79349800 & & \\
\hline $\mathrm{C}$ & -3.74362300 & 2.18828100 & 1.27204400 & & \\
\hline $\mathrm{C}$ & 3.97147900 & -1.37223400 & 0.49658100 & & \\
\hline $\mathrm{H}$ & -4.69713200 & -0.26467500 & 1.83824800 & & \\
\hline $\mathrm{H}$ & 2.44527600 & -2.84559000 & -1.30656500 & & \\
\hline $\mathrm{H}$ & 1.35241900 & -3.08880900 & 0.01951400 & & \\
\hline $\mathrm{H}$ & 0.69829900 & -2.60937900 & -1.56447900 & & \\
\hline $\mathrm{H}$ & 1.17897200 & 3.83971200 & -0.19287400 & & \\
\hline $\mathrm{H}$ & 2.14068200 & 3.48671300 & 1.26548600 & & \\
\hline $\mathrm{H}$ & 2.94789900 & 3.62933100 & -0.27566900 & & \\
\hline $\mathrm{H}$ & -1.39584300 & -3.59562100 & -0.09749900 & & \\
\hline $\mathrm{H}$ & -2.89214900 & -3.59416000 & -1.05545100 & & \\
\hline $\mathrm{H}$ & -1.42019600 & -2.88226600 & -1.70243700 & & \\
\hline $\mathrm{H}$ & -2.93254200 & 2.83287900 & 1.61676700 & & \\
\hline $\mathrm{H}$ & -4.26520200 & 2.74115700 & 0.48917100 & & \\
\hline $\mathrm{H}$ & -4.42855800 & 2.01519700 & 2.10523600 & & \\
\hline $\mathrm{H}$ & 4.78694700 & 0.26289800 & 1.04231600 & & \\
\hline $\mathrm{H}$ & -3.76794000 & -3.42306400 & 0.72383400 & & \\
\hline & & & & & \\
\hline
\end{tabular}




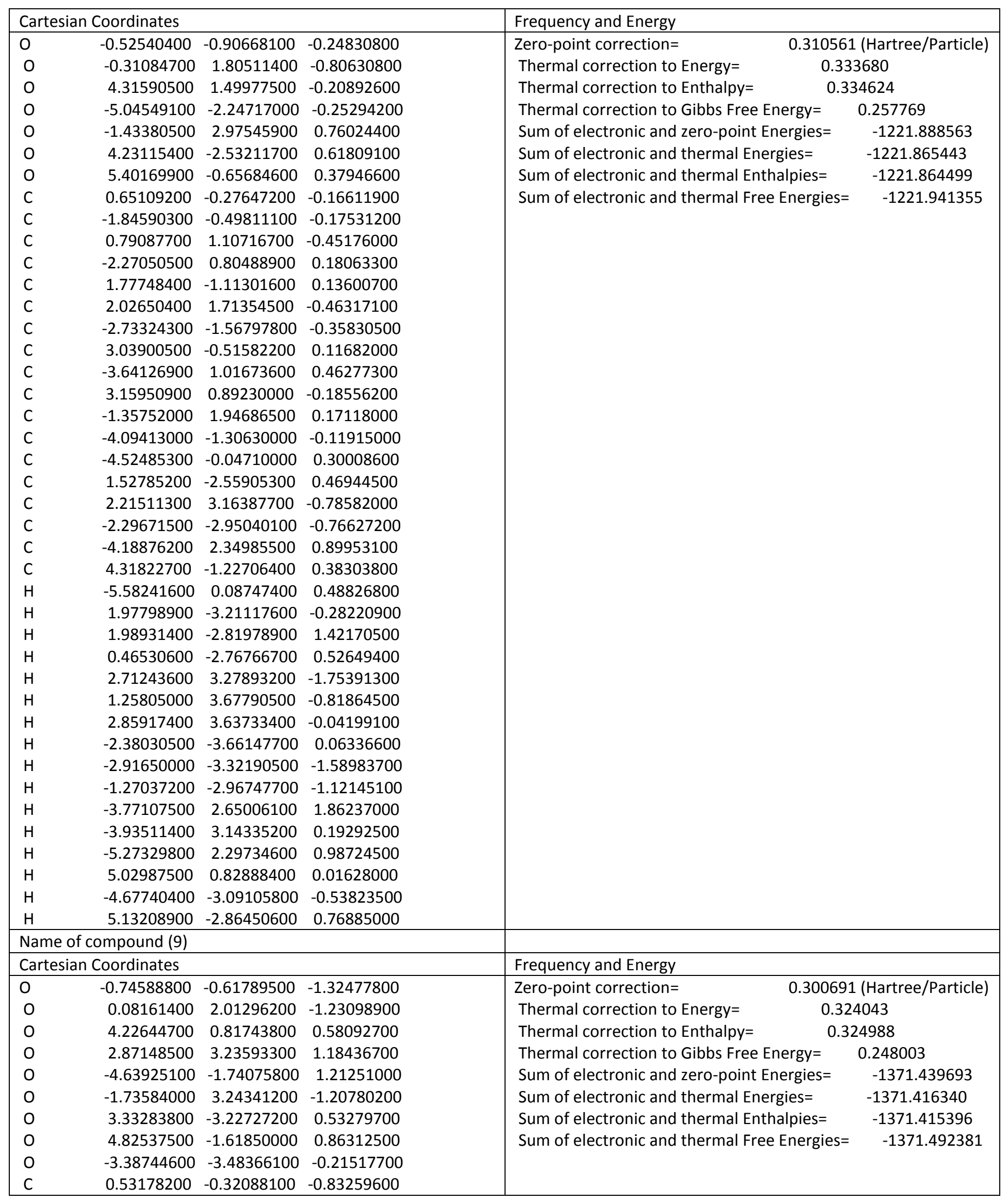




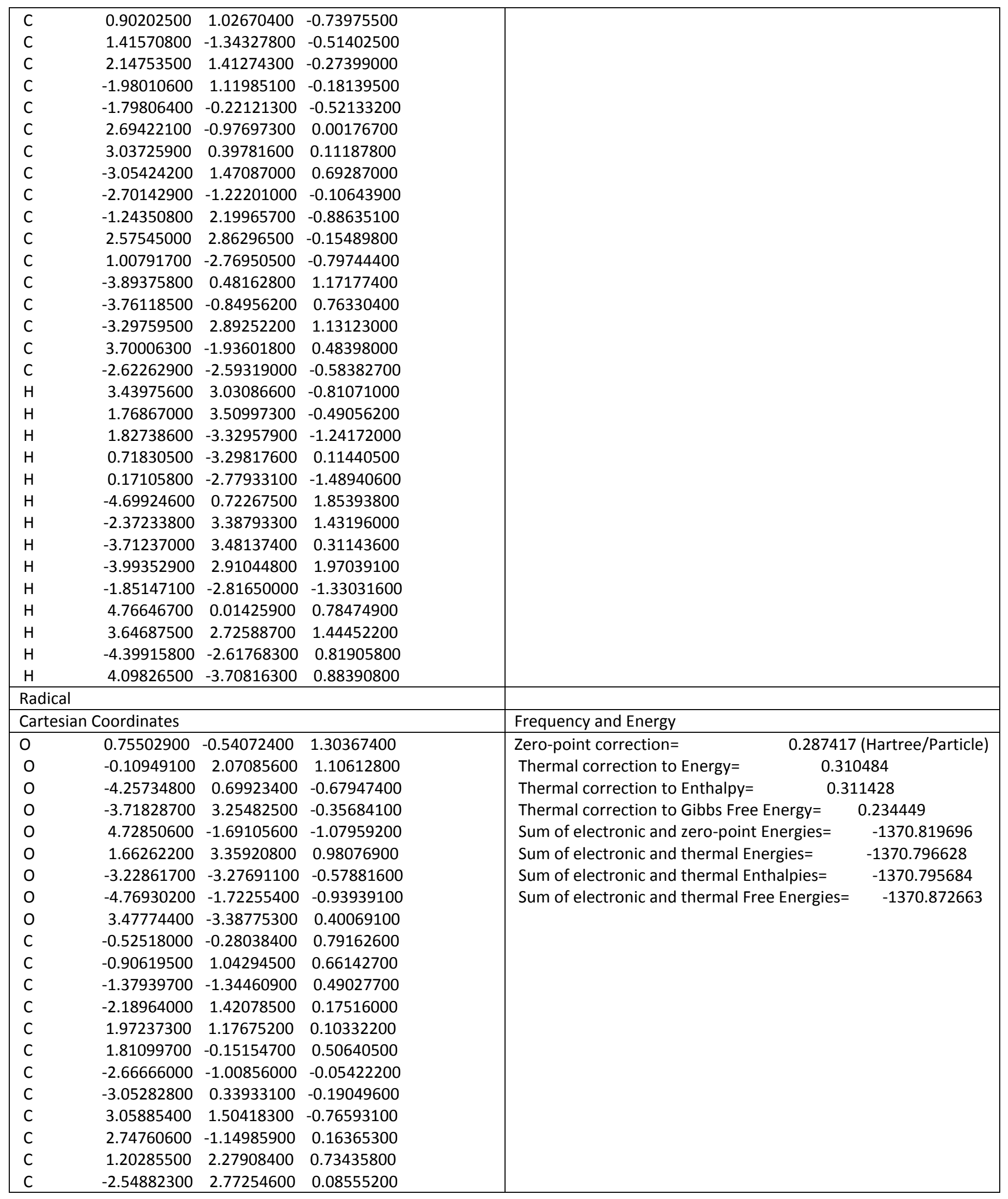




\begin{tabular}{|c|c|c|c|c|c|}
\hline C & -0.95496900 & -2.75119600 & 0.82980800 & & \\
\hline C & 3.93181800 & 0.51187900 & -1.17339700 & & \\
\hline C & 3.82012200 & -0.79855300 & -0.69825500 & & \\
\hline C & 3.28581000 & 2.90474700 & -1.27617100 & & \\
\hline C & -3.63575700 & -1.99950000 & -0.54211800 & & \\
\hline C & 2.68280500 & -2.49852300 & 0.70359300 & & \\
\hline $\mathrm{H}$ & -1.85878800 & 3.54170600 & 0.39898500 & & \\
\hline $\mathrm{H}$ & -1.77447800 & -3.31479500 & 1.27198800 & & \\
\hline $\mathrm{H}$ & -0.62518600 & -3.31100400 & -0.05074200 & & \\
\hline $\mathrm{H}$ & -0.13937200 & -2.72278800 & 1.54717100 & & \\
\hline $\mathrm{H}$ & 4.74747100 & 0.73639400 & -1.84911000 & & \\
\hline $\mathrm{H}$ & 2.35839400 & 3.36950100 & -1.61610500 & & \\
\hline $\mathrm{H}$ & 3.67852700 & 3.54357300 & -0.48340100 & & \\
\hline $\mathrm{H}$ & 3.99492500 & 2.88919200 & -2.10451200 & & \\
\hline $\mathrm{H}$ & 1.89317900 & -2.70373200 & 1.43539000 & & \\
\hline $\mathrm{H}$ & -4.76252200 & -0.13683900 & -0.87763800 & & \\
\hline $\mathrm{H}$ & -4.28568500 & 2.50754500 & -0.61312700 & & \\
\hline $\mathrm{H}$ & 4.49689600 & -2.55178300 & -0.64739700 & & \\
\hline $\mathrm{H}$ & -3.97295600 & -3.78508100 & -0.93621800 & & \\
\hline \multicolumn{6}{|c|}{ 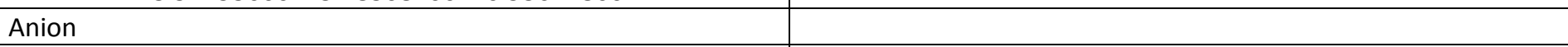 } \\
\hline \multicolumn{4}{|c|}{ Cartesian Coordinates } & \multicolumn{2}{|l|}{ Frequency and Energy } \\
\hline 0 & -0.76687200 & -0.62413800 & -1.31971100 & Zero-point correction= & (Hartree/Particle) \\
\hline $\mathrm{O}$ & 0.11174900 & 2.00315200 & -1.25481300 & Thermal correction to Energy= & 9875 \\
\hline $\mathrm{O}$ & 4.23470200 & 0.68509800 & 0.62023900 & Thermal correction to Enthalpy= & 0819 \\
\hline $\mathrm{O}$ & 3.19046000 & 3.12681500 & 1.08137700 & Thermal correction to Gibbs Free Energy= & 0.234528 \\
\hline $\mathrm{O}$ & -4.70560600 & -1.64310300 & 1.18167900 & Sum of electronic and zero-point Energies= & -1370.941441 \\
\hline $\mathrm{O}$ & -1.68644700 & 3.25692500 & -1.19868600 & Sum of electronic and thermal Energies= & -1370.918486 \\
\hline O & 3.38105000 & -3.31125300 & 0.48348000 & Sum of electronic and thermal Enthalpies= & -1370.917542 \\
\hline O & 4.81836800 & -1.68131500 & 0.91794800 & Sum of electronic and thermal Free Energies= & -1370.993833 \\
\hline 0 & -3.49805100 & -3.41020700 & -0.24256600 & & \\
\hline $\mathrm{C}$ & 0.54100000 & -0.34303200 & -0.83347900 & & \\
\hline $\mathrm{C}$ & 0.93424000 & 0.99345000 & -0.74002600 & & \\
\hline C & 1.40308400 & -1.37945200 & -0.48742200 & & \\
\hline C & 2.17174000 & 1.35968500 & -0.26340400 & & \\
\hline C & -1.93081300 & 1.14292800 & -0.16317300 & & \\
\hline C & -1.78868200 & -0.20288400 & -0.51880800 & & \\
\hline C & 2.67970100 & -1.04801700 & 0.03184500 & & \\
\hline C & 3.07734200 & 0.33135300 & 0.15560000 & & \\
\hline C & -2.98305800 & 1.51441700 & 0.72097400 & & \\
\hline C & -2.73719300 & -1.17240400 & -0.11143200 & & \\
\hline C & -1.17415200 & 2.21046300 & -0.88314700 & & \\
\hline C & 2.65039400 & 2.79400600 & -0.19163400 & & \\
\hline C & 0.95590700 & -2.80426000 & -0.72875200 & & \\
\hline C & -3.86594000 & 0.55285900 & 1.18725300 & & \\
\hline C & -3.78724500 & -0.77210000 & 0.75425300 & & \\
\hline C & -3.16813100 & 2.93703400 & 1.18790700 & & \\
\hline C & 3.63601800 & -2.11773300 & 0.48566300 & & \\
\hline C & -2.68061900 & -2.54558200 & -0.57448100 & & \\
\hline $\mathrm{H}$ & 3.40722300 & 2.94722100 & -0.97891400 & & \\
\hline $\mathrm{H}$ & 1.82789000 & 3.48139500 & -0.38879000 & & \\
\hline $\mathrm{H}$ & 1.79118000 & -3.41642100 & -1.05493900 & & \\
\hline $\mathrm{H}$ & 0.58686100 & -3.27011100 & 0.19055400 & & \\
\hline $\mathrm{H}$ & 0.16521900 & -2.82620200 & -1.47773500 & & \\
\hline $\mathrm{H}$ & -4.65862300 & 0.81673800 & 1.87654400 & & \\
\hline
\end{tabular}




\begin{tabular}{|c|c|c|c|c|c|}
\hline $\mathrm{H}$ & -2.21984400 & 3.38875900 & 1.48556700 & & \\
\hline $\mathrm{H}$ & -3.56515000 & 3.55617900 & 0.38211100 & & \\
\hline $\mathrm{H}$ & -3.85360800 & 2.96646400 & 2.03674600 & & \\
\hline $\mathrm{H}$ & -1.87106400 & -2.80082400 & -1.26652000 & & \\
\hline $\mathrm{H}$ & 4.79876700 & -0.65904200 & 0.87041700 & & \\
\hline $\mathrm{H}$ & 3.83497700 & 2.41258300 & 1.23729200 & & \\
\hline $\mathrm{H}$ & -4.48019400 & -2.51691600 & 0.76965500 & & \\
\hline \multicolumn{6}{|c|}{ Radical cation } \\
\hline \multicolumn{4}{|c|}{ Cartesian Coordinates } & \multicolumn{2}{|l|}{ Frequency and Energy } \\
\hline $\mathrm{O}$ & -0.66154600 & -0.82642000 & 0.10290700 & Zero-point correction $=$ & 0.298079 (Hartree/Particle) \\
\hline $\mathrm{O}$ & -0.08769800 & 1.86252100 & 0.54559300 & Thermal correction to Energy= & 0.322114 \\
\hline $\mathrm{O}$ & 4.45966800 & 0.90339700 & 0.02642100 & Thermal correction to Enthalpy= & 0.323058 \\
\hline 0 & 3.29649000 & 2.90393800 & 1.82720800 & Thermal correction to Gibbs Free Energy= & 0.243567 \\
\hline 0 & -5.30230000 & -1.57387200 & 0.39106600 & Sum of electronic and zero-point Energies= & -1371.154244 \\
\hline 0 & -1.07859000 & 3.03834200 & -1.10549900 & Sum of electronic and thermal Energies= & -1371.130209 \\
\hline $\mathrm{O}$ & 3.83737600 & -3.10499600 & -0.63521000 & Sum of electronic and thermal Enthalpies= & -1371.129265 \\
\hline $\mathrm{O}$ & 5.24403500 & -1.38748300 & -0.49560300 & \multirow{34}{*}{\multicolumn{2}{|c|}{ Sum of electronic and thermal Free Energies= }} \\
\hline 0 & -3.55606100 & -3.36584000 & 1.01241800 & & \\
\hline $\mathrm{C}$ & 0.58794600 & -0.35528200 & -0.00312600 & & \\
\hline $\mathrm{C}$ & 0.91124800 & 1.00813900 & 0.23830400 & & \\
\hline $\mathrm{C}$ & 1.59080100 & -1.34297000 & -0.28584200 & & \\
\hline $\mathrm{C}$ & 2.21737700 & 1.43584700 & 0.24380400 & & \\
\hline $\mathrm{C}$ & -2.19369300 & 1.07028400 & -0.30673500 & & \\
\hline $\mathrm{C}$ & -1.91826200 & -0.25329200 & 0.07318100 & & \\
\hline C & 2.92113000 & -0.92464100 & -0.26144900 & & \\
\hline C & 3.22884600 & 0.46198800 & 0.01030100 & & \\
\hline $\mathrm{C}$ & -3.55109400 & 1.45214700 & -0.52762700 & & \\
\hline $\mathrm{C}$ & -2.93582100 & -1.19272900 & 0.33252700 & & \\
\hline $\mathrm{C}$ & -1.12939700 & 2.07008000 & -0.42195000 & & \\
\hline $\mathrm{C}$ & 2.61677100 & 2.86014600 & 0.58576100 & & \\
\hline $\mathrm{C}$ & 1.14454300 & -2.74432000 & -0.60395100 & & \\
\hline $\mathrm{C}$ & -4.55663400 & 0.53155000 & -0.28281400 & & \\
\hline $\mathrm{C}$ & -4.28121600 & -0.77375700 & 0.16188500 & & \\
\hline $\mathrm{C}$ & -3.92513300 & 2.83407700 & -0.99016200 & & \\
\hline $\mathrm{C}$ & 4.09614500 & -1.81349700 & -0.47846800 & & \\
\hline $\mathrm{C}$ & -2.66507800 & -2.55947600 & 0.78870600 & & \\
\hline $\mathrm{H}$ & 3.21868100 & 3.27015500 & -0.23185200 & & \\
\hline $\mathrm{H}$ & 1.71788900 & 3.46445400 & 0.68330800 & & \\
\hline $\mathrm{H}$ & 1.64435600 & -3.11647300 & -1.49649900 & & \\
\hline $\mathrm{H}$ & 1.40823000 & -3.42660200 & 0.20858400 & & \\
\hline $\mathrm{H}$ & 0.07193000 & -2.78600400 & -0.76135500 & & \\
\hline $\mathrm{H}$ & -5.59578300 & 0.80262600 & -0.41967700 & & \\
\hline $\mathrm{H}$ & -3.51768200 & 3.60350000 & -0.33011900 & & \\
\hline $\mathrm{H}$ & -3.52377700 & 3.03524700 & -1.98536200 & & \\
\hline $\mathrm{H}$ & -5.00858300 & 2.93951400 & -1.01842700 & & \\
\hline $\mathrm{H}$ & -1.61674100 & -2.84530400 & 0.94759200 & & \\
\hline $\mathrm{H}$ & 5.07450800 & 0.13168600 & -0.18341500 & & \\
\hline $\mathrm{H}$ & 4.23615500 & 2.76632900 & 1.67126200 & & \\
\hline $\mathrm{H}$ & -4.95532300 & -2.45411400 & 0.68290900 & & \\
\hline $\mathrm{H}$ & 4.68426400 & -3.56748700 & -0.75543400 & & \\
\hline \multicolumn{6}{|c|}{ Name of compound (10) } \\
\hline \multicolumn{4}{|c|}{ Cartesian Coordinates } & \multicolumn{2}{|l|}{ Frequency and Energy } \\
\hline 0 & -0.76909300 & -0.80775700 & -1.32602000 & Zero-point correction= & \multirow{2}{*}{$\begin{array}{c}0.318311 \text { (Hartree/Particle) } \\
0.341757\end{array}$} \\
\hline 0 & -0.22803100 & 1.89239000 & -1.20038000 & Thermal correction to Energy= & \\
\hline
\end{tabular}




\begin{tabular}{|c|c|c|c|c|c|}
\hline 0 & 4.01934800 & 1.13138900 & 0.60229600 & \multicolumn{2}{|l|}{ Thermal correction to Enthalpy= } \\
\hline 0 & 2.42472900 & 3.38573300 & 1.22673600 & Thermal correction to Gibbs Free Energy= & 0.266026 \\
\hline $\mathrm{O}$ & -4.46766000 & -2.43710000 & 1.12947300 & Sum of electronic and zero-point Energies= & -1297.385372 \\
\hline 0 & -2.16200500 & 2.92555800 & -1.21358000 & Sum of electronic and thermal Energies= & -1297.361925 \\
\hline 0 & 3.60131900 & -2.98909700 & 0.43493500 & Sum of electronic and thermal Enthalpies= & -1297.360981 \\
\hline 0 & 4.88221600 & -1.22618700 & 0.84792500 & Sum of electronic and thermal Free Energies= & -1297.437656 \\
\hline C & 0.46039200 & -0.38659400 & -0.81971900 & & \\
\hline $\mathrm{C}$ & 0.68717900 & 0.99277100 & -0.71373500 & & \\
\hline C & 1.45121900 & -1.30913700 & -0.50771800 & & \\
\hline C & 1.88462700 & 1.50620000 & -0.24252700 & & \\
\hline C & -2.20897300 & 0.78212200 & -0.19554500 & & \\
\hline C & -1.87112600 & -0.54627400 & -0.52084600 & & \\
\hline C & 2.68895700 & -0.81209200 & 0.00144200 & & \\
\hline $\mathrm{C}$ & 2.87992100 & 0.59039700 & 0.13006300 & & \\
\hline $\mathrm{C}$ & -3.31878400 & 1.02178000 & 0.64803200 & & \\
\hline $\mathrm{C}$ & -2.64223300 & -1.63998000 & -0.12665300 & & \\
\hline $\mathrm{C}$ & -1.57576500 & 1.93245900 & -0.88565300 & & \\
\hline $\mathrm{C}$ & 2.15447400 & 2.99275200 & -0.11312300 & & \\
\hline $\mathrm{C}$ & 1.19283200 & -2.76999600 & -0.78452400 & & \\
\hline $\mathrm{C}$ & -4.03823200 & -0.07040200 & 1.11516800 & & \\
\hline $\mathrm{C}$ & -3.73107700 & -1.36990900 & 0.71310100 & & \\
\hline $\mathrm{C}$ & -3.75190500 & 2.40605200 & 1.06622500 & & \\
\hline $\mathrm{C}$ & 3.80216400 & -1.65994100 & 0.44910300 & & \\
\hline $\mathrm{C}$ & -2.36534800 & -3.05036400 & -0.57720900 & & \\
\hline $\mathrm{H}$ & 2.98873000 & 3.25976200 & -0.77545700 & & \\
\hline $\mathrm{H}$ & 1.27889300 & 3.55178700 & -0.43398000 & & \\
\hline $\mathrm{H}$ & 2.01998800 & -3.21339400 & -1.33718500 & & \\
\hline $\mathrm{H}$ & 1.08909300 & -3.34221900 & 0.14032100 & & \\
\hline $\mathrm{H}$ & 0.28439800 & -2.87713700 & -1.36718800 & & \\
\hline $\mathrm{H}$ & -4.87848900 & 0.09331500 & 1.78371000 & & \\
\hline $\mathrm{H}$ & -2.90541900 & 3.02580700 & 1.36699700 & & \\
\hline $\mathrm{H}$ & -4.23965200 & 2.92588200 & 0.23996000 & & \\
\hline $\mathrm{H}$ & -4.44882300 & 2.34256300 & 1.90389400 & & \\
\hline $\mathrm{H}$ & -1.90931600 & -3.05960200 & -1.56600900 & & \\
\hline $\mathrm{H}$ & 4.64098200 & 0.38797100 & 0.79917700 & & \\
\hline $\mathrm{H}$ & 3.24509500 & 2.94608800 & 1.47699300 & & \\
\hline $\mathrm{H}$ & -5.19650500 & -2.12701200 & 1.67733500 & & \\
\hline $\mathrm{H}$ & 4.42312100 & -3.38210100 & 0.76665500 & & \\
\hline $\mathrm{H}$ & -3.29471700 & -3.61829800 & -0.61361200 & & \\
\hline $\mathrm{H}$ & -1.69389100 & -3.57191200 & 0.11201300 & & \\
\hline \multicolumn{6}{|c|}{ Radical } \\
\hline \multicolumn{4}{|c|}{ Cartesian Coordinates } & \multicolumn{2}{|l|}{ Frequency and Energy } \\
\hline 0 & -0.76474300 & -0.82376400 & -1.35735100 & \multirow{2}{*}{\multicolumn{2}{|c|}{$\begin{array}{l}\text { Zero-point correction }= \\
\text { Thermal correction to Energy= }\end{array}$}} \\
\hline $\mathrm{O}$ & -0.26351300 & 1.89168900 & -1.18834400 & Thermal correction to Energy= & \\
\hline 0 & 3.97646600 & 1.15862200 & 0.64515900 & \multicolumn{2}{|l|}{ Thermal correction to Enthalpy= } \\
\hline 0 & 2.33494500 & 3.37485400 & 1.29780600 & \multicolumn{2}{|c|}{ Thermal correction to Gibbs Free Energy= $\quad 0.252112$} \\
\hline $\mathrm{O}$ & -4.40543500 & -2.52260800 & 1.19077500 & Sum of electronic and zero-point Energies= & -1296.746222 \\
\hline $\mathrm{O}$ & -2.23426100 & 2.84894800 & -1.28767100 & Sum of electronic and thermal Energies= & -1296.723033 \\
\hline $\mathrm{O}$ & 3.59420100 & -2.96176600 & 0.44965300 & Sum of electronic and thermal Enthalpies= & -1296.722088 \\
\hline $\mathrm{O}$ & 4.87428900 & -1.19359000 & 0.84794100 & \multirow{5}{*}{\multicolumn{2}{|c|}{ Sum of electronic and thermal Free Energies= }} \\
\hline $\mathrm{C}$ & 0.45885700 & -0.38541800 & -0.84650600 & & \\
\hline $\mathrm{C}$ & 0.65978600 & 0.99482800 & -0.70834000 & & \\
\hline $\mathrm{C}$ & 1.46847000 & -1.29597900 & -0.56269800 & & \\
\hline $\mathrm{C}$ & 1.84525600 & 1.51766000 & -0.21669100 & & \\
\hline
\end{tabular}




\begin{tabular}{|c|c|c|c|c|c|}
\hline \multirow{2}{*}{\multicolumn{6}{|c|}{$\begin{array}{lll}-2.23241500 & 0.72620600 & -0.22618900\end{array}$}} \\
\hline & & & & & \\
\hline C & -1.86385200 & -0.58171500 & -0.54827400 & & \\
\hline $\mathrm{C}$ & 2.68695800 & -0.79146600 & -0.01871700 & & \\
\hline C & 2.85203000 & 0.61042600 & 0.14580600 & & \\
\hline C & -3.33978500 & 0.95505500 & 0.63213400 & & \\
\hline C & -2.61325400 & -1.72024600 & -0.12826300 & & \\
\hline C & -1.61958500 & 1.88697600 & -0.92365100 & & \\
\hline C & 2.09036100 & 3.00518200 & -0.05352800 & & \\
\hline C & 1.25069700 & -2.74675100 & -0.91979800 & & \\
\hline C & -4.02963700 & -0.15243600 & 1.12812800 & & \\
\hline $\mathrm{C}$ & -3.70812000 & -1.44483100 & 0.74927900 & & \\
\hline C & -3.79531500 & 2.33451900 & 1.03381400 & & \\
\hline $\mathrm{C}$ & 3.79889000 & -1.63334600 & 0.44431800 & & \\
\hline $\mathrm{C}$ & -2.33889500 & -3.02608600 & -0.54395900 & & \\
\hline $\mathrm{H}$ & 2.92999400 & 3.29782300 & -0.69785100 & & \\
\hline $\mathrm{H}$ & 1.21141500 & 3.55798900 & -0.37598300 & & \\
\hline $\mathrm{H}$ & 2.14399000 & -3.17783200 & -1.36599600 & & \\
\hline $\mathrm{H}$ & 1.00580900 & -3.34864500 & -0.04124100 & & \\
\hline $\mathrm{H}$ & 0.43405200 & -2.82341000 & -1.63149600 & & \\
\hline $\mathrm{H}$ & -4.86277600 & 0.00710000 & 1.80671300 & & \\
\hline $\mathrm{H}$ & -2.95896900 & 2.97440700 & 1.32253800 & & \\
\hline $\mathrm{H}$ & -4.29695800 & 2.83634400 & 0.20378600 & & \\
\hline $\mathrm{H}$ & -4.48686700 & 2.27086500 & 1.87582300 & & \\
\hline $\mathrm{H}$ & -1.54989700 & -3.23136100 & -1.24725900 & & \\
\hline $\mathrm{H}$ & 4.61084900 & 0.42217200 & 0.82537800 & & \\
\hline $\mathrm{H}$ & 3.15930800 & 2.94448900 & 1.55104000 & & \\
\hline $\mathrm{H}$ & -5.12125000 & -2.22800300 & 1.76413300 & & \\
\hline $\mathrm{H}$ & 4.41125500 & -3.35296600 & 0.79493800 & & \\
\hline $\mathrm{H}$ & -2.94822300 & -3.84351700 & -0.18946100 & & \\
\hline \multicolumn{6}{|c|}{ Anion } \\
\hline \multicolumn{4}{|c|}{ Cartesian Coordinates } & \multicolumn{2}{|l|}{ Frequency and Energy } \\
\hline $\mathrm{O}$ & -0.79556900 & -0.80080600 & -1.35253800 & Zero-point correction $=$ & (Hartree/Particle) \\
\hline $\mathrm{O}$ & -0.20444700 & 1.89696600 & -1.23035900 & Thermal correction to Energy= & 7374 \\
\hline 0 & 4.02323100 & 1.00005300 & 0.65855800 & Thermal correction to Enthalpy= & 28319 \\
\hline $\mathrm{O}$ & 2.72662900 & 3.30728100 & 1.14467800 & Thermal correction to Gibbs Free Energy= & 0.252274 \\
\hline $\mathrm{O}$ & -4.50441600 & -2.35911300 & 1.14488500 & Sum of electronic and zero-point Energies= & -1296.879604 \\
\hline $\mathrm{O}$ & -2.13197300 & 2.93970800 & -1.22623600 & Sum of electronic and thermal Energies= & -1296.856488 \\
\hline 0 & 3.61661500 & -3.06179300 & 0.40509100 & Sum of electronic and thermal Enthalpies= & -1296.855544 \\
\hline $\mathrm{O}$ & 4.85766600 & -1.29579500 & 0.91303900 & Sum of electronic and thermal Free Energies= & -1296.931588 \\
\hline C & 0.46381600 & -0.39970000 & -0.84193600 & & \\
\hline $\mathrm{C}$ & 0.71370000 & 0.97036900 & -0.72132700 & & \\
\hline $\mathrm{C}$ & 1.43500500 & -1.33899800 & -0.50577500 & & \\
\hline C & 1.90163700 & 1.46112900 & -0.22875900 & & \\
\hline $\mathrm{C}$ & -2.16579900 & 0.81073100 & -0.18761700 & & \\
\hline C & -1.86697100 & -0.52152900 & -0.53793000 & & \\
\hline C & 2.66850600 & -0.87882900 & 0.02264400 & & \\
\hline C & 2.91200500 & 0.53117000 & 0.17769800 & & \\
\hline C & -3.24553900 & 1.06874000 & 0.68449700 & & \\
\hline C & -2.67177200 & -1.59691200 & -0.13989400 & & \\
\hline C & -1.51724200 & 1.95331800 & -0.89657100 & & \\
\hline C & 2.22090000 & 2.93779600 & -0.13304900 & & \\
\hline C & 1.15361000 & -2.80143200 & -0.76373200 & & \\
\hline C & -3.99170100 & -0.00649000 & 1.15154100 & & \\
\hline C & -3.73256000 & -1.30779300 & 0.72150900 & & \\
\hline
\end{tabular}




\begin{tabular}{|c|c|c|c|c|c|}
\hline \multirow{2}{*}{\multicolumn{3}{|c|}{$\begin{array}{lll}-3.61996100 & 2.46061700 & 1.13694200\end{array}$}} & & & \\
\hline & -3.61996100 & 2.46061700 & 1.13694200 & & \\
\hline C & 3.73444100 & -1.84748100 & 0.45170000 & & \\
\hline $\mathrm{C}$ & -2.42610300 & -3.00798100 & -0.60573900 & & \\
\hline $\mathrm{H}$ & 2.95438900 & 3.18638600 & -0.91854700 & & \\
\hline $\mathrm{H}$ & 1.32772700 & 3.53439000 & -0.31833000 & & \\
\hline $\mathrm{H}$ & 1.99921100 & -3.26886000 & -1.26443500 & & \\
\hline $\mathrm{H}$ & 1.02955900 & -3.35311300 & 0.17191200 & & \\
\hline $\mathrm{H}$ & 0.25718600 & -2.91014600 & -1.36915200 & & \\
\hline $\mathrm{H}$ & -4.81078100 & 0.17210600 & 1.84379200 & & \\
\hline $\mathrm{H}$ & -2.74393300 & 3.03599900 & 1.44253900 & & \\
\hline $\mathrm{H}$ & -4.08993100 & 3.01942200 & 0.32629400 & & \\
\hline $\mathrm{H}$ & -4.31037100 & 2.40684800 & 1.98223200 & & \\
\hline $\mathrm{H}$ & -1.99181400 & -3.01192900 & -1.60448100 & & \\
\hline $\mathrm{H}$ & 4.72505800 & -0.28038600 & 0.88935300 & & \\
\hline $\mathrm{H}$ & 3.43931200 & 2.65768400 & 1.28955800 & & \\
\hline $\mathrm{H}$ & -5.18869000 & -2.01738900 & 1.72884900 & & \\
\hline $\mathrm{H}$ & -3.36166900 & -3.56816400 & -0.61838600 & & \\
\hline $\mathrm{H}$ & -1.73297900 & -3.53522500 & 0.05664500 & & \\
\hline \multicolumn{6}{|c|}{ Radical cation } \\
\hline \multicolumn{4}{|c|}{ Cartesian Coordinates } & \multicolumn{2}{|l|}{ Frequency and Energy } \\
\hline 0 & -0.72456900 & -1.02315000 & -0.25513100 & Zero-point correction $=$ & (Hartree/Particle) \\
\hline $\mathrm{O}$ & -0.30630200 & 1.66054500 & -0.85915200 & Thermal correction to Energy= & 0075 \\
\hline $\mathrm{O}$ & 4.28637700 & 1.01651300 & -0.25688100 & Thermal correction to Enthalpy= & 1019 \\
\hline $\mathrm{O}$ & 2.89494000 & 3.47100600 & 0.35031200 & Thermal correction to Gibbs Free Energy $=$ & 0.261503 \\
\hline $\mathrm{O}$ & -5.21702300 & -2.15362400 & -0.21513800 & Sum of electronic and zero-point Energies= & -1297.112420 \\
\hline $\mathrm{O}$ & -1.37923800 & 2.96165300 & 0.63522700 & Sum of electronic and thermal Energies= & -1297.088335 \\
\hline $\mathrm{O}$ & 3.90242300 & -2.98211400 & 0.63059100 & Sum of electronic and thermal Enthalpies= & -1297.087391 \\
\hline $\mathrm{O}$ & 5.20887300 & -1.20863700 & 0.33089500 & Sum of electronic and thermal Free Energies= & -1297.166907 \\
\hline $\mathrm{C}$ & 0.49463500 & -0.48171500 & -0.18718000 & & \\
\hline $\mathrm{C}$ & 0.73668400 & 0.88699100 & -0.48757800 & & \\
\hline $\mathrm{C}$ & 1.55860600 & -1.39716500 & 0.11073000 & & \\
\hline $\mathrm{C}$ & 2.01461300 & 1.39264400 & -0.50947500 & & \\
\hline $\mathrm{C}$ & -2.33908700 & 0.82220000 & 0.14487200 & & \\
\hline $\mathrm{C}$ & -2.00896300 & -0.51900600 & -0.17595500 & & \\
\hline $\mathrm{C}$ & 2.86165700 & -0.89462400 & 0.08462400 & & \\
\hline $\mathrm{C}$ & 3.08268500 & 0.49887400 & -0.22266500 & & \\
\hline $\mathrm{C}$ & -3.68784000 & 1.13239900 & 0.42824100 & & \\
\hline $\mathrm{C}$ & -2.96490700 & -1.53280500 & -0.31908700 & & \\
\hline $\mathrm{C}$ & -1.35606400 & 1.90398700 & 0.09717400 & & \\
\hline C & 2.31415600 & 2.85638000 & -0.78490500 & & \\
\hline $\mathrm{C}$ & 1.20602300 & -2.82161200 & 0.44275400 & & \\
\hline $\mathrm{C}$ & -4.64461700 & 0.12205900 & 0.30459800 & & \\
\hline $\mathrm{C}$ & -4.30517100 & -1.17279100 & -0.07867500 & & \\
\hline $\mathrm{C}$ & -4.14604200 & 2.50993100 & 0.82770000 & & \\
\hline $\mathrm{C}$ & 4.08491700 & -1.69514000 & 0.35832000 & & \\
\hline $\mathrm{C}$ & -2.62894900 & -2.95272900 & -0.67809300 & & \\
\hline $\mathrm{H}$ & 2.95219500 & 2.93739400 & -1.67170200 & & \\
\hline $\mathrm{H}$ & 1.37891200 & 3.37277900 & -0.98708100 & & \\
\hline $\mathrm{H}$ & 1.64451200 & -3.50641800 & -0.28565600 & & \\
\hline $\mathrm{H}$ & 1.61366500 & -3.10100100 & 1.41476400 & & \\
\hline $\mathrm{H}$ & 0.13153200 & -2.96424200 & 0.45812400 & & \\
\hline $\mathrm{H}$ & -5.68436900 & 0.35993300 & 0.50293900 & & \\
\hline $\mathrm{H}$ & -3.69844700 & 2.81356300 & 1.77589700 & & \\
\hline $\mathrm{H}$ & -3.85004000 & 3.26333000 & 0.09432600 & & \\
\hline
\end{tabular}




\begin{tabular}{|c|c|c|c|c|c|}
\hline $\mathrm{H}$ & -5.23080900 & 2.53084000 & 0.92784100 & & \\
\hline $\mathrm{H}$ & -1.69039800 & -3.02439500 & -1.22270100 & & \\
\hline $\mathrm{H}$ & 4.94794800 & 0.28901700 & -0.04075400 & & \\
\hline $\mathrm{H}$ & 3.84220500 & 3.30079700 & 0.34041400 & & \\
\hline $\mathrm{H}$ & -6.10686300 & -1.82650900 & -0.03634900 & & \\
\hline $\mathrm{H}$ & 4.77693100 & -3.37823200 & 0.78323000 & & \\
\hline $\mathrm{H}$ & -3.42463600 & -3.37523700 & -1.29155600 & & \\
\hline $\mathrm{H}$ & -2.56019100 & -3.57641500 & 0.22009600 & & \\
\hline \multicolumn{6}{|c|}{ Name of compound (11) } \\
\hline \multicolumn{4}{|c|}{ Cartesian Coordinates } & \multicolumn{2}{|l|}{ Frequency and Energy } \\
\hline $\mathrm{Cl}$ & 1.75749400 & -3.04064500 & -0.82815600 & Zero-point correction $=$ & (Hartree/Particle) \\
\hline $\mathrm{Cl}$ & 5.37038200 & 0.13446200 & 1.58384100 & Thermal correction to Energy= & 8412 \\
\hline 0 & 0.34634100 & -0.50528300 & -1.44788700 & Thermal correction to Enthalpy= & 19357 \\
\hline 0 & 0.02150000 & 2.28017000 & -1.25631700 & Thermal correction to Gibbs Free Energy= & 0.235195 \\
\hline 0 & -4.19410500 & 1.64524000 & 0.88485600 & Sum of electronic and zero-point Energies= & -2141.359712 \\
\hline 0 & 4.21359700 & -2.33432400 & 0.67756600 & Sum of electronic and thermal Energies= & -2141.333811 \\
\hline 0 & 2.04045100 & 3.06072300 & -1.60391000 & Sum of electronic and thermal Enthalpies= & -2141.332866 \\
\hline 0 & -5.28050000 & -0.79047600 & -0.01127500 & Sum of electronic and thermal Free Energies= & -2141.417028 \\
\hline 0 & -3.98069000 & -1.99714500 & 1.37325000 & & \\
\hline $\mathrm{C}$ & -0.80085700 & 0.03530400 & -0.85568100 & & \\
\hline $\mathrm{C}$ & -1.84665500 & -0.80480000 & -0.45616300 & & \\
\hline $\mathrm{C}$ & 1.98953400 & 0.98188700 & -0.46428200 & & \\
\hline $\mathrm{C}$ & -0.90988600 & 1.41211900 & -0.72780700 & & \\
\hline $\mathrm{C}$ & 1.51508100 & -0.30620300 & -0.75506400 & & \\
\hline $\mathrm{C}$ & -2.99356600 & -0.21972800 & 0.10088600 & & \\
\hline $\mathrm{C}$ & 3.18236900 & 1.14992000 & 0.28131700 & & \\
\hline $\mathrm{C}$ & -3.07807400 & 1.17581800 & 0.27361100 & & \\
\hline C & -2.03432000 & 1.99276300 & -0.15459400 & & \\
\hline $\mathrm{C}$ & 1.38597800 & 2.17037000 & -1.13955600 & & \\
\hline $\mathrm{C}$ & 2.26565900 & -1.42136600 & -0.39362000 & & \\
\hline $\mathrm{C}$ & -1.74263800 & -2.29509000 & -0.66647700 & & \\
\hline $\mathrm{C}$ & 3.88427000 & 0.00805600 & 0.66652500 & & \\
\hline C & 3.46537200 & -1.28258900 & 0.31284100 & & \\
\hline C & 3.70112300 & 2.50703900 & 0.68567800 & & \\
\hline $\mathrm{C}$ & -4.11215300 & -1.09891100 & 0.57795000 & & \\
\hline $\mathrm{C}$ & -4.33776500 & 3.04937300 & 1.06556200 & & \\
\hline $\mathrm{C}$ & -6.42071700 & -1.54589400 & 0.43687000 & & \\
\hline $\mathrm{H}$ & -2.06447900 & 3.06918000 & -0.07479600 & & \\
\hline $\mathrm{H}$ & -1.40468800 & -2.79239100 & 0.24628300 & & \\
\hline $\mathrm{H}$ & -2.71153400 & -2.72319100 & -0.92355600 & & \\
\hline $\mathrm{H}$ & -1.03660600 & -2.52050100 & -1.46187300 & & \\
\hline $\mathrm{H}$ & 4.02933200 & 2.48417400 & 1.72582000 & & \\
\hline $\mathrm{H}$ & 4.56621800 & 2.78286300 & 0.07614800 & & \\
\hline $\mathrm{H}$ & 2.95485400 & 3.28527800 & 0.56210900 & & \\
\hline $\mathrm{H}$ & -4.34589100 & 3.57582600 & 0.10566700 & & \\
\hline $\mathrm{H}$ & -3.54251600 & 3.45383000 & 1.70069200 & & \\
\hline $\mathrm{H}$ & -5.29794200 & 3.18445800 & 1.55942100 & & \\
\hline $\mathrm{H}$ & 3.78934200 & -3.14540800 & 0.36253700 & & \\
\hline $\mathrm{H}$ & -6.27877100 & -2.60954400 & 0.23984000 & & \\
\hline $\mathrm{H}$ & -7.26442600 & -1.15930900 & -0.13007000 & & \\
\hline $\mathrm{H}$ & -6.57605800 & -1.39980100 & 1.50688900 & & \\
\hline \multicolumn{6}{|c|}{ Radical } \\
\hline \multicolumn{4}{|c|}{ Cartesian Coordinates } & \multicolumn{2}{|l|}{ Frequency and Energy } \\
\hline $\mathrm{Cl}$ & 1.78801700 & -3.09167300 & -0.83863600 & Zero-point correction= & (Hartree/Particle) \\
\hline
\end{tabular}




\begin{tabular}{|c|c|c|c|c|c|}
\hline $\mathrm{Cl}$ & 5.32154100 & 0.17195600 & 1.67084300 & \multicolumn{2}{|l|}{ Thermal correction to Energy= } \\
\hline 0 & 0.36041600 & -0.55070900 & -1.46962800 & \multicolumn{2}{|l|}{ Thermal correction to Enthalpy= } \\
\hline 0 & 0.07467900 & 2.23986900 & -1.30581900 & Thermal correction to Gibbs Free Energy= & 0.220632 \\
\hline $\mathrm{O}$ & -4.13978200 & 1.68221700 & 0.86650200 & Sum of electronic and zero-point Energies= & -2140.724863 \\
\hline 0 & 4.18874200 & -2.33325000 & 0.68581800 & Sum of electronic and thermal Energies= & -2140.699038 \\
\hline 0 & 2.12572600 & 2.88153500 & -1.76507900 & Sum of electronic and thermal Enthalpies= & -2140.698094 \\
\hline 0 & -5.24198100 & -0.78594400 & -0.00935300 & Sum of electronic and thermal Free Energies= & -2140.783307 \\
\hline 0 & -3.96381900 & -1.91069200 & 1.46311000 & & \\
\hline $\mathrm{C}$ & -0.77550900 & 0.00942900 & -0.87139900 & & \\
\hline $\mathrm{C}$ & -1.82678000 & -0.81232500 & -0.44671600 & & \\
\hline C & 2.00496200 & 0.91067800 & -0.47443600 & & \\
\hline $\mathrm{C}$ & -0.86979500 & 1.38906000 & -0.75952700 & & \\
\hline $\mathrm{C}$ & 1.52729500 & -0.39371200 & -0.76133200 & & \\
\hline $\mathrm{C}$ & -2.95975500 & -0.20361100 & 0.11059700 & & \\
\hline $\mathrm{C}$ & 3.17174400 & 1.12850300 & 0.31614900 & & \\
\hline $\mathrm{C}$ & -3.03034600 & 1.19468600 & 0.25968300 & & \\
\hline $\mathrm{C}$ & -1.98122400 & 1.99449500 & -0.18871300 & & \\
\hline C & 1.42483400 & 2.07400700 & -1.22311200 & & \\
\hline $\mathrm{C}$ & 2.26729100 & -1.49766700 & -0.40380300 & & \\
\hline $\mathrm{C}$ & -1.74583100 & -2.30782500 & -0.62511900 & & \\
\hline $\mathrm{C}$ & 3.88194800 & 0.01687700 & 0.71975100 & & \\
\hline $\mathrm{C}$ & 3.51495200 & -1.36031600 & 0.35750700 & & \\
\hline $\mathrm{C}$ & 3.62107000 & 2.51429600 & 0.70877400 & & \\
\hline $\mathrm{C}$ & -4.08527100 & -1.06001400 & 0.61683700 & & \\
\hline $\mathrm{C}$ & -4.27333100 & 3.09045800 & 1.02603000 & & \\
\hline C & -6.38988600 & -1.52119800 & 0.45563800 & & \\
\hline $\mathrm{H}$ & -1.99972400 & 3.07234800 & -0.12637500 & & \\
\hline $\mathrm{H}$ & -1.45740600 & -2.79292200 & 0.31076000 & & \\
\hline $\mathrm{H}$ & -2.71439200 & -2.71781900 & -0.91375300 & & \\
\hline $\mathrm{H}$ & -1.01509800 & -2.56605400 & -1.38692800 & & \\
\hline $\mathrm{H}$ & 3.93901500 & 2.51977100 & 1.75193400 & & \\
\hline $\mathrm{H}$ & 4.47424000 & 2.82762500 & 0.10142900 & & \\
\hline $\mathrm{H}$ & 2.83556200 & 3.25323100 & 0.57554100 & & \\
\hline $\mathrm{H}$ & -4.28106500 & 3.60151400 & 0.05792300 & & \\
\hline $\mathrm{H}$ & -3.47333800 & 3.49838600 & 1.65278900 & & \\
\hline $\mathrm{H}$ & -5.23085300 & 3.23964100 & 1.52068600 & & \\
\hline $\mathrm{H}$ & -6.23708000 & -2.59346000 & 0.32582200 & & \\
\hline $\mathrm{H}$ & -7.22114200 & -1.17419200 & -0.15352300 & & \\
\hline $\mathrm{H}$ & -6.57127700 & -1.31201000 & 1.51090500 & & \\
\hline \multicolumn{6}{|c|}{ Anion } \\
\hline \multicolumn{4}{|c|}{ Cartesian Coordinates } & \multicolumn{2}{|l|}{ Frequency and Energy } \\
\hline $\mathrm{Cl}$ & 1.75010000 & -3.07509800 & -0.87410700 & $\begin{array}{l}\text { Zero-point correction }= \\
\text { Thermal correction to Energy }\end{array}$ & 0.278932 (Hartree/Particle) \\
\hline $\mathrm{Cl}$ & 5.48013000 & 0.08741300 & 1.48946200 & \multicolumn{2}{|l|}{ Thermal correction to Energy= } \\
\hline $\mathrm{O}$ & 0.28565300 & -0.59194500 & -1.29949000 & \multicolumn{2}{|l|}{ Thermal correction to Enthalpy= } \\
\hline 0 & 0.04105700 & 2.16472000 & -1.40279500 & Thermal correction to Gibbs Free Energy= & 0.221414 \\
\hline $\mathrm{O}$ & -4.29551500 & 1.87171000 & 0.59066200 & Sum of electronic and zero-point Energies= & -2140.849333 \\
\hline $\mathrm{O}$ & 4.30051500 & -2.38646400 & 0.44000800 & Sum of electronic and thermal Energies= & -2140.823577 \\
\hline $\mathrm{O}$ & 1.99726300 & 3.16159000 & -1.28577200 & Sum of electronic and thermal Enthalpies= & -2140.822632 \\
\hline $\mathrm{O}$ & -4.74825600 & -1.68489400 & -0.15642800 & Sum of electronic and thermal Free Energies= & -2140.906851 \\
\hline $\mathrm{O}$ & -4.61483800 & -0.76045200 & 1.88885800 & & \\
\hline $\mathrm{C}$ & -0.83275300 & 0.01842400 & -0.76727000 & & \\
\hline $\mathrm{C}$ & -1.89654000 & -0.74905100 & -0.27475000 & & \\
\hline $\mathrm{C}$ & 1.96061500 & 0.95902100 & -0.37960800 & & \\
\hline $\mathrm{C}$ & -0.92624500 & 1.40419000 & -0.81652000 & & \\
\hline
\end{tabular}




\begin{tabular}{|c|c|c|c|c|c|}
\hline \multirow{2}{*}{\multicolumn{6}{|c|}{$\begin{array}{lll}1.50652700 & -0.36123100 & -0.67363300\end{array}$}} \\
\hline & & & & & \\
\hline C & -3.04690300 & -0.08412000 & 0.18524800 & & \\
\hline C & 3.20969800 & 1.09114900 & 0.32889300 & & \\
\hline C & -3.12634200 & 1.31834900 & 0.14846300 & & \\
\hline C & -2.06530600 & 2.06179600 & -0.35736600 & & \\
\hline C & 1.40798800 & 2.14216600 & -1.01524000 & & \\
\hline C & 2.28861900 & -1.46202500 & -0.41684700 & & \\
\hline C & -1.79730900 & -2.25403100 & -0.25037300 & & \\
\hline $\mathrm{C}$ & 3.94435100 & -0.03497500 & 0.60706100 & & \\
\hline $\mathrm{C}$ & 3.59504100 & -1.39926200 & 0.22269700 & & \\
\hline $\mathrm{C}$ & 3.68715300 & 2.44339000 & 0.81251100 & & \\
\hline $\mathrm{C}$ & -4.19872900 & -0.84650300 & 0.76078400 & & \\
\hline $\mathrm{C}$ & -4.39673200 & 3.28401100 & 0.63294000 & & \\
\hline $\mathrm{C}$ & -5.82424200 & -2.50016200 & 0.32768300 & & \\
\hline $\mathrm{H}$ & -2.08793200 & 3.13907100 & -0.43314900 & & \\
\hline $\mathrm{H}$ & -2.16031700 & -2.65518900 & 0.69953100 & & \\
\hline $\mathrm{H}$ & -2.40824600 & -2.69067600 & -1.04512400 & & \\
\hline $\mathrm{H}$ & -0.76952500 & -2.57464100 & -0.39448400 & & \\
\hline $\mathrm{H}$ & 4.17535200 & 2.33797900 & 1.78158000 & & \\
\hline $\mathrm{H}$ & 4.41453100 & 2.87333800 & 0.11797700 & & \\
\hline $\mathrm{H}$ & 2.86819800 & 3.15328200 & 0.89479300 & & \\
\hline $\mathrm{H}$ & -4.33172900 & 3.72656400 & -0.36819100 & & \\
\hline $\mathrm{H}$ & -3.62224100 & 3.72413500 & 1.27113400 & & \\
\hline $\mathrm{H}$ & -5.37711100 & 3.49799600 & 1.05722100 & & \\
\hline $\mathrm{H}$ & -5.48856600 & -3.13669200 & 1.14909500 & & \\
\hline $\mathrm{H}$ & -6.13686200 & -3.10619300 & -0.52083100 & & \\
\hline $\mathrm{H}$ & -6.64984500 & -1.87927500 & 0.68102600 & & \\
\hline \multicolumn{6}{|c|}{ Radical cation } \\
\hline \multicolumn{4}{|c|}{ Cartesian Coordinates } & \multicolumn{2}{|l|}{ Frequency and Energy } \\
\hline $\mathrm{Cl}$ & 1.93640900 & -2.99936200 & 0.53411100 & Zero-point correction $=$ & (Hartree/Particle) \\
\hline $\mathrm{Cl}$ & 6.06005800 & 0.33764600 & -0.26326300 & Thermal correction to Energy= & 7659 \\
\hline $\mathrm{O}$ & 0.30572200 & -0.71163800 & 0.09996300 & Thermal correction to Enthalpy $=$ & 18603 \\
\hline $\mathrm{O}$ & 0.10341600 & 2.06000300 & 0.69412900 & Thermal correction to Gibbs Free Energy $=$ & 0.232871 \\
\hline 0 & -4.61530800 & 1.51601400 & 0.57920400 & Sum of electronic and zero-point Energies= & -2141.084338 \\
\hline $\mathrm{O}$ & 4.77819600 & -2.14559700 & 0.29576200 & Sum of electronic and thermal Energies= & -2141.058008 \\
\hline $\mathrm{O}$ & 1.16309600 & 3.02581400 & -1.04207200 & Sum of electronic and thermal Enthalpies= & -2141.057063 \\
\hline 0 & -5.21457700 & -0.68103700 & -1.17245900 & Sum of electronic and thermal Free Energies= & -2141.142795 \\
\hline 0 & -4.82181300 & -2.02318200 & 0.60128000 & & \\
\hline $\mathrm{C}$ & -0.86818900 & -0.06697800 & 0.14715100 & & \\
\hline $\mathrm{C}$ & -2.01430500 & -0.89191100 & -0.05177900 & & \\
\hline $\mathrm{C}$ & 2.09325400 & 0.98926400 & -0.16610400 & & \\
\hline $\mathrm{C}$ & -0.99393300 & 1.31543800 & 0.46087300 & & \\
\hline $\mathrm{C}$ & 1.61365600 & -0.31396600 & 0.09505700 & & \\
\hline $\mathrm{C}$ & -3.25631100 & -0.30131400 & 0.06085900 & & \\
\hline $\mathrm{C}$ & 3.48107700 & 1.21701300 & -0.29947300 & & \\
\hline $\mathrm{C}$ & -3.37856400 & 1.08201100 & 0.42917500 & & \\
\hline$C$ & -2.23968100 & 1.88057100 & 0.61663300 & & \\
\hline C & 1.13950500 & 2.10133900 & -0.30218400 & & \\
\hline $\mathrm{C}$ & 2.50490900 & -1.38195200 & 0.24449100 & & \\
\hline $\mathrm{C}$ & -1.81024600 & -2.34480400 & -0.38328900 & & \\
\hline $\mathrm{C}$ & 4.35012700 & 0.12825700 & -0.12847900 & & \\
\hline C & 3.88432300 & -1.17196600 & 0.14997000 & & \\
\hline $\mathrm{C}$ & 4.06858900 & 2.57548700 & -0.57413000 & & \\
\hline $\mathrm{C}$ & -4.52271100 & -1.10905500 & -0.12070200 & & \\
\hline
\end{tabular}




\begin{tabular}{|c|c|c|c|c|c|}
\hline \multirow{2}{*}{\multicolumn{6}{|c|}{$\begin{array}{lll}-4.88740900 & 2.88113000 & 0.96670700\end{array}$}} \\
\hline & & & & & \\
\hline C & -6.46966500 & -1.36439100 & -1.43291300 & & \\
\hline $\mathrm{H}$ & -2.29781700 & 2.92448800 & 0.88704900 & & \\
\hline $\mathrm{H}$ & -1.22515400 & -2.82972500 & 0.40094100 & & \\
\hline $\mathrm{H}$ & -2.75628200 & -2.87066900 & -0.46937800 & & \\
\hline $\mathrm{H}$ & -1.25096200 & -2.45201800 & -1.31615400 & & \\
\hline $\mathrm{H}$ & 4.84971500 & 2.79814700 & 0.15519400 & & \\
\hline $\mathrm{H}$ & 4.54385200 & 2.58020000 & -1.55948700 & & \\
\hline $\mathrm{H}$ & 3.32977000 & 3.36820000 & -0.55939100 & & \\
\hline $\mathrm{H}$ & -4.51237800 & 3.56906300 & 0.20695300 & & \\
\hline $\mathrm{H}$ & -4.44294400 & 3.09209900 & 1.94097400 & & \\
\hline $\mathrm{H}$ & -5.96964600 & 2.94471400 & 1.02719700 & & \\
\hline $\mathrm{H}$ & 4.33795000 & -2.98956400 & 0.47597200 & & \\
\hline $\mathrm{H}$ & -6.28801000 & -2.42373300 & -1.61179500 & & \\
\hline $\mathrm{H}$ & -6.87511100 & -0.88408000 & -2.31858400 & & \\
\hline $\mathrm{H}$ & -7.13934400 & -1.24598800 & -0.58152700 & & \\
\hline \multicolumn{6}{|c|}{ Name of compound (12) } \\
\hline \multicolumn{4}{|c|}{ Cartesian Coordinates } & \multicolumn{2}{|l|}{ Frequency and Energy } \\
\hline $\mathrm{O}$ & -1.14442700 & -0.47567700 & -1.76632000 & Zero-point correction $=$ & 3 (Hartree/Particle) \\
\hline $\mathrm{O}$ & 1.14096400 & 1.12788400 & -1.63016400 & Thermal correction to Energy= & 5409 \\
\hline $\mathrm{O}$ & -1.13075200 & 4.53275500 & 0.55797400 & Thermal correction to Enthalpy= & 56353 \\
\hline $\mathrm{O}$ & 2.79211900 & -0.03506400 & 1.18194200 & Thermal correction to Gibbs Free Energy $=$ & 0.453184 \\
\hline $\mathrm{O}$ & -0.86763100 & -4.76153800 & 0.32002300 & Sum of electronic and zero-point Energies= & -1610.561113 \\
\hline $\mathrm{O}$ & 2.81332900 & -0.22491300 & -2.04364800 & Sum of electronic and thermal Energies= & -1610.527693 \\
\hline $\mathrm{O}$ & -3.67052200 & 4.42323900 & 0.78914900 & Sum of electronic and thermal Enthalpies= & -1610.526748 \\
\hline $\mathrm{O}$ & -4.78968900 & 2.51407300 & 0.55920000 & Sum of electronic and thermal Free Energies= & -1610.629917 \\
\hline $\mathrm{C}$ & -3.64240400 & 0.36690900 & -0.82371100 & & \\
\hline C & -3.96753500 & -0.47146100 & 0.42925800 & & \\
\hline C & -2.42070500 & 1.24834600 & -0.65746600 & & \\
\hline C & -5.18789100 & -1.37487800 & 0.22365600 & & \\
\hline C & -1.20526200 & 0.76206500 & -1.12844300 & & \\
\hline C & -2.45470700 & 2.55272800 & -0.07095200 & & \\
\hline C & 1.71417400 & -2.26259500 & -0.24467500 & & \\
\hline C & 6.08250000 & -0.84088600 & 0.56458300 & & \\
\hline C & 0.99204700 & -1.24618200 & -0.91921900 & & \\
\hline C & 3.18386100 & -2.13508400 & 0.06772300 & & \\
\hline C & -5.51775000 & -2.24073400 & 1.44478600 & & \\
\hline C & 4.94133100 & -0.99405600 & 1.59739000 & & \\
\hline C & -0.03486400 & 1.52110900 & -1.04224400 & & \\
\hline C & -0.38024100 & -1.43224800 & -1.12273800 & & \\
\hline C & 6.03328000 & 0.44875300 & -0.27380300 & & \\
\hline C & 3.55153000 & -0.95554800 & 0.98258300 & & \\
\hline C & -1.24653900 & 3.30303300 & 0.02041700 & & \\
\hline C & -0.05145300 & 2.77123300 & -0.46414900 & & \\
\hline C & 6.05131100 & 1.76403400 & 0.52234800 & & \\
\hline C & 1.05176200 & -3.40761300 & 0.17556700 & & \\
\hline C & 1.72069000 & -0.11837300 & -1.55480200 & & \\
\hline C & -1.03058300 & -2.59855700 & -0.73999600 & & \\
\hline C & -6.74503300 & -3.13387900 & 1.24109900 & & \\
\hline C & -0.30697900 & -3.59472800 & -0.09231700 & & \\
\hline C & -3.73587600 & 3.08987000 & 0.43173400 & & \\
\hline C & 7.30128400 & 1.97005100 & 1.38471200 & & \\
\hline $\mathrm{H}$ & -4.50998700 & 0.97599300 & -1.07083700 & & \\
\hline
\end{tabular}




\begin{tabular}{|c|c|c|c|c|c|}
\hline \multirow{2}{*}{\multicolumn{6}{|c|}{$-3.45733500-0.30516100-1.66295000$}} \\
\hline & & & & & \\
\hline $\mathrm{H}$ & -4.15382600 & 0.19981400 & 1.27069900 & & \\
\hline $\mathrm{H}$ & -3.09652600 & -1.08036500 & 0.69632400 & & \\
\hline $\mathrm{H}$ & -6.05686400 & -0.75246900 & -0.02274500 & & \\
\hline $\mathrm{H}$ & -5.02424000 & -2.02419600 & -0.64770900 & & \\
\hline $\mathrm{H}$ & 6.07829800 & -1.70132500 & -0.11289000 & & \\
\hline $\mathrm{H}$ & 7.02938200 & -0.89863900 & 1.11077000 & & \\
\hline $\mathrm{H}$ & 3.54651200 & -3.05885100 & 0.52566400 & & \\
\hline $\mathrm{H}$ & 3.75363500 & -1.99319700 & -0.85500800 & & \\
\hline $\mathrm{H}$ & 5.06890000 & -1.94751600 & 2.12285300 & & \\
\hline $\mathrm{H}$ & 4.98253000 & -0.19524500 & 2.33968200 & & \\
\hline $\mathrm{H}$ & -5.67950400 & -1.59034600 & 2.31225800 & & \\
\hline $\mathrm{H}$ & -4.64878500 & -2.86308000 & 1.69381600 & & \\
\hline $\mathrm{H}$ & 5.14254400 & 0.43724600 & -0.91049100 & & \\
\hline $\mathrm{H}$ & 6.89160600 & 0.43535000 & -0.95668800 & & \\
\hline $\mathrm{H}$ & 0.85619900 & 3.35486900 & -0.40218200 & & \\
\hline $\mathrm{H}$ & 5.97294100 & 2.58919600 & -0.19359200 & & \\
\hline $\mathrm{H}$ & 5.15393100 & 1.82948200 & 1.14616700 & & \\
\hline $\mathrm{H}$ & 1.58190800 & -4.18957500 & 0.70502500 & & \\
\hline $\mathrm{H}$ & -2.08779500 & -2.70510200 & -0.95159500 & & \\
\hline $\mathrm{H}$ & -6.60439600 & -3.81620700 & 0.39648200 & & \\
\hline $\mathrm{H}$ & -6.95027500 & -3.74051600 & 2.12736500 & & \\
\hline $\mathrm{H}$ & -7.63777100 & -2.53575100 & 1.03436900 & & \\
\hline $\mathrm{H}$ & 7.38424500 & 1.21980700 & 2.17676900 & & \\
\hline $\mathrm{H}$ & 8.21265900 & 1.91258500 & 0.78027300 & & \\
\hline $\mathrm{H}$ & 7.28664700 & 2.95055000 & 1.86839200 & & \\
\hline $\mathrm{H}$ & -2.00749400 & 4.85817200 & 0.81599700 & & \\
\hline $\mathrm{H}$ & -1.80156300 & -4.77420600 & 0.08461100 & & \\
\hline $\mathrm{H}$ & -4.54840400 & 4.65476400 & 1.12586700 & & \\
\hline \multicolumn{6}{|c|}{ 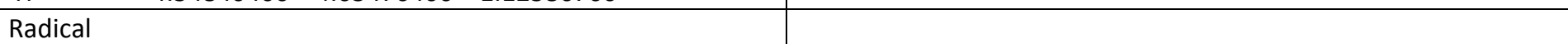 } \\
\hline \multicolumn{4}{|c|}{ Cartesian Coordinates } & \multicolumn{2}{|l|}{ Frequency and Energy } \\
\hline 0 & 1.19528300 & -0.45350300 & 1.60864700 & Zero-point correction $=$ & (Hartree/Particle) \\
\hline 0 & -1.11219600 & 1.14400500 & 1.58921500 & Thermal correction to Energy= & 1939 \\
\hline $\mathrm{O}$ & 1.26627200 & 4.48454100 & -0.61398200 & Thermal correction to Enthalpy $=\quad 0.542$ & 2883 \\
\hline $\mathrm{O}$ & -2.85211500 & 0.00443300 & -1.13220500 & Thermal correction to Gibbs Free Energy $=$ & 0.439607 \\
\hline $\mathrm{O}$ & 0.80760000 & -4.74215300 & -0.42567600 & Sum of electronic and zero-point Energies= & -1609.928953 \\
\hline $\mathrm{O}$ & -2.78487500 & -0.19364700 & 2.05407700 & Sum of electronic and thermal Energies= & -1609.895786 \\
\hline $\mathrm{O}$ & 3.78069900 & 4.40619100 & -0.94154000 & Sum of electronic and thermal Enthalpies= & -1609.894842 \\
\hline $\mathrm{O}$ & 4.89218600 & 2.56296500 & -0.44500000 & Sum of electronic and thermal Free Energies= & -1609.998118 \\
\hline $\mathrm{C}$ & 3.70431300 & 0.41453100 & 0.85423100 & & \\
\hline $\mathrm{C}$ & 4.06921700 & -0.43123300 & -0.38367200 & & \\
\hline $\mathrm{C}$ & 2.49949000 & 1.30396800 & 0.62879300 & & \\
\hline $\mathrm{C}$ & 5.25314200 & -1.36635800 & -0.11677800 & & \\
\hline $\mathrm{C}$ & 1.23599900 & 0.79031600 & 1.04583600 & & \\
\hline $\mathrm{C}$ & 2.53898600 & 2.56504700 & 0.03593100 & & \\
\hline $\mathrm{C}$ & -1.75075600 & -2.24454100 & 0.22808700 & & \\
\hline $\mathrm{C}$ & -6.13386300 & -0.83413900 & -0.51195100 & & \\
\hline $\mathrm{C}$ & -1.00524900 & -1.22326900 & 0.87218200 & & \\
\hline $\mathrm{C}$ & -3.22764500 & -2.10967300 & -0.04127400 & & \\
\hline $\mathrm{C}$ & 5.64178300 & -2.21523500 & -1.33254700 & & \\
\hline $\mathrm{C}$ & -4.99743300 & -0.96251500 & -1.55384700 & & \\
\hline $\mathrm{C}$ & 0.03960300 & 1.53907900 & 0.95837600 & & \\
\hline $\mathrm{C}$ & 0.37105300 & -1.41115300 & 1.01603700 & & \\
\hline $\mathrm{C}$ & -6.09166900 & 0.44531100 & 0.34192700 & & \\
\hline
\end{tabular}




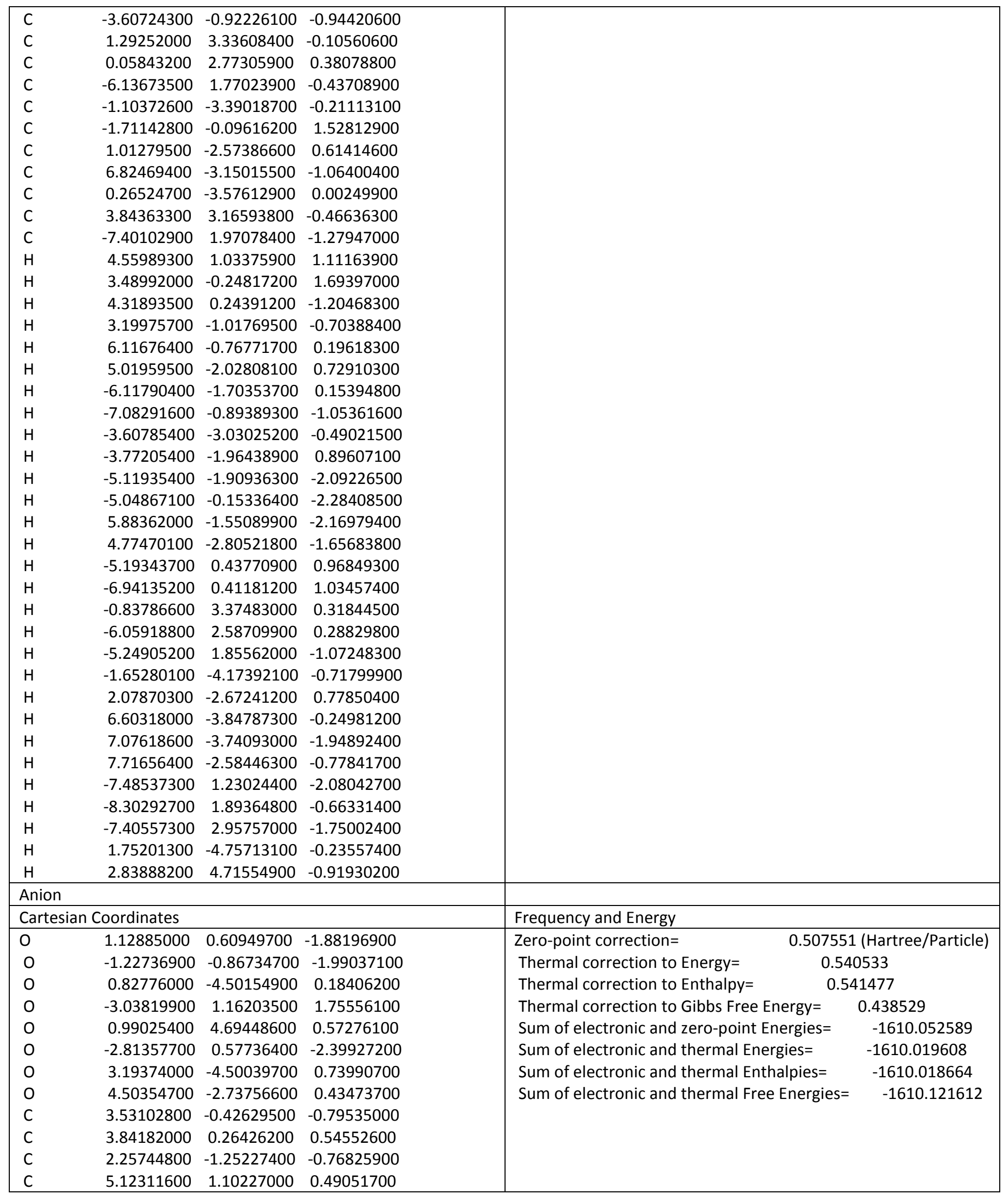




\begin{tabular}{|c|c|c|c|c|}
\hline & & & & \\
\hline$C$ & 1.10374500 & -0.68304900 & -1.30839600 & \\
\hline C & 2.18933700 & -2.56664600 & -0.24328600 & \\
\hline $\mathrm{C}$ & -1.71294000 & 2.46754600 & -0.43519200 & \\
\hline C & -5.36372800 & -0.19341500 & -0.08097000 & \\
\hline C & -1.00891800 & 1.43967500 & -1.11435000 & \\
\hline C & -3.21439400 & 2.44993000 & -0.27516500 & \\
\hline C & 5.44827400 & 1.81575800 & 1.80792100 & \\
\hline C & -5.19500900 & 1.11899400 & 0.72580500 & \\
\hline C & -0.10054000 & -1.38484000 & -1.33655100 & \\
\hline $\mathrm{C}$ & 0.38957800 & 1.54214700 & -1.20491500 & \\
\hline $\mathrm{C}$ & -4.69730800 & -1.43484200 & 0.53534000 & \\
\hline $\mathrm{C}$ & -3.73554100 & 1.53192700 & 0.83908500 & \\
\hline $\mathrm{C}$ & 0.93769100 & -3.30454800 & -0.26652700 & \\
\hline $\mathrm{C}$ & -0.20093400 & -2.65494800 & -0.83094400 & \\
\hline $\mathrm{C}$ & -5.14779200 & -1.81232600 & 1.95533000 & \\
\hline $\mathrm{C}$ & -1.01203300 & 3.52342200 & 0.13279500 & \\
\hline $\mathrm{C}$ & -1.74320500 & 0.37618700 & -1.86521700 & \\
\hline $\mathrm{C}$ & 1.07746100 & 2.62648900 & -0.66860500 & \\
\hline $\mathrm{C}$ & 6.73785800 & 2.64117200 & 1.75351700 & \\
\hline C & 0.37486600 & 3.61486600 & 0.00742000 & \\
\hline C & 3.39738500 & -3.25418800 & 0.33081300 & \\
\hline C & -6.64368200 & -2.11867500 & 2.08969800 & \\
\hline $\mathrm{H}$ & 4.36899300 & -1.07725500 & -1.03820400 & \\
\hline $\mathrm{H}$ & 3.43624000 & 0.33128600 & -1.57764900 & \\
\hline $\mathrm{H}$ & 3.95115700 & -0.50933600 & 1.30814200 & \\
\hline $\mathrm{H}$ & 2.99681100 & 0.89822700 & 0.84177300 & \\
\hline $\mathrm{H}$ & 5.96078200 & 0.44796900 & 0.21937500 & \\
\hline $\mathrm{H}$ & 5.04693100 & 1.84895500 & -0.31411500 & \\
\hline $\mathrm{H}$ & -4.95666900 & -0.04972200 & -1.08627400 & \\
\hline $\mathrm{H}$ & -6.43836000 & -0.36726900 & -0.20458300 & \\
\hline $\mathrm{H}$ & -3.56336300 & 3.45551300 & -0.00757400 & \\
\hline $\mathrm{H}$ & -3.69921800 & 2.18353300 & -1.21259000 & \\
\hline $\mathrm{H}$ & -5.76561100 & 1.91065600 & 0.22823300 & \\
\hline $\mathrm{H}$ & -5.58708600 & 0.99893000 & 1.73863600 & \\
\hline $\mathrm{H}$ & 5.52568500 & 1.06967000 & 2.60702000 & \\
\hline $\mathrm{H}$ & 4.60922000 & 2.46725000 & 2.08532400 & \\
\hline $\mathrm{H}$ & -3.61229100 & -1.29884000 & 0.54338600 & \\
\hline $\mathrm{H}$ & -4.88727500 & -2.28031600 & -0.13709800 & \\
\hline $\mathrm{H}$ & -1.13684800 & -3.19604900 & -0.87837400 & \\
\hline $\mathrm{H}$ & -4.57424600 & -2.69190800 & 2.26645300 & \\
\hline $\mathrm{H}$ & -4.86499000 & -1.01542900 & 2.65039500 & \\
\hline $\mathrm{H}$ & -1.53418800 & 4.30023400 & 0.67767200 & \\
\hline $\mathrm{H}$ & 2.15489400 & 2.66314800 & -0.78069000 & \\
\hline $\mathrm{H}$ & 6.68042100 & 3.41612600 & 0.98125900 & \\
\hline $\mathrm{H}$ & 6.94136500 & 3.13738900 & 2.70775900 & \\
\hline $\mathrm{H}$ & 7.59915500 & 2.00802800 & 1.51776100 & \\
\hline $\mathrm{H}$ & -7.26323800 & -1.24375500 & 1.86965500 & \\
\hline $\mathrm{H}$ & -6.94664700 & -2.91634500 & 1.40274700 & \\
\hline $\mathrm{H}$ & -6.88956800 & -2.44495700 & 3.10474200 & \\
\hline $\mathrm{H}$ & 2.18387400 & -4.70903700 & 0.57821600 & \\
\hline $\mathrm{H}$ & 1.94252700 & 4.59952600 & 0.46502300 & \\
\hline & tion & & & \\
\hline & Coordinates & & & Frequency and Energy \\
\hline 0 & 1.24892200 & -0.58920200 & 1.20218500 & 0.520950 (Hartree/Particle) \\
\hline
\end{tabular}




\begin{tabular}{|c|c|c|c|c|c|}
\hline 0 & -1.02171700 & 1.25228800 & 1.34377300 & \multicolumn{2}{|l|}{ Thermal correction to Energy= } \\
\hline 0 & 1.60946700 & 4.58164000 & -0.40344500 & Thermal correction to Enthalpy= & 5506 \\
\hline 0 & -2.88032800 & 0.04042600 & -1.05382200 & Thermal correction to Gibbs Free Energy= & 0.451046 \\
\hline 0 & 0.24955300 & -5.03474500 & -0.00974800 & Sum of electronic and zero-point Energies= & -1610.287785 \\
\hline 0 & -2.71897300 & 0.06660000 & 2.05608600 & Sum of electronic and thermal Energies= & -1610.254172 \\
\hline $\mathrm{O}$ & 4.13355900 & 4.37715700 & -0.45752300 & Sum of electronic and thermal Enthalpies= & -1610.253228 \\
\hline 0 & 5.13284400 & 2.37838000 & -0.41546700 & Sum of electronic and thermal Free Energies= & -1610.357689 \\
\hline C & 3.77733800 & 0.21766800 & 0.75921500 & & \\
\hline C & 4.08248700 & -0.60742600 & -0.51645500 & & \\
\hline C & 2.63788100 & 1.18753600 & 0.57411900 & & \\
\hline C & 5.22136300 & -1.60686700 & -0.28614000 & & \\
\hline C & 1.31805900 & 0.70260800 & 0.85950800 & & \\
\hline C & 2.78133200 & 2.50272800 & 0.11820900 & & \\
\hline C & -1.99305300 & -2.21034200 & 0.39714600 & & \\
\hline C & -6.25121600 & -0.61967000 & -0.55350300 & & \\
\hline C & -1.11635900 & -1.18942000 & 0.84717900 & & \\
\hline C & -3.45488100 & -1.95438800 & 0.15760500 & & \\
\hline C & 5.55548100 & -2.43534700 & -1.53274000 & & \\
\hline C & -5.06774300 & -0.80778000 & -1.53288800 & & \\
\hline C & 0.18063800 & 1.55529700 & 0.81665800 & & \\
\hline C & 0.25078900 & -1.49636200 & 0.90368400 & & \\
\hline C & -6.22607900 & 0.69134200 & 0.25112300 & & \\
\hline C & -3.71557500 & -0.81232400 & -0.85335000 & & \\
\hline C & 1.60545900 & 3.33104200 & 0.00783800 & & \\
\hline C & 0.33593600 & 2.83936100 & 0.36417400 & & \\
\hline C & -6.24256300 & 1.98663100 & -0.57791100 & & \\
\hline C & -1.48435700 & -3.46651800 & 0.09403500 & & \\
\hline C & -1.69450300 & 0.03275700 & 1.44944100 & & \\
\hline C & 0.75431000 & -2.77316900 & 0.66120200 & & \\
\hline C & 6.68839500 & -3.43846400 & -1.29766100 & & \\
\hline C & -0.12431000 & -3.77355100 & 0.26520200 & & \\
\hline C & 4.13139100 & 3.02004800 & -0.26627500 & & \\
\hline C & -7.48329700 & 2.16043600 & -1.46004200 & & \\
\hline $\mathrm{H}$ & 4.67553900 & 0.75951700 & 1.04417500 & & \\
\hline $\mathrm{H}$ & 3.52385400 & -0.46344400 & 1.57212800 & & \\
\hline $\mathrm{H}$ & 4.35353900 & 0.07542500 & -1.32484500 & & \\
\hline $\mathrm{H}$ & 3.17991800 & -1.14006500 & -0.83726500 & & \\
\hline $\mathrm{H}$ & 6.11635800 & -1.06296900 & 0.03723600 & & \\
\hline $\mathrm{H}$ & 4.95904500 & -2.28063100 & 0.54036700 & & \\
\hline $\mathrm{H}$ & -6.29112500 & -1.46566900 & 0.14057400 & & \\
\hline $\mathrm{H}$ & -7.16944800 & -0.68087400 & -1.14399800 & & \\
\hline $\mathrm{H}$ & -3.94487000 & -2.86588300 & -0.18742600 & & \\
\hline $\mathrm{H}$ & -3.95335200 & -1.65187800 & 1.08281900 & & \\
\hline $\mathrm{H}$ & -5.18978100 & -1.76331400 & -2.05593800 & & \\
\hline $\mathrm{H}$ & -5.04941300 & -0.01495700 & -2.28151900 & & \\
\hline $\mathrm{H}$ & 5.82921400 & -1.75918300 & -2.35042200 & & \\
\hline $\mathrm{H}$ & 4.65675900 & -2.96824900 & -1.86786900 & & \\
\hline $\mathrm{H}$ & -5.34715300 & 0.70472100 & 0.90650500 & & \\
\hline $\mathrm{H}$ & -7.09507800 & 0.68729900 & 0.91868400 & & \\
\hline $\mathrm{H}$ & -0.51396700 & 3.50729800 & 0.33137600 & & \\
\hline $\mathrm{H}$ & -6.18068200 & 2.82860500 & 0.11928700 & & \\
\hline $\mathrm{H}$ & -5.33811800 & 2.04709800 & -1.19367000 & & \\
\hline $\mathrm{H}$ & -2.13732200 & -4.25079300 & -0.26802500 & & \\
\hline $\mathrm{H}$ & 1.81942400 & -2.94401800 & 0.75323800 & & \\
\hline $\mathrm{H}$ & 6.43305000 & -4.14944800 & -0.50589900 & & \\
\hline
\end{tabular}




\begin{tabular}{|lrrr|r|}
\hline$H$ & 6.90208400 & -4.01186000 & -2.20260400 & \\
$H$ & 7.61082800 & -2.93110200 & -1.00143400 & \\
$H$ & -7.54915300 & 1.39445400 & -2.23810300 & \\
$H$ & -8.40069200 & 2.10717400 & -0.86579700 & \\
$H$ & -7.47179900 & 3.13060600 & -1.96265300 & \\
$H$ & 2.52562400 & 4.88752600 & -0.57021700 & \\
$H$ & 1.19212000 & -5.16546800 & 0.14755600 & \\
$H$ & 5.02643800 & 4.64478000 & -0.72882200 & \\
\hline
\end{tabular}


Table S4: Cartesian coordinates of the TS of the selective compounds and $\mathrm{CH}_{3} \mathrm{OO}$ following the HAT mechanism optimized at $M 05-2 X / 6-311++G(d, p)$ level of theory in the gas phase

\begin{tabular}{|c|c|c|c|c|c|}
\hline Con & & & & 1 & \\
\hline Car & Coordinates & & & Frequency and Energy & \\
\hline 0 & 1.19888300 & 0.03067700 & 1.27914100 & Zero-point correction= & (Hartree/Particle) \\
\hline $\mathrm{O}$ & 1.01690200 & 4.28232100 & -0.08888500 & Thermal correction to Energy= & 5512 \\
\hline 0 & -1.21664700 & -1.12919900 & 0.75998100 & Thermal correction to Enthalpy= & 7456 \\
\hline $\mathrm{O}$ & 1.98764100 & 3.18853300 & 1.73000300 & Thermal correction to Gibbs Free Energy= & 0.270094 \\
\hline $\mathrm{O}$ & -2.81100700 & 2.80805100 & -1.34761900 & Sum of electronic and zero-point Energies= & -1635.543590 \\
\hline $\mathrm{O}$ & -3.01455600 & -1.19216700 & -1.31603400 & Sum of electronic and thermal Energies= & -1635.516027 \\
\hline $\mathrm{O}$ & -0.86913100 & 4.99494300 & -1.11561900 & Sum of electronic and thermal Enthalpies= & -1635.515083 \\
\hline $\mathrm{O}$ & -0.88329800 & -3.29274400 & 0.69394800 & Sum of electronic and thermal Free Energies= & -1635.602444 \\
\hline 0 & 5.00258100 & -1.83180100 & -0.87729700 & & \\
\hline 0 & 5.12764200 & 0.30708800 & 0.59316500 & & \\
\hline C & 0.39127500 & 2.06797400 & 0.26229600 & & \\
\hline C & 0.22823700 & 0.74987200 & 0.61194200 & & \\
\hline $\mathrm{C}$ & -0.64011900 & 2.73576100 & -0.36616300 & & \\
\hline $\mathrm{C}$ & 1.53985200 & 3.03578000 & 0.43673900 & & \\
\hline $\mathrm{C}$ & -0.97838600 & 0.13031600 & 0.28900700 & & \\
\hline $\mathrm{C}$ & -2.00862300 & 0.77446900 & -0.40655400 & & \\
\hline C & -1.83956500 & 2.13475700 & -0.71640300 & & \\
\hline C & 1.75034600 & -0.96121300 & 0.49182600 & & \\
\hline C & -0.23302700 & 4.12404100 & -0.58973500 & & \\
\hline $\mathrm{C}$ & 0.96648500 & -1.99421000 & 0.00007900 & & \\
\hline C & -3.24324000 & 0.07056400 & -0.80000500 & & \\
\hline $\mathrm{C}$ & 1.57561100 & -2.96843900 & -0.83416900 & & \\
\hline $\mathrm{C}$ & 3.12200900 & -0.86860500 & 0.24070400 & & \\
\hline C & -0.41792600 & -2.21429300 & 0.48467700 & & \\
\hline $\mathrm{C}$ & 3.70985500 & -1.85100000 & -0.58418700 & & \\
\hline $\mathrm{C}$ & 2.92065500 & -2.86712600 & -1.12644300 & & \\
\hline $\mathrm{C}$ & 0.79131200 & -4.10605600 & -1.42462000 & & \\
\hline$C$ & 3.93159200 & 0.20115000 & 0.81661400 & & \\
\hline $\mathrm{H}$ & 2.40661700 & 2.78103800 & -0.16772800 & & \\
\hline $\mathrm{H}$ & -3.98617800 & -0.12364800 & 0.24188300 & & \\
\hline $\mathrm{H}$ & -3.90626000 & 0.68574700 & -1.40572600 & & \\
\hline $\mathrm{H}$ & 3.39984200 & -3.58771600 & -1.77360800 & & \\
\hline $\mathrm{H}$ & 1.24100300 & 3.33738600 & 2.31829800 & & \\
\hline $\mathrm{H}$ & -0.17100800 & -3.76785600 & -1.80491100 & & \\
\hline $\mathrm{H}$ & 1.35794500 & -4.56090400 & -2.23342300 & & \\
\hline $\mathrm{H}$ & 0.58686400 & -4.85618800 & -0.66232000 & & \\
\hline $\mathrm{H}$ & -2.52271300 & 3.72344700 & -1.49260600 & & \\
\hline $\mathrm{H}$ & 3.42593900 & 0.91891900 & 1.47082600 & & \\
\hline $\mathrm{H}$ & -3.82772300 & -1.70662100 & -1.21337100 & & \\
\hline $\mathrm{H}$ & 5.40873500 & -1.05819800 & -0.42882900 & & \\
\hline 0 & -4.89408800 & -0.63646400 & 0.94045900 & & \\
\hline $\mathrm{O}$ & -4.99084300 & -1.91908400 & 0.46334300 & & \\
\hline$C$ & -4.27996900 & -2.79606400 & 1.34765600 & & \\
\hline $\mathrm{H}$ & -4.71586000 & -2.72417100 & 2.34152500 & & \\
\hline $\mathrm{H}$ & -4.42307400 & -3.79250000 & 0.93645100 & & \\
\hline $\mathrm{H}$ & -3.22499300 & -2.53604800 & 1.35728400 & & \\
\hline Con & & & & 2 & \\
\hline Car & Coordinates & & & Frequency and Energy & \\
\hline 0 & 0.47435600 & -0.05941100 & -1.46126400 & Zero-point correction= & (Hartree/Particle) \\
\hline $\mathrm{O}$ & -3.28285900 & -2.17718100 & -0.29159700 & Thermal correction to Energy= & 9569 \\
\hline
\end{tabular}




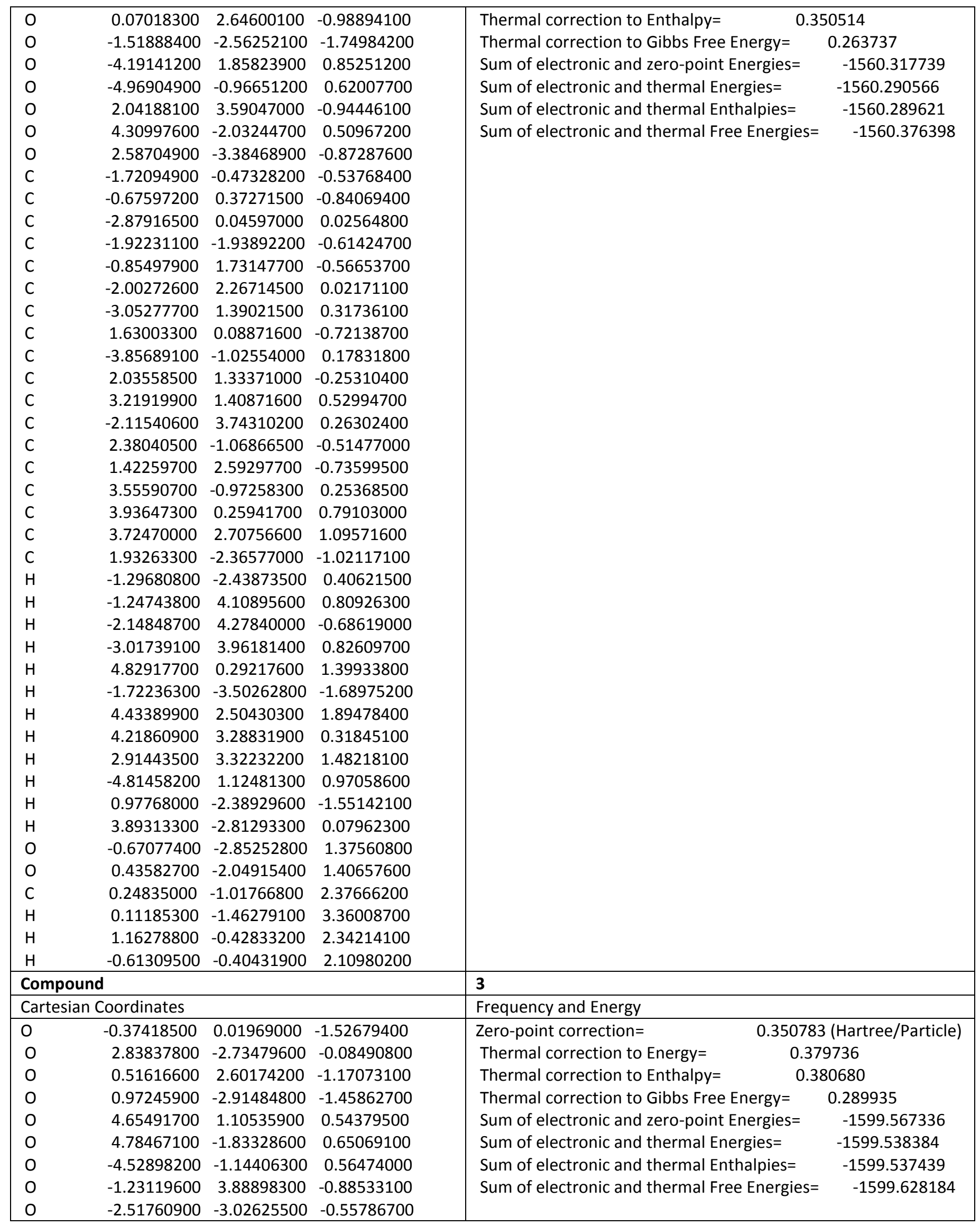




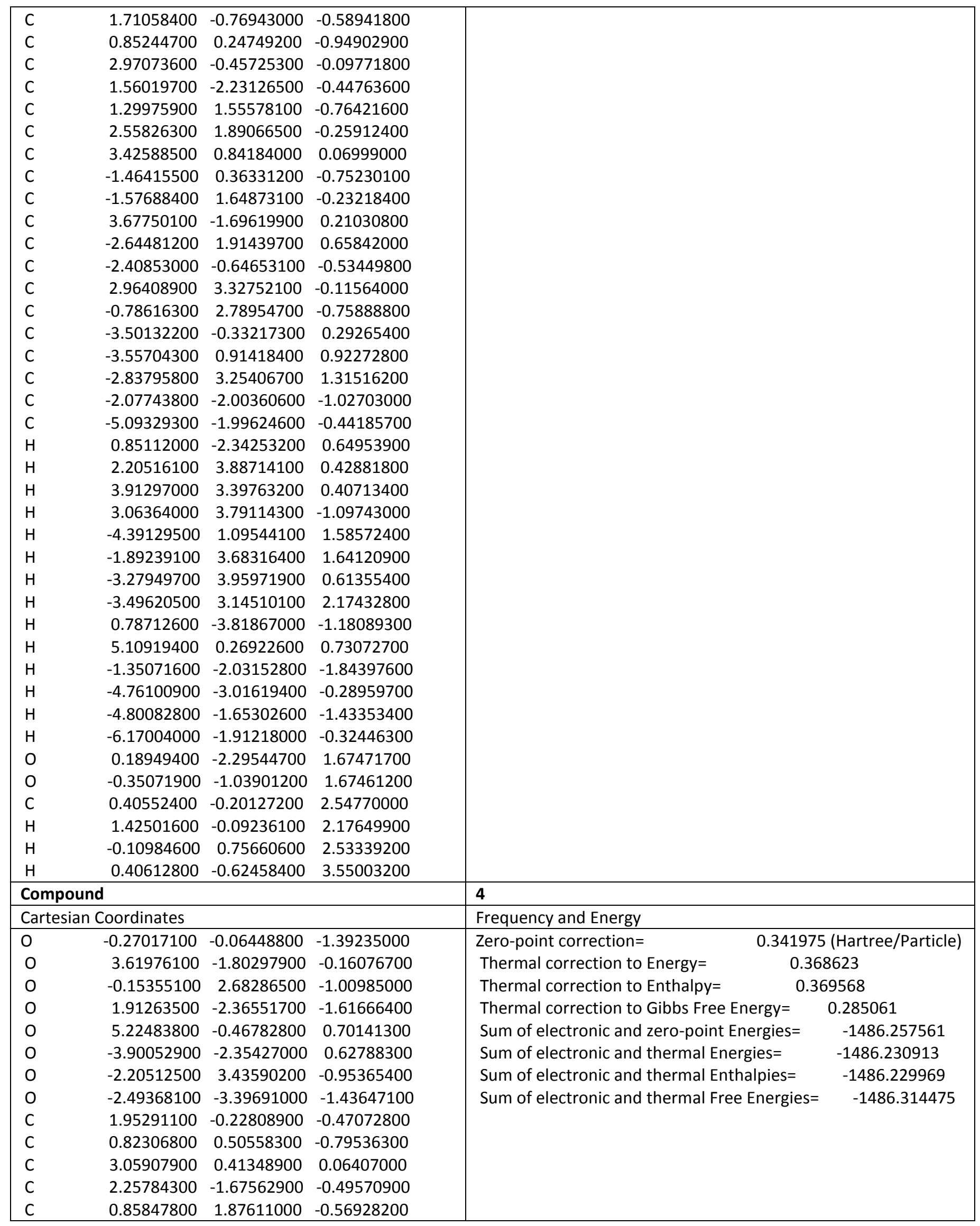




\begin{tabular}{|c|c|c|c|c|}
\hline $\mathrm{C}$ & 1.96632800 & 2.52851400 & -0.00610300 & \\
\hline $\mathrm{C}$ & 3.08972900 & 1.77427500 & 0.31091700 & \\
\hline C & -1.44742700 & -0.01697600 & -0.65740100 & \\
\hline $\mathrm{C}$ & -1.97271300 & 1.20337800 & -0.21568000 & \\
\hline $\mathrm{C}$ & 4.12581000 & -0.58882600 & 0.26759900 & \\
\hline C & -3.14291100 & 1.18575200 & 0.57629700 & \\
\hline C & -2.06810800 & -1.23169200 & -0.42373900 & \\
\hline C & 1.91846800 & 4.01588800 & 0.18843100 & \\
\hline C & -1.49350300 & 2.50366500 & -0.73386000 & \\
\hline $\mathrm{C}$ & -3.24089300 & -1.21894500 & 0.34046400 & \\
\hline $\mathrm{C}$ & -3.74006500 & -0.02854700 & 0.86171300 & \\
\hline $\mathrm{C}$ & -3.77826200 & 2.43902800 & 1.11832300 & \\
\hline $\mathrm{C}$ & -1.48288900 & -2.54477300 & -0.87074200 & \\
\hline $\mathrm{H}$ & 1.64529300 & -2.16123000 & 0.53123700 & \\
\hline $\mathrm{H}$ & 2.83618300 & 4.36511100 & 0.65543500 & \\
\hline $\mathrm{H}$ & 1.78976200 & 4.52045000 & -0.76890500 & \\
\hline $\mathrm{H}$ & 1.07092200 & 4.29764700 & 0.81263400 & \\
\hline $\mathrm{H}$ & -4.63090600 & -0.07434600 & 1.47234000 & \\
\hline $\mathrm{H}$ & -3.03518900 & 3.15047500 & 1.47243600 & \\
\hline $\mathrm{H}$ & -4.35112900 & 2.94139100 & 0.34076700 & \\
\hline $\mathrm{H}$ & -4.44386300 & 2.18037500 & 1.93919700 & \\
\hline $\mathrm{H}$ & 2.25700500 & -3.26265100 & -1.55030200 & \\
\hline $\mathrm{H}$ & -1.10250900 & -3.08695000 & -0.00648100 & \\
\hline $\mathrm{O}$ & 1.02533500 & -2.50404500 & 1.55037200 & \\
\hline $\mathrm{O}$ & -0.00705100 & -1.60793400 & 1.62145700 & \\
\hline C & 0.30432100 & -0.58899500 & 2.57158400 & \\
\hline $\mathrm{H}$ & 1.19158100 & -0.03895500 & 2.25675300 & \\
\hline $\mathrm{H}$ & -0.56766500 & 0.06192200 & 2.57578300 & \\
\hline $\mathrm{H}$ & 0.45885300 & -1.03713000 & 3.55105400 & \\
\hline $\mathrm{H}$ & 3.97445600 & 2.23692600 & 0.72651100 & \\
\hline $\mathrm{H}$ & -3.60231600 & -3.05359500 & 0.02306700 & \\
\hline $\mathrm{H}$ & -2.77718800 & -3.02684600 & -2.27559400 & \\
\hline $\mathrm{H}$ & -0.66857000 & -2.39021000 & -1.57161600 & \\
\hline \multicolumn{4}{|c|}{ Compound } & 5 \\
\hline \multicolumn{4}{|c|}{ Cartesian Coordinates } & Frequency and Energy \\
\hline 0 & 0.21788800 & 0.17954800 & -1.38030300 & 0.375962 (Hartree/Particle) \\
\hline 0 & 2.14262400 & -3.43425800 & 0.43003600 & Thermal correction to Energy= \\
\hline 0 & 1.98299200 & 2.28332100 & -1.14677900 & Thermal correction to Enthalpy= \\
\hline 0 & 0.74127900 & -3.08623900 & -1.40304600 & Thermal correction to Gibbs Free Energy $=\quad 0.315088$ \\
\hline 0 & 5.16438500 & -0.45009400 & 1.03536400 & Sum of electronic and zero-point Energies= \\
\hline 0 & 4.21687600 & -3.21165900 & 1.30268600 & Sum of electronic and thermal Energies= \\
\hline $\mathrm{O}$ & -3.60827200 & 0.30616000 & 1.33007000 & Sum of electronic and thermal Enthalpies= \\
\hline $\mathrm{O}$ & 0.70724900 & 4.04435300 & -1.41678800 & Sum of electronic and thermal Free Energies $=\quad-1600.832213$ \\
\hline $\mathrm{C}$ & 1.82374500 & -1.23003500 & -0.24723200 & \\
\hline $\mathrm{C}$ & 1.43144600 & -0.01590000 & -0.75449100 & \\
\hline $\mathrm{C}$ & 3.06986900 & -1.34989600 & 0.33345400 & \\
\hline $\mathrm{C}$ & 1.14107600 & -2.57792800 & -0.18504000 & \\
\hline $\mathrm{C}$ & 2.30688100 & 1.06338200 & -0.61193600 & \\
\hline $\mathrm{C}$ & 3.56761200 & 0.96278700 & -0.02152300 & \\
\hline $\mathrm{C}$ & 3.95912900 & -0.29141400 & 0.46079200 & \\
\hline $\mathrm{C}$ & -0.63376400 & 0.90076300 & -0.56101100 & \\
\hline $\mathrm{C}$ & -0.34801000 & 2.24282800 & -0.30597700 & \\
\hline $\mathrm{C}$ & 3.25605800 & -2.73766400 & 0.75825800 & \\
\hline $\mathrm{C}$ & -1.21526500 & 2.97890100 & 0.51650500 & \\
\hline
\end{tabular}




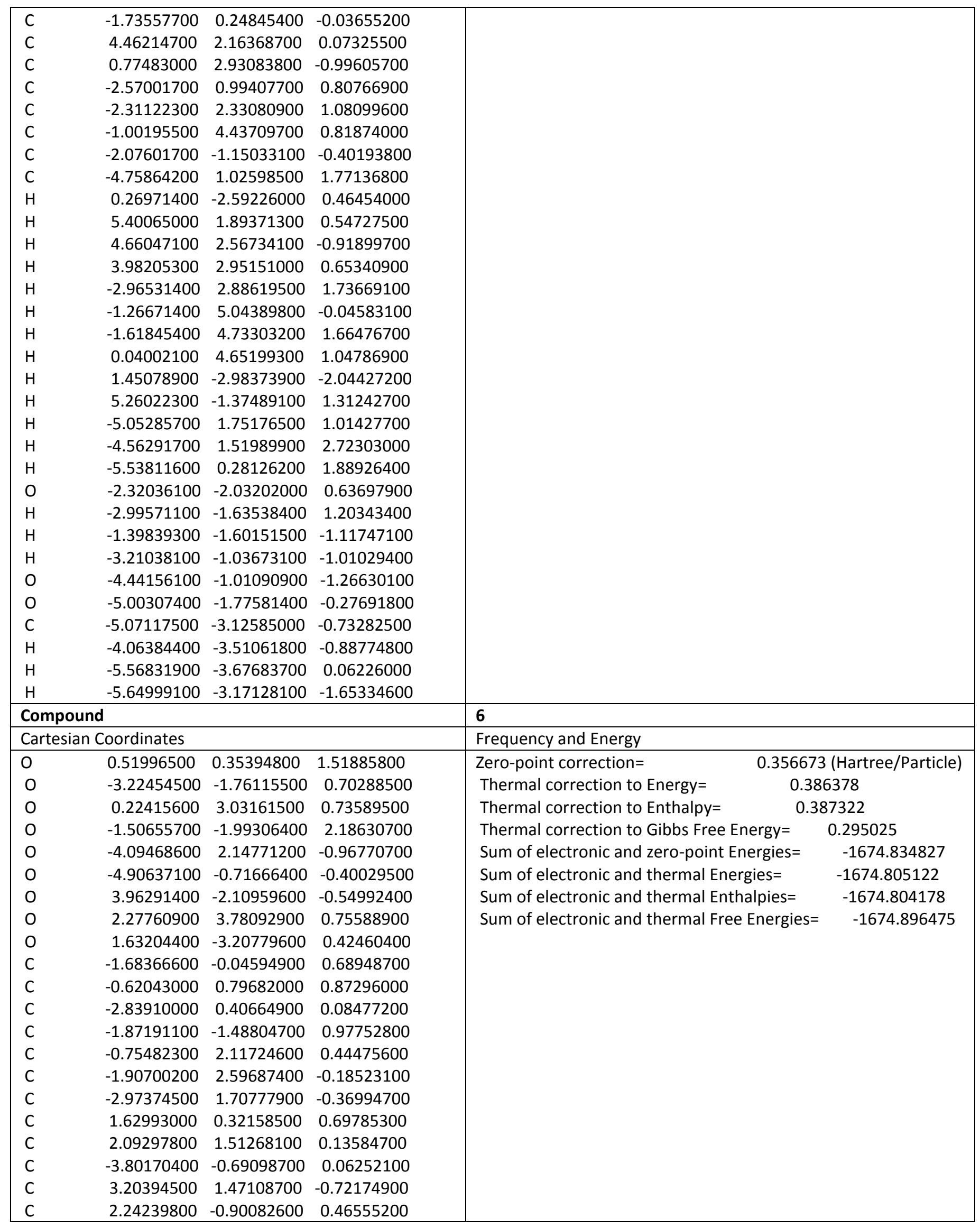




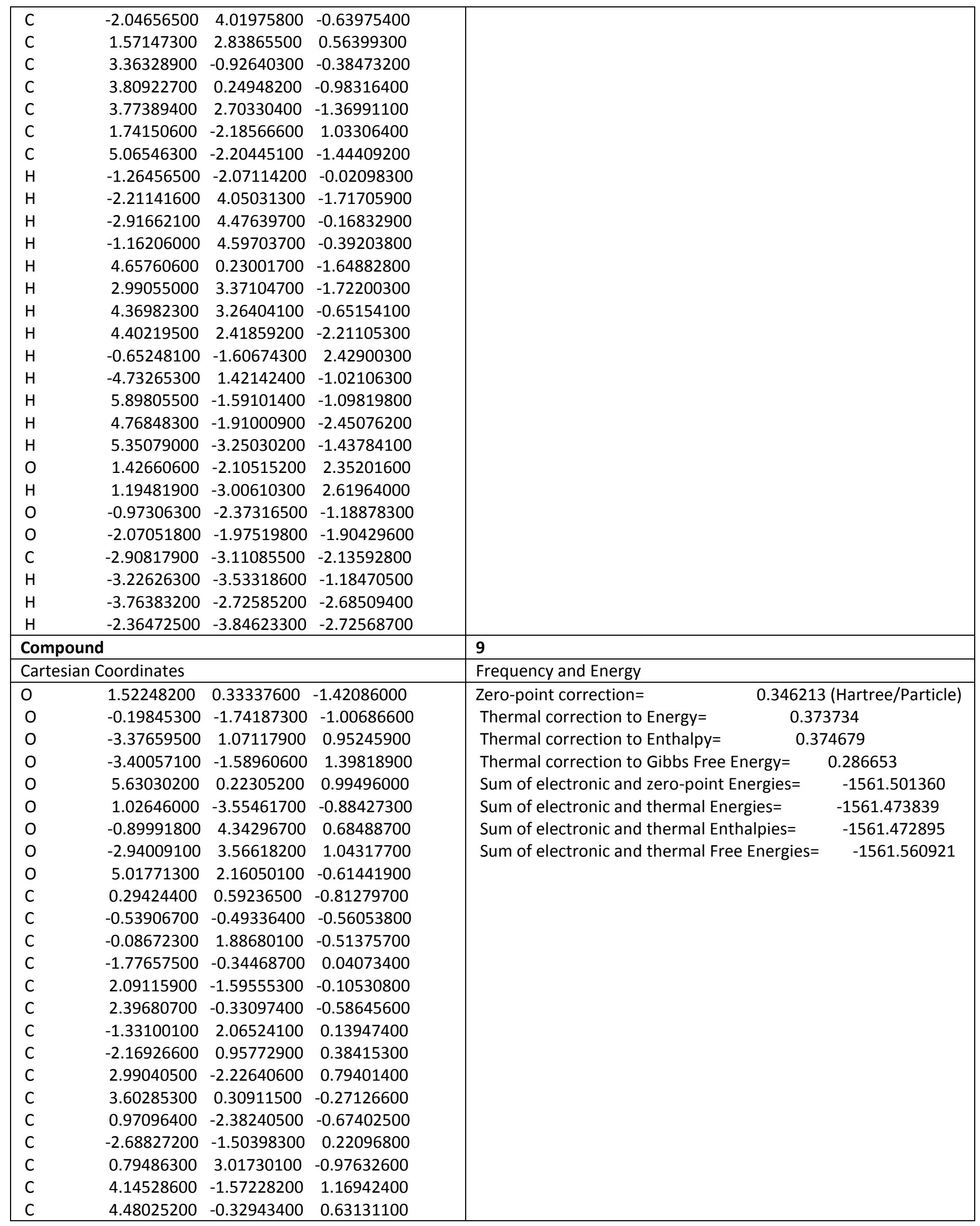




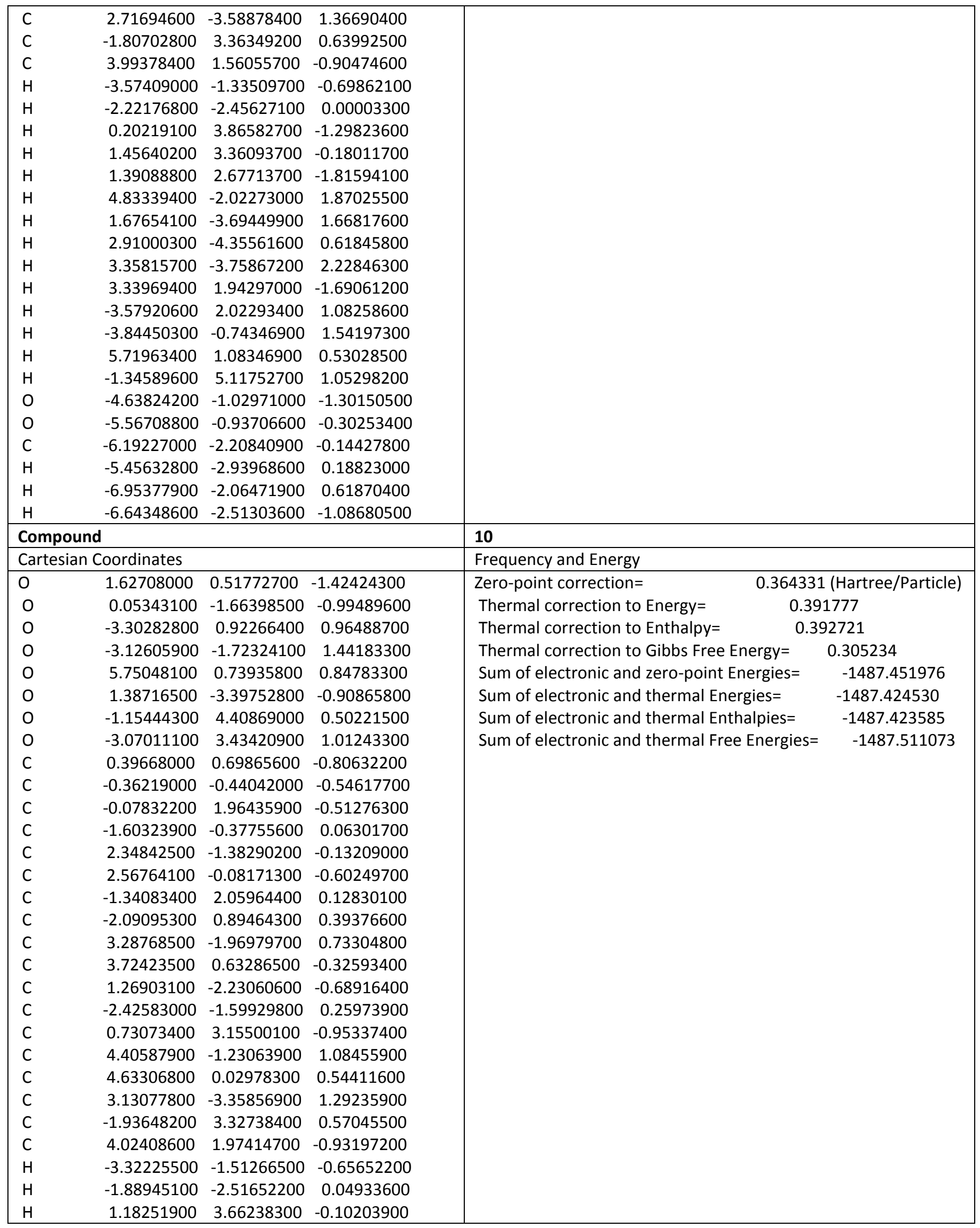




\begin{tabular}{|rrrr|r|}
\hline$H$ & 1.51020600 & 2.82642100 & -1.62875100 & \\
$H$ & 0.10050700 & 3.87718100 & -1.46433600 & \\
$H$ & 5.12903100 & -1.65505000 & 1.77099600 & \\
$H$ & 2.10790600 & -3.55124800 & 1.60922700 & \\
$H$ & 3.37458300 & -4.10222000 & 0.53569800 & \\
$H$ & 3.79411100 & -3.48622400 & 2.14560600 & \\
$H$ & 3.55548200 & 2.06095300 & -1.90808700 & \\
$H$ & -3.56960800 & 1.86008300 & 1.09237500 & \\
$H$ & -3.62401700 & -0.90668100 & 1.57919500 & \\
$H$ & 6.31943000 & 0.22393000 & 1.42351700 & \\
$H$ & -1.68129200 & 5.14772100 & 0.83424000 & \\
$H$ & 5.09895400 & 2.09306000 & -1.04163200 & \\
$H$ & 3.66368100 & 2.78567400 & -0.29859400 & \\
O & -4.41175100 & -1.31190900 & -1.26707600 & \\
O & -5.34698300 & -1.28270700 & -0.26981900 & \\
C & -5.85397100 & -2.60151100 & -0.08475100 & \\
$H$ & -5.05552000 & -3.25551900 & 0.26412900 & \\
$H$ & -6.62753500 & -2.51183000 & 0.67447500 & \\
$H$ & -6.27311900 & -2.96647800 & -1.02078300 & \\
\hline
\end{tabular}


Table S5. The calculated rate constants $\left(k_{\mathrm{app}}, \mathrm{M}^{-1} \mathrm{~s}^{-1}, 298.15 \mathrm{~K}\right)$ of the reaction between the depsidones and the radicals in water following SET mechanism at the M05-2X/6-31+G(d) method.

\begin{tabular}{|c|c|c|c|c|c|}
\hline \multirow{2}{*}{ Comp. } & \multirow{2}{*}{$\begin{array}{l}\text { Main } \\
\text { forms }\end{array}$} & \multirow{2}{*}{$\%$} & \multicolumn{3}{|c|}{$k_{\text {app }}$} \\
\hline & & & $\mathrm{HO}^{\circ}$ & $\mathrm{HOO}^{\circ}$ & $\mathrm{CH}_{3} \mathrm{OO} \cdot$ \\
\hline 1 & $\mathrm{HA}^{-}$ & $>90$ & $9.80 \times 10^{5}$ & $4.80 \times 10^{-11}$ & $1.90 \times 10^{-12}$ \\
\hline 2 & $\mathrm{HA}^{-}$ & $>90$ & $1.00 \times 10^{7}$ & $9.70 \times 10^{-10}$ & $4.20 \times 10^{-11}$ \\
\hline 3 & $\mathrm{HA}^{-}$ & $>90$ & $1.60 \times 10^{5}$ & $2.60 \times 10^{-13}$ & $5.40 \times 10^{-15}$ \\
\hline 4 & $\mathrm{HA}^{-}$ & $>90$ & $8.50 \times 10^{9}$ & 4.6 & $2.60 \times 10^{-1}$ \\
\hline 5 & $\mathrm{HA}^{-}$ & $>90$ & $2.70 \times 10^{7}$ & $2.90 \times 10^{-9}$ & $1.20 \times 10^{-10}$ \\
\hline 6 & $\mathrm{HA}^{-}$ & $\sim 100$ & $2.30 \times 10^{8}$ & $2.50 \times 10^{-7}$ & $1.50 \times 10^{-8}$ \\
\hline \multirow{2}{*}{7} & $\mathrm{H}_{2} \mathrm{~A}$ & 76 & $5.40 \times 10^{-14}$ & $7.10 \times 10^{-32}$ & $9.50 \times 10^{-35}$ \\
\hline & $\mathrm{HA}^{-}$ & 24 & $8.20 \times 10^{9}$ & $4.70 \times 10^{-2}$ & $1.20 \times 10^{-3}$ \\
\hline \multirow{2}{*}{8} & $\mathrm{H}_{2} \mathrm{~A}^{-}$ & 73 & $5.90 \times 10^{9}$ & $3.10 \times 10^{-4}$ & $2.30 \times 10^{-5}$ \\
\hline & $\mathrm{HA}^{2-}$ & 27 & $8.50 \times 10^{9}$ & $3.90 \times 10^{-4}$ & $1.80 \times 10^{-6}$ \\
\hline \multirow{2}{*}{9} & $\mathrm{H}_{2} \mathrm{~A}^{-}$ & 73 & $4.00 \times 10^{8}$ & $6.60 \times 10^{-7}$ & $4.00 \times 10^{-8}$ \\
\hline & $\mathrm{HA}^{2-}$ & 27 & $2.30 \times 10^{9}$ & $1.70 \times 10^{-5}$ & $1.10 \times 10^{-6}$ \\
\hline \multirow{2}{*}{10} & $\mathrm{H}_{2} \mathrm{~A}^{-}$ & 73 & $1.70 \times 10^{9}$ & $8.40 \times 10^{-6}$ & $5.50 \times 10^{-7}$ \\
\hline & $\mathrm{HA}^{2-}$ & 27 & $8.40 \times 10^{9}$ & $6.00 \times 10^{-8}$ & $4.40 \times 10^{-11}$ \\
\hline 11 & $A^{-}$ & $\sim 100$ & $8.50 \times 10^{9}$ & $6.70 \times 10^{-5}$ & $1.30 \times 10^{-6}$ \\
\hline \multirow{2}{*}{12} & $\mathrm{H}_{2} \mathrm{~A}^{-}$ & 73 & $3.10 \times 10^{9}$ & $9.00 \times 10^{-5}$ & $7.10 \times 10^{-6}$ \\
\hline & $\mathrm{HA}^{2-}$ & 27 & $4.40 \times 10^{9}$ & $7.80 \times 10^{-5}$ & $5.50 \times 10^{-6}$ \\
\hline
\end{tabular}


Table S6. The method to compute rate constant following the transition state theory and the quantum mechanics based test for overall free radical scavenging activity (QM-ORSA) protocol.

The rate constant was computed according to the transition state theory (at $298.15 \mathrm{~K}, 1 \mathrm{M}$ standard state) and following the quantum mechanics based test for overall free radical scavenging activity (QM-ORSA) protocol ${ }^{1}$

$$
k=\sigma \kappa \frac{k_{B} T}{h} e^{-\left(\Delta G^{\ddagger}\right) / R T}
$$

Where $k_{B}$ and $h$ are the Boltzmann and Planck constants, respectively, $\Delta G^{\neq}$is Gibbs free energy of activation of the studied reaction, $\sigma$ is the reaction symmetry number that represents reaction path degeneracy (which was calculated following the literature ${ }^{2,3}$ ), the number of possible different but equivalent reaction pathways, and $\kappa$ accounts for tunneling corrections which were calculated using Eckart barrier ${ }^{4}$. The Marcus Theory was used to estimate the reaction barriers of SET reactions ${ }^{5-8}$. To avoid over-penalizing entropy losses in solution, in this study the solvent cage effects were included following the corrections proposed by Okuno ${ }^{9}$, adjusted with the free volume theory according to the Benson correction ${ }^{1,10}$. These corrections have been successfully used to study the radical scavenging activity of antioxidants in solution ${ }^{1,11-13}$ and are in good agreement with activity data independently obtained by Ardura et a ${ }^{14}$. For rate constants that were close to the diffusion limit a correction was applied to yield realistic results ${ }^{1}$. The apparent rate constants $\left(k_{\mathrm{app}}\right)$ were calculated following the Collins-Kimball theory in the solvents at $298.15 \mathrm{~K}^{15}$; the steady-state Smoluchowski rate constant $\left(k_{D}\right)$ for an irreversible bimolecular diffusion-controlled reaction was calculated following the literature ${ }^{1,16}$ 


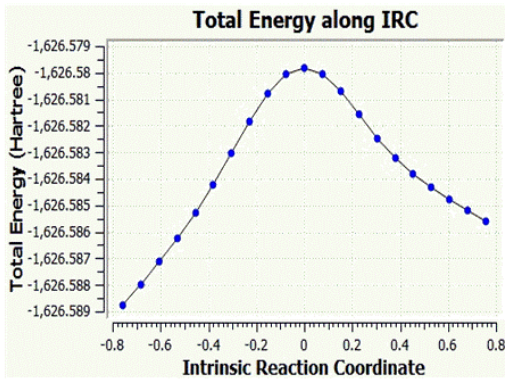

1-C5'-H-OOCH3

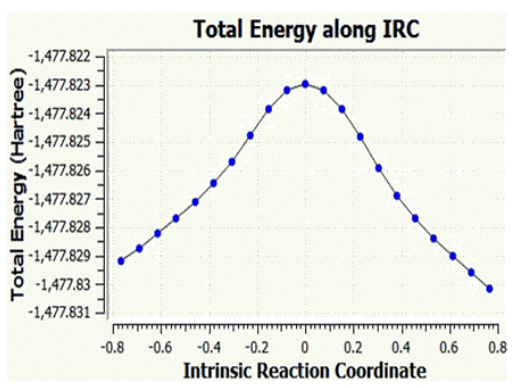

4-C1-H-OOCH3

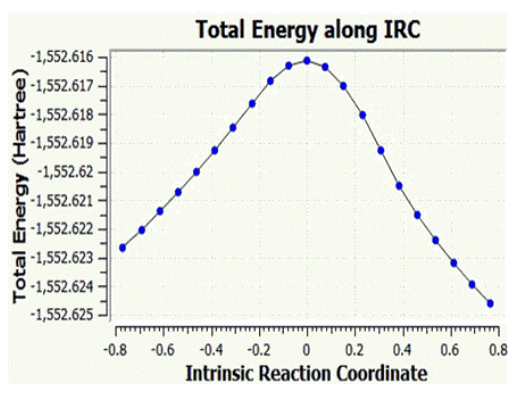

9-C9'-H-OOCH3

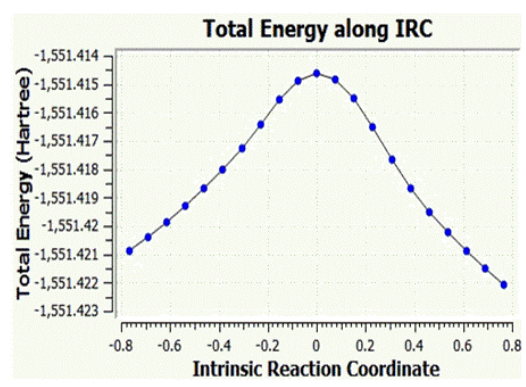

2-C1-H-OOCH3

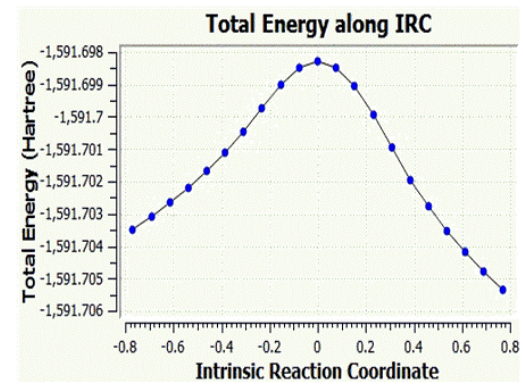

5-C11'-H-OOCH3

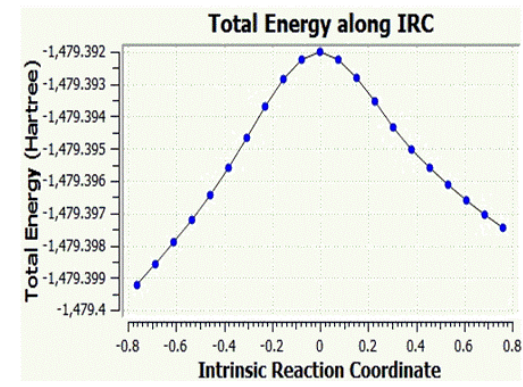

10-C9'-H-OOCH3

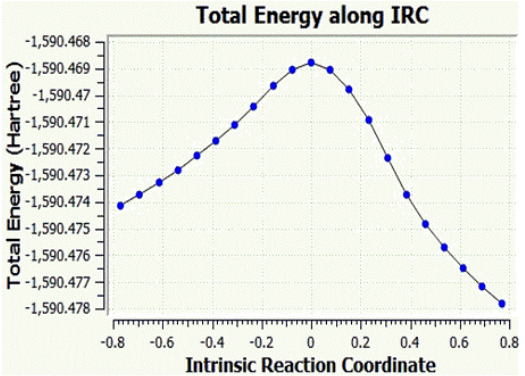

3-C1-H-OOCH3

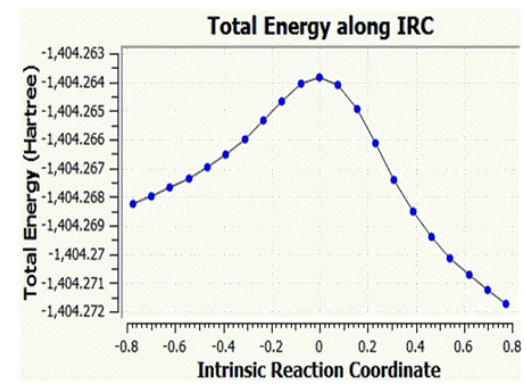

6-C1-H-OOCH3

Figure S1: IRC plots for all transition states related to the reaction of $\mathrm{CH}_{3} \mathrm{OO} \cdot$ radical with the selective depsidones 
Table S7. The lowest calculated BDEs, IEs and PAs of the depsidones by the (RO)B3LYP-GD3/6$311++G(2 d f, 2 p) / / B 3 L Y P-G D 3 / 6-311 G(d, p)$ level and error bars compared with the the (RO)B3LYP/6$311++G(2 d f, 2 p) / / B 3 L Y P / 6-311 G(d, p)$ level in the gas phase.

\begin{tabular}{|c|c|c|c|c|c|c|}
\hline Comp. & BDE & $\begin{array}{c}\Delta B D E \\
\text { (B3LYP-GD3)-B3LYP) }\end{array}$ & PA & $\begin{array}{c}\Delta P A \\
\text { (B3LYP-GD3) } \\
\text { B3LYP) }\end{array}$ & IE & $\begin{array}{c}\Delta \mathrm{IE} \\
\text { (B3LYP-GD3) } \\
\text { B3LYP) }\end{array}$ \\
\hline $\mathbf{1}$ & 78.1 & 1.2 & 310.2 & 0.1 & 192.1 & 0.4 \\
\hline $\mathbf{2}$ & 81.3 & 1.4 & 312.3 & 0.1 & 189.8 & 0.7 \\
\hline $\mathbf{3}$ & 81.9 & 1.5 & 315.1 & 0.5 & 188.4 & 1.1 \\
\hline $\mathbf{4}$ & 81.6 & 1.7 & 316.1 & -0.3 & 191.4 & 0.8 \\
\hline $\mathbf{5}$ & 75.3 & 0.9 & 320.4 & 0.3 & 182.6 & 1.1 \\
\hline $\mathbf{6}$ & 76.5 & 1.3 & 315.6 & -0.2 & 183.4 & 0.5 \\
\hline $\mathbf{7}$ & 84.3 & 0.7 & 325.1 & 0.4 & 186.6 & 0.4 \\
\hline $\mathbf{8}$ & 86.3 & 0.5 & 320.3 & 0.4 & 173.5 & 0.7 \\
\hline $\mathbf{9}$ & 82.8 & 0.4 & 310.7 & 0.2 & 183.9 & 0.8 \\
\hline $\mathbf{1 0}$ & $\mathbf{7 9 . 8}$ & 1.5 & 315.7 & 0.6 & 176.0 & 0.7 \\
\hline $\mathbf{1 1}$ & 88.3 & 0.6 & 321.3 & 0.7 & 176.1 & 0.8 \\
\hline $\mathbf{1 2}$ & 85.7 & 1.2 & 316.9 & 0.5 & 176.2 & 1.6 \\
\hline
\end{tabular}


Table S8. Calculated $\Delta \mathrm{G}^{\neq}(\mathrm{kcal} / \mathrm{mol}), \quad \kappa$, and $k_{\text {eck }}\left(\mathrm{M}^{-1} \mathrm{~s}^{-1}\right)$ at $298.15 \mathrm{~K}$ of the reaction between the selective depsidones and $\mathrm{CH}_{3} \mathrm{OO} 0^{\circ}$ in the gas phase by using the M05-2X-GD3/6-311++G(d,p) method.

\begin{tabular}{|c|c|c|c|c|c|}
\hline Comp. & $\Delta \mathbf{G}^{*}$ & $\Delta \mathbf{G}^{\neq}{ }_{(\mathrm{M} 05-2 \mathrm{X}-\mathrm{GD} 3)}-\Delta \mathrm{G}^{\neq}{ }_{(\mathrm{M} 05-2 \mathrm{X})}$ & $\kappa$ & $\mathbf{k}_{\text {eck }}$ & $\left.k_{(\mathrm{M} 05-2 \mathrm{X}-\mathrm{GD})}\right) \boldsymbol{k}_{(\mathrm{M} 05-2 \mathrm{X})}$ \\
\hline 1 & 19.8 & -0.8 & 82.3 & 1.63 & 1.7 \\
\hline 2 & 20.5 & -1.0 & 86.9 & 0.51 & 2.0 \\
\hline 3 & 20.8 & -1.7 & 91.3 & 0.32 & 6.7 \\
\hline 4 & 19.8 & -1.5 & 84.5 & 1.61 & 6.5 \\
\hline 5 & 19.7 & -0.5 & 79.5 & 1.79 & 1.3 \\
\hline 6 & 20.1 & -0.4 & 123.4 & 1.41 & 1.1 \\
\hline 9 & 20.6 & -0.8 & 58.3 & 0.27 & 1.6 \\
\hline 10 & 20.3 & -0.8 & 48.6 & 0.40 & 1.7 \\
\hline
\end{tabular}

\section{References}

(1) Galano, A.; Alvarez-Idaboy, J. R. A computational methodology for accurate predictions of rate constants in solution: Application to the assessment of primary antioxidant activity. J. Comput. Chem. 2013, 34, 2430-2445.

(2) Pollak, E.; Pechukas, P. Symmetry numbers, not statistical factors, should be used in absolute rate theory and in broensted relations. J. Am. Chem. Soc. 1978, 100, 2984-2991.

(3) Fernández-Ramos, A.; Ellingson, B. A.; Meana-Pañeda, R.; Marques, J. M.; Truhlar, D. G. Symmetry numbers and chemical reaction rates. Theor. Chem. Acc. 2007, 118, 813-826.

(4) Eckart, C. The penetration of a potential barrier by electrons. Phy. Rev. 1930, 35, 1303.

(5) Marcus, R. A. Chemical and electrochemical electron-transfer theory. Annu. Rev. Phys. Chem. 1964, 15, 155-196.

(6) Marcus, R. A. Electron transfer reactions in chemistry. Theory and experiment. Rev. Mod. Phys. 1993, 65, 599.

(7) Lu, Y.; Wang, A.; Shi, P.; Zhang, H. A theoretical study on the antioxidant activity of piceatannol and isorhapontigenin scavenging nitric oxide and nitrogen dioxide radicals. PloS one 2017, 12, e0169773.

(8) Lu, Y.; Wang, A.; Shi, P.; Zhang, H.; Li, Z. Quantum chemical study on the antioxidation mechanism of piceatannol and isorhapontigenin toward hydroxyl and hydroperoxyl radicals. PloS one 2015, 10, e0133259. 
(9) Okuno, Y. Theoretical investigation of the mechanism of the baeyer-villiger reaction in nonpolar solvents. Chem.: Eur. J. 1997, 3, 212-218.

(10) Benson, S., The foundations of chemical kinetics: . Malabar, Florida, 1982.

(11) luga, C.; Alvarez-Idaboy, J. R.; Vivier-Bunge, A. Ros initiated oxidation of dopamine under oxidative stress conditions in aqueous and lipidic environments. J. Phys. Chem. B 2011, 115, 12234-12246.

(12) Alvarez-Idaboy, J. R.; Reyes, L.; Mora-Diez, N. The mechanism of the baeyer-villiger rearrangement: Quantum chemistry and tst study supported by experimental kinetic data. Org. Biomol. Chem. 2007, 5, 3682-3689.

(13) Alvarez-Idaboy, J. R.; Reyes, L.; Cruz, J. A new specific mechanism for the acid catalysis of the addition step in the baeyer- villiger rearrangement. Org. Lett. 2006, 8, 1763-1765.

(14) Ardura, D.; López, R.; Sordo, T. L. Relative gibbs energies in solution through continuum models: Effect of the loss of translational degrees of freedom in bimolecular reactions on gibbs energy barriers. J. Phys. Chem. B 2005, 109, 23618-23623.

(15) Collins, F. C.; Kimball, G. E. Diffusion-controlled reaction rates. J. Colloid Sci. 1949, 4, 425437.

(16) Von Smoluchowski, M. Mathematical theory of the kinetics of the coagulation of colloidal solutions. Z. Phys. Chem 1917, 92, 129-68. 